\title{
GEOCHEMICAL DATA ON
}

CONCENTRATIONS OF INORGANIC

CONSTITUENTS AND POLYCHLORINATED

BIPHENYL CONGENERS IN STREAMBED

SEDIMENTS IN TRIBUTARIES TO LAKE

CHAMPLAIN IN NEW YORK, VERMONT,

AND QUEBEC, 1992

By JOHN A. COLMAN and STEWART F. CLARK

U.S. GEOLOGICAL SURVEY

Open-File Report 94-472

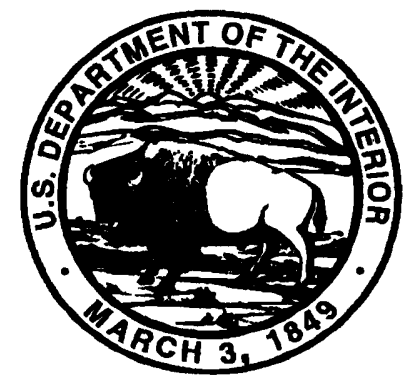

Bow, New Hampshire 


\title{
U.S. DEPARTMENT OF THE INTERIOR \\ BRUCE BABBITT, Secretary
}

\author{
U.S. GEOLOGICAL SURVEY \\ Gordon P. Eaton, Director
}

For additional information write to:

District Chief

U.S. Geological Survey

525 Cllnton Street

Bow, NH 03304
Copies of this report may be purchased from:

U.S. Geological Survey

Earth Sclence Information Center

Open-Flle Reports Section

Box 25286 MS 517

Denver Federal Center

Denver, CO 80225 


\section{CONTENTS}

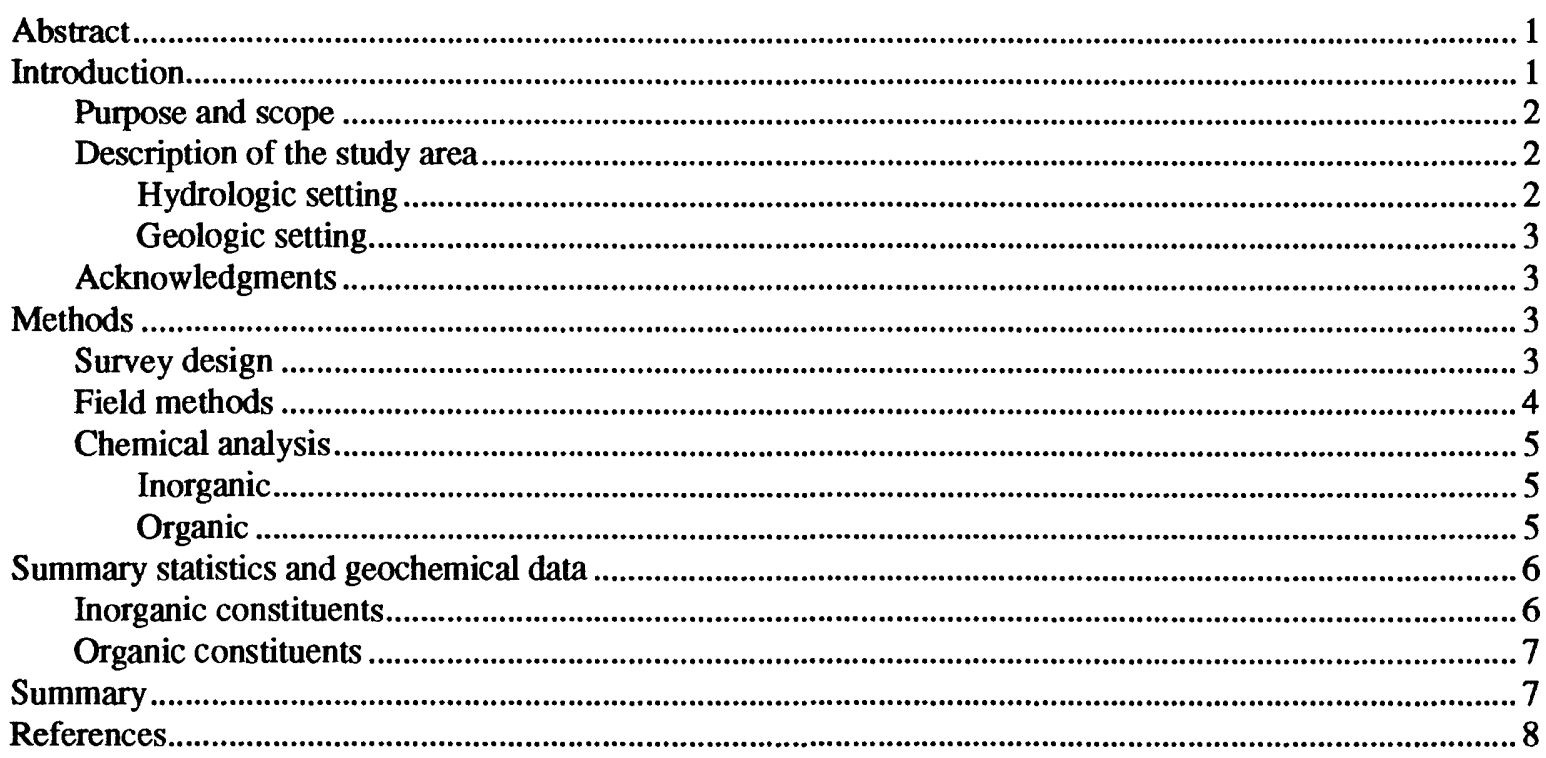

\section{Figures}

1. Map showing sample-site locations in the Lake Champlain Basin ......................................................48

2. Histograms of polychlorinated biphenyl congeners from stream- and lake-bed samples of

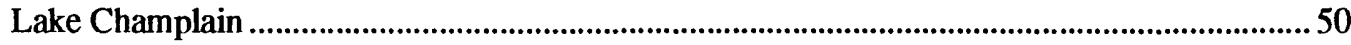

3. Histograms of polychlorinated biphenyl congeners in Aroclor mixtures ..............................................64

\section{Tables}

1. Location and sample-type data for sites samples in the Lake Champlain Basin.....................................9

2. Minimum reporting levels for the streambed sediments survey of the Lake Champlain Basin............. 12

3. Standard reference-material concentrations for inorganic-constituent analyses for tributaries

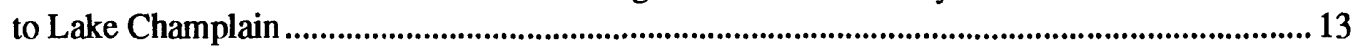

4. Results, in percent, from one-way, nested analysis of variance of ranked sediment-constituentconcentration data for tributaries to Lake Champlain ....................................................................... 14

5. Percentile distribution of element concentrations in 86 samples of fine-fraction streambed sediment for tributaries to Lake Champlain............................................................................. 15

6. Inorganic-constituent concentrations in fine-fraction streambed sediment for tributaries to Lake Champlain

7. Domain, International Union of Practical and Applied Chemistry (IUPAC) structure numberand name of polychlorinated biphenyl congeners in order of elution from the gas chromatograph

8. Concentrations of polychlorinated biphenyl congener standard-reference material HS-2 and

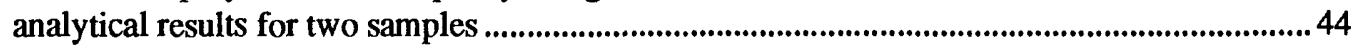

9. Carbonate, organic, total carbon concentrations, and polychlorinated biphenyl compound detections in streambed sediment for tributaries to Lake Champlain 


\section{CONVERSION FACTORS AND ABBREVIATED WATER-QUALTY UNITS}

\begin{tabular}{lcl}
\hline Multiply & By & To obtaln \\
\hline inch (in.) & 25.4 & millimeter \\
foot (ft) & 0.3048 & meter \\
mile (mi) & 1.609 & kilometer \\
square mile $\left(\mathrm{mi}^{2}\right.$ ) & 2.590 & square kilometer \\
gallon (gal) & 3.785 & liter \\
\hline
\end{tabular}

\section{Additional Conversions}

In this report certain units of measurement, by convention, use the metric system and include the following:

\begin{tabular}{lll}
\hline Mulitiply & By & To obtain \\
\hline centimeter $(\mathrm{cm})$ & 0.3937 & inch \\
millimeter $(\mathrm{mm})$ & 0.03937 & inch \\
micrometer $(\mu \mathrm{m})$ & 0.00003937 & inch \\
kilogram $(\mathrm{kg})$ & 2.205 & pound, avoirdupois \\
gram $(\mathrm{g})$ & 0.03527 & ounce, avoirdupois \\
\hline
\end{tabular}

In this report, the volume of chemical compounds or water is expressed in liters $(\mathrm{L})$, milliliters $(\mathrm{mL})$, and microliters $(\mu \mathrm{L})$. Weight of chemical compounds is expressed in grams ( $\mathrm{g}$ ) and nanograms (ng). Concentrations of polychlorinated biphenyl congeners in sediment are in micrograms per gram $(\mu \mathrm{g} / \mathrm{g})$. Concentrations of inorganic constituents are in micrograms per gram $(\mu \mathrm{g} / \mathrm{g})$ or in weight percent. The sampling rate for the gas-liquid chromatograph is measured in hertz $(\mathrm{Hz})$ or one cycle per second.

Water temperature in degrees Celsius $\left({ }^{\circ} \mathrm{C}\right)$ can be converted to degrees Fahrenheit $\left({ }^{\circ} \mathrm{F}\right)$ by use of the following equation:

$$
{ }^{\circ} \mathrm{F}=1.8\left({ }^{\circ} \mathrm{C}\right)+32
$$




\title{
Geochemical Data on Concentrations of Inorganic Constituents and Polychlorinated Biphenyl Congeners in Streambed Sediments in Tributaries to Lake Champlain in New York, Vermont, and Quebec, 1992
}

\author{
By John A. Colman and Stewart F. Clark
}

\section{Abstract}

Geochemical data are presented from a survey of 42 inorganic constituents, polychlorinated biphenyl congeners, and organic carbon in streambed sediments of tributaries to Lake Champlain. The survey was completed during the summer of 1992 . The project was concerned with quantification of inputs of toxic constituents to Lake Champlain, such as polychlorinated biphenyl compounds (PCB's) and trace metals including mercury, from one potential source, the tributary streams. Sediment samples for both organic and inorganic analyses were taken from each of the 34 major tributary streams and from 39 minor tributary streams. The $\mathbf{3 4}$ major tributary streams drain 97 percent of the basin area. The additional 39 minor tributary streams were selected and sampled for inorganic (usually) or organic analysis to provide a more complete, areal coverage along the coastline of the lake. Tributary-stream-sampling sites were chosen close enough to the lake to capture as much upstream cultural influence as possible but with consideration of contamination by lake waters during periods of high lake level. The bed sediment of Lake Champlain was sampled for PCB analysis in 16 separate sites adjacent to the mouths of major tributaries but in water deep enough to be dominated by sediments in contact with lake water. The survey of streambed sediment was initiated to screen the many tributaries for potential input of low-solubility, toxic materials to the Lake. After screening, subsequent investigation could assess loads from a smaller number of tributaries that were found to contain significant concentrations of toxic constituents in the bed sediment. In the case of PCB's, the congener pattem could be compared among tributaries to distinguish types of sources.

\section{INTRODUCTION}

The geochemical investigation of streambed sediments of tributaries of Lake Champlain was initiated to support the agenda of the Lake Champlain Special Designation Act of 1991. The Act authorized a Management Conference Committee to convene to address lake management activities, among which were assessment of sources and cycling of toxic constituents in the lake. The investigation was concerned with quantification of inputs to Lake Champlain of toxic constituents, such as polychlorinated biphenyl compounds (PCB's) and trace metals, from one potential source, the tributary streams. PCB's and mercury are distinguished from other toxic materials measured in that some of their concentrations in fish flesh have exceeded the U.S. Food and Drug Administration advisory levels for safe consumption. An investigation of the sources of PCB's and mercury addresses the specific problem of toxic compounds in fish, as well as contributes to the general knowledge of the cycling of low solubility compounds of which these materials are examples.

External input rates and in-lake processing of toxic substances determine their ultimate impact in lakes. Measurement of toxic substance loads from potential sources is necessary for informed management of toxic substances. Depending on the relative magnitudes of the atmospheric, tributary, and directdischarge sources, it may be possible to reduce inputs by diversion, treatment, dredging, or other means.

The relative importance of the tributary load to Lake Champlain cannot be determined without comparing measurements from other sources. In other lakes, the ratio of atmospheric to non-atmospheric inputs depends on the presence of point sources, the 
ratio of drainage basin to lake area, and for trace metals, the ratio depends on the presence of natural sources (Swackhamer and Armstrong, 1987). For the Lake Champlain Basin there are various point sources from industry and publicly owned treatment plants. PCB's and trace metals persist in the environment; therefore, releases before the current (1994) accounting of point-source releases must also be considered. Natural sources of trace elements are in metal-sulfide deposits in the basin (Slack and Schruben, 1990). The atmosphere could be a significant source of toxic materials, particularly mercury, because atmospheric sources alone can cause mercury contamination of fish in oligotrophic lakes with low $\mathrm{pH}$ (Sorensen and others, 1990; Swain and Helwig, 1989). Although Lake Champlain is not oligotrophic and has bicarbonate buffering, atmospheric loading of mercury must be considered.

The narrow Champlain Valley has a large number of small tributaries that flow into the Lake from Vermont, New York, and Quebec (fig. 1, at back of report). The large number of tributaries make the measurement of their input load much more difficult than if one large river dominated. Because monitoring all of the tributaries is expensive, some screening is necessary to select which tributaries are most important in delivering substantial contaminant loads.

Streams may be efficiently surveyed for lowsolubility materials, such as trace elements and PCB's, by analysis of their bed material. Low-solubility substances partition to the solid phase so that concentrations of low-solubility materials in the bed material are many times larger than the concentrations of low-solubility materials in the water (Chao, 1984). The bed material also integrates the water-column concentrations, so that concentrations of toxic materials in sediment reflect concentrations in the water column that have occurred throughout the year. An efficient design for evaluating tributary loads of low-solubility substances would include an initial screening of tributaries through bed-sediment sampling. Subsequently, loads could be determined by water-column measurements made in tributaries chosen according to load potential as measured by their concentrations of sediment constituents.

For the PCB's, it may be possible to determine the number of sources or types of sources, as well as relative magnitude of the sources by surveying the streambed sediments. PCB's were pro- duced by chlorinating the biphenyl compound and collecting the various distillation fractions to obtain the final chlorinated biphenyl mixtures. The primary producer of PCB mixtures used in the United States marketed nine different PCB mixtures under the Aroclor ${ }^{1}$ trademark with levels of chlorination ranging from about 21 to 68 percent (Alford-Stevens, 1986). Each Aroclor mixture contained a characteristic mixture of PCB congeners. By comparing the congener pattern among samples, it may be possible to determine whether one or more sources of PCB are present in the tributaries. By comparing congener patterns in the lake sediments with those of tributaries, it may be possible to connect lake concentrations with tributary sources.

\section{Purpose and Scope}

The purpose of this report is to present geochemical data on inorganic constituents, organic carbon, and PCB congeners in the bed sediments of tributaries to Lake Champlain. The inorganic and carbon constituent data are listed as concentrations in the report tables. These data can be located in the tables by map site number, latitude and longitude, or by a remark code that indicates the purpose for collecting the sample. The PCB congeners are presented in histograms that show the congener concentration pattern of each sample. The scope of the report includes a presentation of the investigative design, methods, qualityassurance data, summary statistics, and results for the individual samples.

\section{Description of the Study Area}

\section{Hydrologic Setting}

The Lake Champlain drainage basin covers $8,277 \mathrm{mi}^{2}$ in northwestern Vermont, northeastern New York, and southern Quebec. Long, narrow, north-south trending Lake Champlain is $110 \mathrm{mi}$ long, up to $12 \mathrm{mi}$ wide, and locally as much as 400 $\mathrm{ft}$ deep (Meyer and Gruendling, 1979). Approximately two-thirds of its drainage basin is east of the Lake and one-third is west of the Lake.

\footnotetext{
${ }^{1}$ Use of trade names in this report is for identification purposes only and does not constitute endorsement by the U.S. Geological Survey.
} 
Henson and Gruendling (1977) tabulated 296 tributaries to Lake Champlain. Ten streams drain 80 percent of the Champlain basin, and the 24 largest streams drain 95 percent of the basin. Only 34 streams have a drainage area greater than $10 \mathrm{mi}^{2}$. Roughly 70 percent of the discharge to Lake Champlain comes from the eastern two-thirds of the basin. Thirty percent of the discharge comes from the western third of the basin (Hunt and others, 1972).

The water level of Lake Champlain is unregulated. Discharge from the Lake flows northward down the Richelieu River where a bedrock ridge controls the minimum lake-water level. Maximum water levels of Lake Champlain during the USGS record were approached in 1903 with a water level of $101.80 \mathrm{ft}$ and in 1993 with a water level of $101.88 \mathrm{ft}$. Minimum water level of $92.17 \mathrm{ft}$ was observed in 1941. The mean water level of Lake Champlain is $95 \mathrm{ft}$.

\section{Geologic Setting}

Five physiographic provinces are found in the basin. These provinces include the Adirondack Mountains to the west, the Green Mountains, and the Taconic Mountains to the east and south, the Vermont Valley, and the Champlain-St. Lawrence lowlands. The relief of these provincial regions ranges from low to moderate. Bedrock geology in each province is distinct.

The Adirondacks are underlain by coarse grained, metamorphic rocks that have been strongly altered from the form of their parent materials into their present condition. These rocks are now metagabbro, anorthosite, amphibolite, quartzite, marble, and other gneisses. In the Green Mountains, metamorphism of oceanic sediments produced phyllites, schists, and greenstones. The Taconic Mountains are composed of shales, slates, phyllites, and quartzites, which overlie limestones and sandstones of the Vermont Valley. Unmetamorphosed to weakly metamorphosed sedimentary limestones, dolomites, shales, and sandstones extend northward from the Vermont Valley through the Champlain Valley-St. Lawrence Lowland.

Bedrock variations define the physiographic provinces of the Lake Champlain Basin but unconsolidated stratified deposits cover much of the bedrock near Lake Champlain. These materials formed as shoreline, lake bottom, and ice-contact deposits during retreat of the continental ice sheet. Features associated with a freshwater glacial lake are overlain at lower altitudes by deposits from a saltwater sea connected to the Atlantic Ocean (Chapman, 1937, Wagner, 1972). These deposits locally influence the composition and texture of the sediments taken from the mouths of streams.

Four general geologic settings can be defined by conditions found at the mouths of streams entering Lake Champlain. These settings produce samples dominated by (1) Adirondack bedrock (mafic gneisses), (2) Champlain Valley bedrock (carbonates and sandstones), (3) unconsolidated silts and clays from the floor of glacial Lake Vermont or the floor of the Champlain Sea, and (4) samples dominated by fine to coarse grained sand and gravel deposited at the shoreline of glacial Lake Vermont.

\section{Acknowledgments}

The authors thank Captain Richard Furbush and his crew for their help aboard the University of Vermont's research vessel and Alan McIntosh for the arrangements he made for sampling the lake. Support for the project from a National Science Foundation scholarship to Shaili Pfeiffer was greatly appreciated. The assistance with data management from Jeremy Slaton was also appreciated.

\section{METHODS}

\section{Survey Design}

The survey of streambed sediment was initiated to screen the many tributaries for potential input of low-solubility toxic materials to Lake Champlain. After screening, subsequent investigation could assess loads from a smaller number of tributaries that were found to contain significant concentrations of toxic materials in the bed sediment. In the case of PCB's, the congener pattern could be compared among tributaries to distinguish the number and types of sources.

In order to intercept as many sources in a given tributary as possible, sampling sites were selected at the most downstream access point. However, contamination of stream sediments during times of high lake levels by materials of lake, rather than stream, origin was also considered in choosing sampling locations. Sampling sites were chosen at an altitude above $102 \mathrm{ft}$, except in lowgradient streams where the $102-\mathrm{ft}$ level was more 
than $1 \mathrm{mi}$ from the lake shore. The low-gradient reaches below the sampling sites often were associated with wetlands and were consequently in undeveloped areas. In the Saranac River, which flows through the city of Plattsburg just before the river enters the lake, an extra downstream sample was taken below the 102-ft level. Sites were selected upstream of highway, railroad, or power-line crossing of streams in order to avoid the local characteristics of these influences.

Locations of all sampling sites with associated site numbers are shown in figure 1 . Sites where metals were sampled are indicated with upward-pointing triangles, sites where PCB's were sampled with downward pointing triangles, and sites where both kinds of samples were taken are indicated with stars. Site number, name, latitude and longitude, and sample type of each site are given in table 1 (at back of report). Sediment samples were taken from each of the 34 major tributary streams, which drain 97 percent of the basin area and 39 minor tributary streams. Samples from the 34 major tributary streams were analyzed for organic and inorganic constituents. The additional 39 minor tributary stream sediments were selected and analyzed for inorganic (usually) or organic analysis to provide a more complete, areal coverage along the coastline of the lake. The bed sediment of Lake Champlain was sampled for PCB analysis in 16 separate sites adjacent to the mouths of major tributaries but in water deep enough to be dominated by sediments in contact with lake water.

At 10 inorganic and 17 organic sites, duplicate samples were collected by resampling the bed materials. One of each of the replicate inorganic pairs was split so that three samples would be submitted for analysis. For the inorganic analysis, the three samples were each split into between-site, sampling, and analytical components, after Ray (1982), to partition total variance associated with sample concentrations. Eight organic samples were split for analysis. For the organic analysis, congener patterns were compared visually to determine if the sample patterns were diagnostic.

The analysis of variance serves as a qualityassurance procedure. The presence of all or most of the variance between sites, rather than at a site, indicates the precision of the data. A second quality-assurance procedure used included standardreference materials in the samples submitted to the laboratory. The inorganic standards used were a reference shale sample (SDO-1) and a marine mud
(MAG-1) obtained from the USGS. The organic standard used was a marine sediment (HS-2) obtained from the Marine Analytical Chemistry Standards Program of the National Research Council, Canada.

\section{Field Methods}

Sites that could be reached by wading were approached from the downstream direction and sampled while wading upstream. Sandy, aerobic sediments, less than 2-mm in diameter that were not exposed by low water, were collected for the inorganic- and organic-constituent analyses. A stainless-steel scoop with a 3-ft-long stainless-steel handle was used to scrape the surface of sandy point-bar deposits. Care was taken to minimize loss of fine material to the stream water as the sampler was moved to the surface. Sites too deep to wade or that had difficult access were sampled from a canoe with a small stainless-steel Ekman dredge. A collected sample was deposited on a stainless-steel 2-mm mesh sieve. The sample was worked through the sieve with a stainless-steel mixing spoon into a shallow stainless-steel pan. Additional composite scoops were sieved as necessary until a 1-L microporous Tyvek bag could be filled with sediment. Surplus material was not gathered, so that the entire size fraction equal to or less than $2 \mathrm{~mm}$ was poured into the bag. The bag was then suspended from a rack and allowed to drip and air dry. After drying, samples for inorganic analysis were sent to the USGS laboratory in Denver, Colo. for further processing and chemical analysis.

A separate collection of sediment was made for analysis of organic constituents. Material collected for analysis of organics from tributary streams was processed in the same manner as material collected for trace-metal analysis but with a smaller volume $(300 \mathrm{~mL})$ of material. Sieved material for analysis of organics was transferred to a pre-baked, clean 500-mL glass jar with a Teflonlined lid and held on ice. Excess water was decanted when the sediment had settled. The sediment was then frozen in the jar.

Lake-bed sediments were sampled from the University of Vermont's research vessel, Melosira. A large steel Ekman dredge retrieved, landed, and released intact well layered slabs of lake-bed sediment into a stainless-steel pan. The upper layer was scooped with a stainless-steel spoon directly into pre-baked glass sample jars without sieving. Sam- 
ples for organic-constituent measurements were sent to the State University of New York at Syracuse for analysis.

Sample equipment was cleaned at each site with Liqinox detergent and rinsed thoroughly with river water. Equipment was cleaned after sampling and transported clean in plastic bags to the next site. Before sampling, equipment was rinsed again in river water.

At each stream site, $\mathrm{pH}$ and specific conductance were measured following sediment collection. The measurements were made according to standard USGS procedures (Fishman and Friedman, 1989).

\section{Chemical Analysis}

\section{Inorganlc Constlituents}

Upon receipt of the samples at the USGS laboratory in Denver, the dried stream samples were processed through a jaw crusher. The samples were then placed in a ceramic "juicer" (a commercial mechanical soil grinder) for further disaggregation with minimal particle disintegration. About 25 percent of the sample was separated out and archived. The remainder of the sample was dry sieved through a $63-\mu \mathrm{m}$ mesh stainless-steel sieve to separate the fine fraction for analysis.

Three techniques were used for the digestion of the resulting fine fraction-partial digestion for subsequent mercury analysis, partial digestion for subsequent 10-element analysis, and total digestion for subsequent analysis of 42 elements (Arbogast, 1990). The partial-digestion techniques were used because of the lower detection limits that are possible when the mineral-grain matrix is not dissolved. The partial digestion for mercury was by nitric acid and sodium dichromate in a closed Teflon vessel. The partial digestion for the 10-element analysis was with hydrochloric acid and hydrogen peroxide to solubilize metals that were not tightly bound in the silicate lattice of the sediments before analysis (Arbogast 1990). The total-digestion technique for the 42-element analysis used a mixture of hydrochloric, nitric, perchloric, and hydrofluoric acids.

All analyses were done by use of inductively coupled plasma-atomic emission spectrometry (ICP-AES) except for the mercury analysis, which was by cold vapor-atomic absorption spectrophotometry. Methods used are further described in
Arbogast (1990). Minimum reporting levels vary by method and element and are listed in table 2 (at back of report).

\section{Organic Constituents}

Analysis of PCB congeners was done at the State University of New York at Syracuse. Upon receipt at the laboratory, sample names and date of arrival were recorded and samples were stored in the dark at $4^{\circ} \mathrm{C}$. Each sample was prepared for analysis by drawing off supernatant water in the sample container, stirring the sediment to homogenize it, and placing the sediment on a $14.5-\mathrm{cm}$ diameter watch glass in a fume hood to air dry (4872 hours). The visual appearance of the sample (sandy, clayey, coarse, fine, organic matter) was recorded. After drying, the sample was divided into four quarters and each quarter was placed in a polyethylene Whirl-Pak bag. One quarter was sent to the USGS laboratory in Denver for organic carbon analysis and the others were stored at $4^{\circ} \mathrm{C}$.

The organic-carbon analysis was determined at the USGS Denver laboratory by difference between measurements of total carbon and inorganic carbon. The total carbon was measured by detection of carbon dioxide after combustion and inorganic carbon was measured by coulometric titration after acidification (Arbogast, 1990).

The PCB congeners were determined by gasliquid chromatography (GC). There is no standard USGS method for this analysis so the steps used for this report are described in the following paragraphs.

Approximately $10 \mathrm{~g}$ of sediment for extraction by solvent was weighed in a glass-soxhlet thimble. Distilled water was added until the sediment appeared damp (turned dark). A $100-\mu \mathrm{L}$ aliquot of toluene containing $52 \mathrm{ng} 2,2^{\prime}, 6,6^{\prime}-$ tetrachlorobiphenyl, $40 \mathrm{ng}$ 3,3',5,5'-tetrachlorobiphenyl, $54 \mathrm{ng}$ pentachlorobenzene, and $0.37 \mu \mathrm{g}$ of hexabromobiphenyl were added to the sediment, and the sample was extracted with 10 percent (volume basis) acetone in hexane in a soxhlet extractor for 24 hours. The refluxing solvent contained copper turnings to remove elemental sulfur during the extraction process. The copper was precleaned by sequential rinsing with 50 percent hydrochloric acid, distilled water, acetone, and hexane. A method blank (empty soxhlet thimble) was carried through with each batch of samples processed. After cooling, the extract was transferred to a Kud- 
erna-Danish concentrator and evaporated to approximately $2 \mathrm{~mL}$ on a steam bath.

The concentrated extract was placed on a column of $1 \mathrm{~g} \mathrm{60-100} \mathrm{mesh} \mathrm{florisil} \mathrm{with} \mathrm{approxi-}$ mately $15 \mathrm{~g}$ sodium sulfate on top in a Pasteur pipet and eluted with $15 \mathrm{~mL}$ hexane. Internal standard (67 ng octachlorostyrene and $315 \mathrm{ng}$ decachlorobiphenyl in $10 \mu \mathrm{L}$ toluene) and $2 \mathrm{~mL}$ isooctane were added to the sample and it was evaporated to $2 \mathrm{~mL}$ by bubbling a stream of charcoal-filtered air through it. Next, $5 \mathrm{~mL}$ concentrated sulfuric acid was added to the extract, and it was mixed with a vortex mixer for 1 minute. After standing for 1 minute, the solvent layer was transferred to an autoinjector vial with a Pasteur pipet. The acid layer was rinsed with $1 \mathrm{~mL}$ hexane and the hexane was transferred to the same vial.

Samples were analyzed with a GC equipped with an autoinjector, splitless capillary inlet, $30 \mathrm{~m}$ x $0.25 \mu \mathrm{m}$ SE-54 fused silica capillary column and ${ }^{63} \mathrm{Ni}$ electron capture detector (ECD). Digitized output (sampling rate of $2.5 \mathrm{~Hz}$ ) from the GC was sent through a RS-232 serial port to a microcomputer running a BASIC program to collect the data and store it in a disk file using the sample name as the file name.

At the beginning of each batch of samples analyzed on the $\mathrm{GC}$, five standards containing approximately $0.2,0.4,0.6,0.8$ and 1 parts per million (ppm) each of Aroclor 1242 and 1260 and the internal standards were analyzed. A standard containing approximately $1 \mathrm{ppm}$ of each Aroclor (1242 and 1260) was run after every five samples. Identities and quantities of congeners in the standards were established on the basis of the work of Schulz and others (1989). Response factors relative to the internal standards were determined from the first five standards and averaged. The average and standard deviation of the retention time of each congener was determined from the first five standards. Congeners were identified by use of retention-time windows bounded by plus and minus three standard deviations of the mean retention time. Congeners were quantified by use of peak heights relative to the internal standard-peak height and the relative-response factors. Peak height, retention time, identification, and quantification were all determined by use of Labtech Chrom version 2 to process the raw GC data.

In the United States, PCB's were used primarily as Aroclors each of which contained a characteristic mixture of congeners. PCB patterns found in the environment generally reflect a mixture of different Aroclors with some modification as a result of weathering. Therefore, the presence of a pattern of peaks similar to an Aroclor or mixture of Aroclors indicates the presence of PCB's. Analytical results were screened to determine if the major congeners in each Aroclor (congeners 31 and 28 for $1242 ; 66$ and 95 for $1248 ; 77$ and 110 for 1254 and 180 for 1260 ) were present in the samples. Samples with no peaks corresponding to any of these congeners present at levels that would correspond to $0.01 \mathrm{ppm}$ of the Aroclor in the sample were recorded as samples with no detected PCB's.

\section{SUMMARY STATISTICS AND GEOCHEMICAL DATA}

\section{Inorganic Constituents}

Results of the standard reference-material analyses and corresponding standard concentrations are presented in table 3 (in back of report). Most standard concentrations given represent results from five or more laboratories and three or more independent methods in statistical agreement. Element standard concentrations not represented by well established independent methods are indicated with footnotes. Of the 30 elements quantified in the standards, only two comparisons between reference material and analysis were outside the 2 standard-deviation-unit range. These were the comparison of barium in the SDO-1 sample and of copper in the MAG-1 sample.

Percent variance of ranked data at each level of the analysis of variance design is presented in table 4 (in back of report). Variation introduced by the analytical method and sample splitting as a percentage of total variance is shown in the column under "Sample split." Variation introduced by the stream-sampling procedure is shown in the column under "Within site," and variation found among sites is shown in the column under "Between site." Elements for which virtually all of the results were below detection are not included in the table.

Summary statistics of the inorganic-analysis results were computed on the environmental samples, one sample from each site. Percentile data and maximum and minimum results are presented in table 5 (in back of report). A percentile value has at most the given percent of observations less than the 
value and 100 minus the given percent of observations greater than the value.

Concentrations for all elements by sample site number are shown in table 6 (in back of report). The site numbers refer to figure 1 , where sampling sites are shown. Map numbers are consecutive, sorted in order of decreasing latitude. Each sample in table 6 is listed with a "Design remark" that refers to the intended use of the sample in the survey design.

\section{Organic Constituents}

Not all of the PCB congeners can be separated with the chromatographic conditions used in this study. Although most of the congeners are uniquely determined by retention time, it is possible for as many as three congeners to elute from the column at the same time. Information on the degree to which congeners are resolved is given in table 7 (in back of report) where the congeners and their International Union of Practical and Applied Chemistry (IUPAC) congener numbers are listed in order of their retention time in the GC. Each PCB peak that can be resolved is assigned a sequential domain number so that congeners in table 7 that coelute have the same domain number.

Results of the standard reference-material analyses and corresponding certified concentrations are presented in table 8 (in back of report). Only 2 of 10 congeners that were certified in the standard were uniquely defined by a domain. Results are from two samples of the reference material analyzed as blind samples by the laboratory. Although the sample size is too small to be conclusive regarding the accuracy of the analysis, results in general agree with the standard.

Data on sampling design for organic sample sites is given in table 9 (in back of report). The site numbers refer to figure 1 . Results of the analyses of carbonate, organic, and total carbon are also included in table 9. These samples were splits of those analyzed for PCB's.

Results of the PCB congener-sample analyses are given in table 9 and figure 2 (in back of report). Not all of the samples had measurable concentrations of PCB's in them. Those samples that did not meet the concentration criterion described in the methods section have "no" in the last column of table 9 indicating that PCB's were not detected. Those samples that did meet the concentration cri- terion for detection are indicated by "yes" in the last column.

In some cases, one replicate from a site was classified as showing a PCB congener pattern, whereas another replicate from the same site was classified as not showing a PCB congener pattern. Variation in samples at a site and analytical variation can cause this difference in classification when the amount of PCB's in the sample is small. The pattern of congeners for samples with detections is given in figure 2 . The results are in histogram, rather than table form, to emphasize the pattern of PCB congener occurrence among samples. The response axes of the histograms are given in terms of concentration. The nominal concentration units of the figure would correspond to actual concentration in micrograms per gram dry-weight basis if the height of the peak were caused only by PCB congeners. However, this is not necessarily the case since non-PCB compounds can be registered at the ECD. The sample treatment and clean up, and the ECD that was used, are selective for PCB compounds. However, other non-PCB compounds, not removed from the sample extract by the cleanup steps, may respond in the ECD and interfere with the PCB analysis. The interference produces either false identifications or concentrations that are biased high because of the interfering compound's response contribution. As discussed in the section "Methods," a pattern of peaks indicate the presence of PCB's, but for any one peak, only tentative identification is possible. The histograms in figure 2 are arranged in order of sample-site number. With the site number on each histogram is the design remark code from table 9 indicating duplicate and split samples.

Congener patterns of the principal PCB Aroclor mixtures that were used in the United States are given in figure 3. A comparison between patterns of the Aroclor and the environmental samples can be done to determine how much the environmental samples differ from the original patterns.

\section{SUMMARY}

This report does not include an interpretation of the large data set presented in the report tables. However, the tables contain numerous data on the distribution and presence of organic and inorganic constituents in the Lake Champlain tributaries. 


\section{REFERENCES}

Alford-Stevens, A.L., 1986, Analyzing PCB's: Environmental Science and Technology, v. 20, p. 11941199.

Arbogast, B.F., 1990, Quality assurance manual for the Branch of Geochemistry, U.S. Geological Survey: U. S. Geological Survey Open-File Report 90-688, $184 \mathrm{p}$.

Chapman, D.H., 1937, Late-glacial and post-glacial history of the Champlain Valley: American Journal of Science, v. 34, p. 89-124.

Chao, T.T., 1984, Use of partial dissolution techniques in geochemical exploration: Journal of Geochemical Exploration, v. 20, p. 101-135.

Fishman, M.J. and Friedman, L.C., 1989, Methods for determination of inorganic substances in water and fluvial sediments: U. S. Geological Survey, Techniques of Water-Resources Investigations, chap. A1, book 5, $545 \mathrm{p}$.

Henson, E.B. and Gruendling, G.K., 1977, The Trophic status and phosphorus loadings of Lake Champlain: Boston, Mass. and Corvallis, Oreg., EPA-600/3-77$106,115 \mathrm{p}$.

Hunt, A.S., Henson, E.B., and Bucke, D.P., 1972, Sedimentological and limnological studies of Lake Champlain, in Doolan, B.L. and Stanley, R.S., ed., New England Intercollegiate Conference, 64th Annual meeting, Burlington, Vt., October 12, 1972, Guidebook: University of Vermont, p. 407-426.

Myer, G.E. and Gruendling, G.K., 1979, Limnology of Lake Champlain: New England River Basins Commission, $407 \mathrm{p}$.

Ray, A., ed., 1982, SAS users guide--Statistics: Cary, N.C., SAS Institute Inc., 584 p.

Schulz, D.E., Petrick, G., and Duinker, J.C., 1989, Complete characterization of polychlorinated biphenyl congeners in commercial aroclor and clophen mixtures by multidimensional gas chromatographyelectron capture detection: Environmental Science and Technology, v. 23, p. 852-859.

Slack, J.F., and Schruben, 1990, Metallic mineral deposits in the Glens Falls 1 degree $\mathrm{x} 2$ degree quadrangle, New York, Vermont, and New Hampshire in Slack, J.F., ed., Summary results of the Glens Falls CUSMAP project, New York, Vermont, and New Hampshire-Geologic, geophysical, and geochemical studies related to metallic mineral resources in westcentral New England: U.S. Geological Survey Bulletin 1887, chap. H, H1-H6 p.

Sorensen, J.A., Glass, G.E., Schmidt, K.W., Huber, J.K., and Rapp, G.R., 1990, Airborne mercury deposition and watershed characteristics in relation to mercury concentrations in water, sediments, plankton, and fish of eighty northern Minnesota lakes: Environmental Science and Technology, v. 24, p. 17161727.

Swackhamer, D.L. and Armstrong, D.E., 1987, Distribution and characterization of PCB's in Lake Michigan water: Journal of Great Lakes Research, v. 13, p. 24-36.

Swain, E.B. and Helwig, D.D., 1989, Mercury in fish from northeastern Minnesota lakes-Historical trends, environmental correlates, and potential sources: Journal of the Minnesota Academy of Science, v. 55, p. 103-109.

Wagner, W.P., 1972, Ice margins and water levels in northwestern Vermont, in Doolan, B.L. and Stanley, R.S., eds., New England Intercollegiate Conference, 64th Annual meeting, Burlington, Vt., October 12, 1972, Guidebook: University of Vermont, p. 319342. 
Table 1. Location and sample-type data for sites sampled in the Lake Champlain Basin

\begin{tabular}{|c|c|c|c|c|}
\hline $\begin{array}{c}\text { Site } \\
\text { numbe }\end{array}$ & Stream name & Latitude & Longitude & Sample type \\
\hline 1 & Pike River & $45^{\circ} 04^{\prime} 40.3 "$ & $73^{\circ} 05^{\prime} 19.0 "$ & inorganic, organic \\
\hline 2 & Lake Bed near Pike River & $45^{\circ} 03 \cdot 32.8^{\prime \prime}$ & $73^{\circ} 06^{\circ} 00.0^{\prime \prime}$ & organic \\
\hline 3 & Lake Bed near Missisquoi River & $45^{\circ} 01 \cdot 18.7 "$ & $73^{\circ} 09^{\prime} 11.9 "$ & organic \\
\hline 4 & Rock River & $44^{\circ} 59^{\prime} 46.9^{\prime \prime}$ & $73^{\circ} 04^{\prime} 23.4 "$ & inorganic, organic \\
\hline 5 & Carman Brook & $44^{\circ} 58 \cdot 38.1 "$ & $73^{\circ} 05^{\prime} 01.8 "$ & inorganic \\
\hline 6 & Mud Creek & $44^{\circ} 58^{\prime} 05.5^{\prime \prime}$ & $73^{\circ} 16^{\prime} 10.5^{\prime \prime}$ & inorganic \\
\hline 7 & Mud Creek & $44^{\circ} 57^{\prime} 51.0 "$ & $73^{\circ} 16^{\prime} 12.8 "$ & organic \\
\hline 8 & Youngman Brook & $44^{\circ} 57^{\prime} 22.7 "$ & $73^{\circ} 06^{\prime} 23.6^{\prime \prime}$ & inorganic \\
\hline 9 & Great Chazy River & $44^{\circ} 57^{\prime} 13.1 "$ & $73^{\circ} 25^{\prime} 03.8 "$ & inorganic, organic \\
\hline 10 & Corbeau Creek & $44^{\circ} 56^{\prime} 27.2^{\prime \prime}$ & $73^{\circ} 24 \cdot 36.0 "$ & inorganic \\
\hline 11 & Missisquoi River & $44^{\circ} 55^{\prime} 53.8^{\prime \prime}$ & $73^{\circ} 07^{\prime} 37.7^{\prime \prime}$ & inorganic, organic \\
\hline 12 & Lake Bed near Great Chazy River & $44^{\circ} 55^{\prime} 30.0 "$ & $.00 .0 "$ & organic \\
\hline 13 & Little Chazy River & $44^{\circ} 54^{\prime} 10.0^{\prime \prime}$ & $73^{\circ} 24^{\prime} 52.9 "$ & inorganic, organic \\
\hline 14 & Stephens Brook & $44^{\circ} 50^{\prime} 56.6^{\prime \prime}$ & $73^{\circ} 07^{\prime} 08.0^{\prime \prime}$ & inorganic, organic \\
\hline 15 & Stephens Brook & $44^{\circ} 50^{\prime} 50.0^{\prime \prime}$ & $73^{\circ} 05^{\prime} 52.0^{\prime \prime}$ & inorganic \\
\hline 16 & Riley Brook & $44^{\circ} 48^{\prime} 28.0^{\prime \prime}$ & $73^{\circ} 24^{\prime} 03.0^{\prime \prime}$ & inorganic, organic \\
\hline 17 & Lake Bed in st. Albans Bay & $44^{\circ} 47^{\prime} 17.2^{\prime \prime}$ & $73^{\circ} 09^{\prime} 24.1 "$ & organic \\
\hline 18 & Mill River & $44^{\circ} 46^{\prime} 47.0^{\prime \prime}$ & $73^{\circ} 08^{\prime} 39.2^{\prime \prime}$ & inorganic, organic \\
\hline 19 & Dead Creek & $44^{\circ} 45^{\prime} 31.4^{\prime \prime}$ & $73^{\circ} 26^{\prime} 51.5^{\prime \prime}$ & inorganic, organic \\
\hline 20 & Ray Brook & $44^{\circ} 45^{\prime} 09.7 "$ & $73^{\circ} 27^{\prime} 48.6^{\prime \prime}$ & inorganic \\
\hline 21 & Kennon Brook & $44^{\circ} 44^{\prime} 12.5^{\prime \prime}$ & $73^{\circ} 28^{\prime} 18.1 "$ & inorganic \\
\hline 22 & Dead Creek Marsh & $44^{\circ} 43^{\prime} 21.5^{\prime \prime}$ & $73^{\circ} 27^{\prime} 15.5^{\prime \prime}$ & organic \\
\hline 23 & unnamed tributary & $44^{\circ} 42^{\prime} 34.3^{\prime \prime}$ & $73^{\circ} 26^{\prime} 58.2^{\prime \prime}$ & organic \\
\hline 24 & Lake Bed in Cumberland Bay & $44^{\circ} 42^{\prime} 31.7 "$ & $73^{\circ} 26^{\prime} 45.0^{\prime \prime}$ & organic \\
\hline 25 & Lake Bed in Cumberland Bay & $44^{\circ} 42^{\prime} 03.4^{\prime \prime}$ & $73^{\circ} 25^{\prime} 19.0 "$ & organic \\
\hline 26 & Saranac River & $44^{\circ} 41^{\prime} 57.6 \prime$ & $73^{\circ} 26^{\prime} 58.5^{\prime \prime}$ & inorganic, organic \\
\hline 27 & Saranac River & $44^{\circ} 41^{\prime} 27.8^{\prime \prime}$ & $73^{\circ} 27^{\prime} 48.5^{\prime \prime}$ & organic \\
\hline 28 & Saranac River & $44^{\circ} 41^{\prime} 27.1^{\prime \prime}$ & $73^{\circ} 27^{\prime} 45.7 "$ & organic \\
\hline 29 & Stone Bridge Brook & $44^{\circ} 41^{\prime} 14.4 \prime$ & $73^{\circ} 11^{\prime} 22.6 "$ & inorganic, organic \\
\hline 30 & unnamed tributary & $44^{\circ} 39^{\prime} 12.1 "$ & $73^{\circ} 26^{\prime} 41.0^{\prime \prime}$ & inorganic, organic \\
\hline 31 & Trout Brook & $44^{\circ} 39^{\prime} 11.3 \prime$ & $73^{\circ} 12^{\prime} 58.9^{\prime \prime}$ & inorganic \\
\hline 32 & Salmon River & $44^{\circ} 37^{\prime} 41.2^{\prime \prime}$ & $73^{\circ} 26^{\prime} 55.0^{\prime \prime}$ & inorganic, organic \\
\hline 33 & Silver Stream & $44^{\circ} 37^{\prime} 14.0^{\prime \prime}$ & $73^{\circ} 26^{\prime} 52.8^{\prime \prime}$ & inorganic \\
\hline 34 & Lamoille River & $44^{\circ} 36^{\prime} 52.9^{\prime \prime}$ & $73^{\circ} 10^{\prime} 50.6^{\prime \prime}$ & inorganic, organic \\
\hline 35 & Lake Bed in Malletts Bay & $44^{\circ} 36^{\prime} 38.2 \prime$ & $73^{\circ} 15^{\prime} 02.9 "$ & organic \\
\hline 36 & Little Ausable River & $44^{\circ} 34^{\prime} 58.2 \prime$ & $73^{\circ} 27^{\prime} 29.6^{\prime \prime}$ & inorganic, organic \\
\hline 37 & Allen Brook & $44^{\circ} 34^{\prime} 43.3^{\prime \prime}$ & $73^{\circ} 09^{\prime} 28.6^{\prime \prime}$ & inorganic \\
\hline 38 & Lake Bed in Malletts Bay & $44^{\circ} 34^{\prime} 09.0^{\prime \prime}$ & $73^{\circ} 12^{\prime} 35.9 "$ & organic \\
\hline 39 & Malletts Creek & $44^{\circ} 34^{\prime} 00.9^{\prime \prime}$ & $73^{\circ} 09^{\prime} 16.2^{\prime \prime}$ & inorganic, organic \\
\hline 40 & Lake Bed near Ausable River & $44^{\circ} 33^{\prime} 57.4 "$ & $73^{\circ} 24^{\prime} 45.7^{\prime \prime}$ & organic \\
\hline
\end{tabular}


Table 1. Location and sample-type data for sites sampled in the Lake Champlain Basin-Continued

\begin{tabular}{|c|c|c|c|c|}
\hline $\begin{array}{c}\text { Site } \\
\text { number }\end{array}$ & Stream name & Latitude & Longitude & Sample type \\
\hline 41 & Indian Brook & $44^{\circ} 33^{\prime} 33.8 "$ & $73^{\circ} 10^{\prime} 50.8^{\prime \prime}$ & inorganic, organic \\
\hline 42 & Pond Brook & $44^{\circ} 33^{\prime} 30.5^{\prime \prime}$ & $73^{\circ} 09^{\prime} 52.9^{\prime \prime}$ & inorganic \\
\hline 43 & Ausable River & $44^{\circ} 33^{\prime} 19.5 "$ & $73^{\circ} 27^{\prime} 26.4^{\prime \prime}$ & inorganic, organic \\
\hline 44 & Winooski River & $44^{\circ} 32 \cdot 20.7 "$ & $73^{\circ} 15^{\prime} 56.0^{\prime \prime}$ & inorganic, organic \\
\hline 45 & Lake Bed near Winooski River & $44^{\circ} 31^{\prime} 36.2 "$ & $73^{\circ} 18^{\prime} 22.5^{\prime \prime}$ & organic \\
\hline 46 & Winooski River & $44^{\circ} 29^{\prime} 20.2 "$ & $73^{\circ} 11^{\prime} 03.1 "$ & inorganic, organic \\
\hline 47 & Little Trout Brook & $44^{\circ} 28^{\prime} 49.0^{\prime \prime}$ & $73^{\circ} 25^{\prime} 06.7^{\prime \prime}$ & inorganic \\
\hline 48 & Potash Brook & $44^{\circ} 26^{\prime} 22.6^{\prime \prime}$ & $73^{\circ} 13^{\prime} 10.7 "$ & inorganic \\
\hline 49 & unnamed tributary & $44^{\circ} 25^{\prime} 37.2 "$ & $73^{\circ} 24^{\prime} 51.0^{\prime \prime}$ & inorganic, organic \\
\hline 50 & Lake Bed in Shelburne Bay & $44^{\circ} 24 \cdot 31.6 "$ & $73^{\circ} 14^{\prime} 09.0 \prime$ & organic \\
\hline 51 & Munroe Brook & $44^{\circ} 24 \cdot 16.1 "$ & $73^{\circ} 13^{\prime} 03.0^{\prime \prime}$ & inorganic \\
\hline 52 & Laplatte River & $44^{\circ} 23^{\prime} 05.0^{\prime \prime}$ & $73^{\circ} 13^{\prime} 26.8^{\prime \prime}$ & inorganic, organic \\
\hline 53 & Bouquet River & $44^{\circ} 22 \cdot 10.8 "$ & $73^{\circ} 23^{\prime} 28.0^{\prime \prime}$ & inorganic, organic \\
\hline 54 & Lake Bed near Bouquet River & $44^{\circ} 21^{\prime} 27.5^{\prime \prime}$ & $73^{\circ} 21^{\prime} 00.3^{\prime \prime}$ & organic \\
\hline 55 & Holmes Creek & $44^{\circ} 19^{\prime} 50.6 "$ & $73^{\circ} 16^{\prime} 34.5^{\prime \prime}$ & inorganic \\
\hline 56 & Thorp Brook & $44^{\circ} 16^{\prime} 27.2^{\prime \prime}$ & $73^{\circ} 15^{\prime} 20.5^{\prime \prime}$ & inorganic \\
\hline 57 & Lewis Creek & $44^{\circ} 14^{\prime} 45.9 "$ & $73^{\circ} 14^{\prime} 44.5^{\prime \prime}$ & inorganic, organic \\
\hline 58 & Lake Bed near Otter Creek & $44^{\circ} 13^{\prime} 42.0^{\prime \prime}$ & $73^{\circ} 19^{\prime} 54.5^{\prime \prime}$ & organic \\
\hline 59 & Little Otter Creek & $44^{\circ} 11^{\prime} 53.8^{\prime \prime}$ & $73^{\circ} 14^{\prime} 46.2^{\prime \prime}$ & inorganic, organic \\
\hline 60 & Lake Bed near Hoisington Brook & $44^{\circ} 11^{\prime} 10.6 "$ & $73^{\circ} 24^{\prime} 46.2^{\prime \prime}$ & organic \\
\hline 61 & Hoisington Brook & $44^{\circ} 11^{\prime} 00.2^{\prime \prime}$ & $73^{\circ} 26^{\prime} 05.2^{\prime \prime}$ & ganic, organic \\
\hline 62 & Otter Creek & $44^{\circ} 09^{\prime} 27.0 "$ & $73^{\circ} 17^{\prime} 01.0 \prime$ & inorganic, organic \\
\hline 63 & Stacy Brook & $44^{\circ} 08^{\prime} 33.6^{\prime \prime}$ & $73^{\circ} 25^{\prime} 51.8^{\prime \prime}$ & inorganic \\
\hline 64 & Mullen Brook & $44^{\circ} 06^{\prime} 38.0 \prime$ & $73^{\circ} 27^{\prime} 04.0^{\prime \prime}$ & inorganic \\
\hline 65 & unnamed tributary & $44^{\circ} 06^{\prime} 06.0 "$ & $73^{\circ} 27^{\prime} 03.0^{\prime \prime}$ & inorganic \\
\hline 66 & Mullen Brook & $44^{\circ} 06^{\prime} 04.7 "$ & $73^{\circ} 27^{\prime} 00.0^{\prime \prime}$ & ino \\
\hline 67 & Mill Brook & $44^{\circ} 03^{\prime} 10.0 "$ & $73^{\circ} 27^{\prime} 22.6^{\prime \prime}$ & inorganic, organic \\
\hline 68 & Mill Brook & $44^{\circ} 02^{\prime} 58.0 "$ & $73^{\circ} 28^{\prime} 08.0^{\prime \prime}$ & inorganic \\
\hline 69 & McKenzie Brook & $44^{\circ} 02 \cdot 08.3 "$ & $73^{\circ} 27^{\prime} 49.2^{\prime \prime}$ & inorganic \\
\hline 70 & E. Branch Dead Creek & $44^{\circ} 011^{\prime} 41.8 "$ & $73^{\circ} 19^{\prime} 15.6^{\prime \prime}$ & inorganic \\
\hline 71 & West Branch Dead Creek & $44^{\circ} 01^{\prime} 32.5^{\prime \prime}$ & $73^{\circ} 20^{\prime} 53.6^{\prime \prime}$ & ins \\
\hline 72 & Grove Brook & $44^{\circ} 00 \prime 39.0^{\prime \prime}$ & $73^{\circ} 27^{\prime} 24.9^{\prime \prime}$ & inorganic \\
\hline 73 & Putnam Creek & $43^{\circ} 57^{\prime} 21.2^{\prime \prime}$ & $73^{\circ} 25^{\prime} 59.4^{\prime \prime}$ & inorganic, organic \\
\hline 74 & Lake Bed near Putnam Creek & $43^{\circ} 57^{\prime} 09.5^{\prime \prime}$ & $73^{\circ} 24^{\prime} 27.5^{\prime \prime}$ & organic \\
\hline 75 & Grant Brook & $43^{\circ} 54^{\prime} 24.2^{\prime \prime}$ & $73^{\circ} 25^{\prime} 25.7^{\prime \prime}$ & inorganic \\
\hline 76 & Five Mile Creek & $43^{\circ} 53^{\prime} 43.1^{\prime \prime}$ & $73^{\circ} 24^{\prime} 17.1^{\prime \prime}$ & nic \\
\hline 77 & unnamed tributary & $43^{\circ} 51^{\prime} 00.0^{\prime \prime}$ & $73^{\circ} 21^{\prime} 50.8^{\prime \prime}$ & inorganic \\
\hline 78 & Ticonderoga Creek & $43^{\circ} 50^{\prime} 49.3^{\prime \prime}$ & $73^{\circ} 24 \cdot 37.9 "$ & inorganic, organic \\
\hline 79 & Lake Bed near Lachutte & $43^{\circ} 50^{\prime} 05.3^{\prime \prime}$ & $73^{\circ} 23^{\prime} 19.6^{\prime \prime}$ & organic \\
\hline 80 & East Creek & $43^{\circ} 48^{\prime} 13.5^{\prime \prime}$ & $73^{\circ} 20^{\prime} 16.2^{\prime \prime}$ & inorganic, organic \\
\hline
\end{tabular}


Table 1. Location and sample-type data for sites sampled in the Lake Champlain Basin-Continued

\begin{tabular}{|c|c|c|c|c|}
\hline $\begin{array}{l}\text { Site } \\
\text { number }\end{array}$ & Stream name & Latitude & Longitude & Sample type \\
\hline 81 & Charter Brook & $43^{\circ} 48^{\prime} 04.6^{\prime \prime}$ & $73^{\circ} 23^{\prime} 42.8^{\prime \prime}$ & inorganic \\
\hline 82 & South Brook & $43^{\circ} 46^{\prime} 19.2^{\prime \prime}$ & $73^{\circ} 23^{\prime} 21.0^{\prime \prime}$ & inorganic \\
\hline 83 & Mill Brook & $43^{\circ} 43^{\prime} 59.2^{\prime \prime}$ & $73^{\circ} 23 \cdot 35.7 "$ & inorganic, organic \\
\hline 84 & unnamed tributary & $43^{\circ} 43^{\prime} 28.3^{\prime \prime}$ & $73^{\circ} 21^{\prime} 52.5^{\prime \prime}$ & inorganic \\
\hline 85 & unnamed tributary & $43^{\circ} 40^{\prime} 04.6^{\prime \prime}$ & $73^{\circ} 25^{\prime} 04.5^{\prime \prime}$ & inorganic \\
\hline 86 & Horton Brook & $43^{\circ} 38^{\prime} 55.9^{\prime \prime}$ & $73^{\circ} 23^{\prime} 35.9^{\prime \prime}$ & inorganic \\
\hline 87 & unnamed tributary & $43^{\circ} 38^{\prime} 15.6^{\prime \prime}$ & $73^{\circ} 26^{\prime} 23.1^{\prime \prime}$ & inorganic \\
\hline 88 & Hubbardton River & $43^{\circ} 37^{\prime} 37.5^{\prime \prime}$ & $73^{\circ} 20^{\prime} 33.2^{\prime \prime}$ & inorganic \\
\hline 89 & Poultney River & $43^{\circ} 37^{\prime} 33.9^{\prime \prime}$ & $73^{\circ} 20^{\prime} 35.5^{\prime \prime}$ & inorganic, organic \\
\hline 90 & Coggman Creek & $43^{\circ} 37^{\prime} 32.9^{\prime \prime}$ & $73^{\circ} 22^{\prime} 16.8^{\prime \prime}$ & inorganic \\
\hline 91 & Pine Lake Brook & $43^{\circ} 37^{\prime} 07.1^{\prime \prime}$ & $73^{\circ} 26^{\prime} 23.9^{\prime \prime}$ & inorganic \\
\hline 92 & Pike Brook & $43^{\circ} 33^{\prime} 02.4^{\prime \prime}$ & $73^{\circ} 27 \cdot 55.1^{\prime \prime}$ & inorganic \\
\hline 931 & Mud Brook & $43^{\circ} 32^{\prime} 42.5^{\prime \prime}$ & $73^{\circ} 23^{\prime} 41.7^{\prime \prime}$ & inorganic \\
\hline 94 & Greenland Brook & $43^{\circ} 32^{\prime} 06.4^{\prime \prime}$ & $73^{\circ} 30^{\prime} 22.5^{\prime \prime}$ & inorganic \\
\hline 95 & Metawee River & $43^{\circ} 31^{\prime} 46.6^{\prime \prime}$ & $73^{\circ} 23^{\prime} 25.8^{\prime \prime}$ & inorganic, organic \\
\hline 96 & Spectacle Brook & $43^{\circ} 31^{\prime} 23.3^{\prime \prime}$ & $73^{\circ} 30^{\prime} 35.6^{\prime \prime}$ & inorganic \\
\hline 97 & Mount Hope Brook & $43^{\circ} 31 ' 19.8 "$ & $73^{\circ} 30^{\prime} 29.5^{\prime \prime}$ & inorganic, organic \\
\hline
\end{tabular}


Table 2. Minimum reporting levels for the streambed sediments survey of the Lake Champlain Basin $[\mu \mathrm{g} / \mathrm{g}$, micrograms per gram]

\begin{tabular}{|c|c|c|c|c|c|}
\hline Element & Units & $\begin{array}{c}\text { Minimum } \\
\text { reporting } \\
\text { level }\end{array}$ & Element & Units & $\begin{array}{c}\text { Minimum } \\
\text { reporting } \\
\text { level }\end{array}$ \\
\hline Aluminum & percent & 0.1 & Tantalum & $\mu \mathrm{g} / \mathrm{g}$ & 40 \\
\hline Antimony - partial & $\mu \mathrm{g} / \mathrm{g}$ & .6 & Thorium & $\mu \mathrm{g} / \mathrm{g}$ & 4 \\
\hline Arsenic & $\mu \mathrm{g} / \mathrm{g}$ & 10 & Tin & $\mu \mathrm{g} / \mathrm{g}$ & 5 \\
\hline Arsenic - partial & $\mu \mathrm{g} / \mathrm{g}$ & .6 & Titanium & percent & .005 \\
\hline \multirow[t]{2}{*}{ Barium } & $\mu \mathrm{g} / \mathrm{g}$ & 1 & & & \\
\hline & & & Uranium & $\mu \mathrm{g} / \mathrm{g}$ & 100 \\
\hline Beryllium & $\mu \mathrm{g} / \mathrm{g}$ & 1 & Vanadium & $\mu \mathrm{g} / \mathrm{g}$ & 2 \\
\hline Bismuth & $\mu \mathrm{g} / \mathrm{g}$ & 10 & Ytterbium & $\mu \mathrm{g} / \mathrm{g}$ & 1 \\
\hline Bismuth - partial & $\mu \mathrm{g} / \mathrm{g}$ & .6 & Yttrium & $\mu \mathrm{g} / \mathrm{g}$ & 2 \\
\hline Cadmium & $\mu \mathrm{g} / \mathrm{g}$ & 2 & Zinc & $\mu \mathrm{g} / \mathrm{g}$ & 4 \\
\hline \multirow[t]{2}{*}{ Cadmium - partial } & $\mu \mathrm{g} / \mathrm{g}$ & .05 & & & \\
\hline & & & Zinc - partial & $\mu \mathrm{g} / \mathrm{g}$ & .5 \\
\hline Calcium & percent & .05 & & & \\
\hline Cerium & $\mu \mathrm{g} / \mathrm{g}$ & 4 & & & \\
\hline Chromium & $\mu \mathrm{g} / \mathrm{g}$ & 1 & & & \\
\hline Cobalt & $\mu \mathrm{g} / \mathrm{g}$ & 1 & & & \\
\hline Copper & $\mu \mathrm{g} / \mathrm{g}$ & 1 & & & \\
\hline Copper - partial & $\mu \mathrm{g} / \mathrm{g}$ & .05 & & & \\
\hline Europium & $\mu \mathrm{g} / \mathrm{g}$ & 2 & & & \\
\hline Gallium & $\mu \mathrm{g} / \mathrm{g}$ & 4 & & & \\
\hline Gold & $\mu \mathrm{g} / \mathrm{g}$ & 8 & & & \\
\hline Gold - partial & $\mu \mathrm{g} / \mathrm{g}$ & .1 & & & \\
\hline Holmium & $\mu \mathrm{g} / \mathrm{g}$ & 4 & & & \\
\hline Iron & percent & .05 & & & \\
\hline Lanthanum & $\mu \mathrm{g} / \mathrm{g}$ & 2 & & & \\
\hline Lead & $\mu \mathrm{g} / \mathrm{g}$ & 4 & & & \\
\hline Lead - partial & $\mu \mathrm{g} / \mathrm{g}$ & .6 & & & \\
\hline Lithium & $\mu \mathrm{g} / \mathrm{g}$ & 2 & & & \\
\hline Magnesium & percent & .005 & & & \\
\hline Manganese & $\mu \mathrm{g} / \mathrm{g}$ & 4 & & & \\
\hline Mercury - partial & $\mu \mathrm{g} / \mathrm{g}$ & .02 & & & \\
\hline Molybdenum & $\mu \mathrm{g} / \mathrm{g}$ & 2 & & & \\
\hline Molybdenum - partial & $\mu \mathrm{g} / \mathrm{g}$ & .09 & & & \\
\hline Neodymium & $\mu \mathrm{g} / \mathrm{g}$ & 4 & & & \\
\hline Nickel & $\mu \mathrm{g} / \mathrm{g}$ & 2 & & & \\
\hline Niobium & $\mu \mathrm{g} / \mathrm{g}$ & 4 & & & \\
\hline Phosphorus & percent & .005 & & & \\
\hline Potassium & percent & .05 & & & \\
\hline Scandium & $\mu \mathrm{g} / \mathrm{g}$ & 2 & & & \\
\hline Silver & $\mu \mathrm{g} / \mathrm{g}$ & 2 & & & \\
\hline Silver - partial & $\mu \mathrm{g} / \mathrm{g}$ & .06 & & & \\
\hline Sodium & percent & .005 & & & \\
\hline Strontium & $\mu \mathrm{g} / \mathrm{g}$ & $2^{.000}$ & & & \\
\hline
\end{tabular}


Table 3. Standard reference-material concentrations for inorganic-constituent analyses for tributaries to Lake Champlain

Ipercent, concentrations in weight percent, all other elements are in micrograms per gram $(\mathrm{mg} / \mathrm{g})$; values in parentheses are two standard deviations; SDO-1 had two laboratory results per sample; <, less than]

\begin{tabular}{|c|c|c|c|c|c|c|c|}
\hline \multirow{2}{*}{ Elements } & \multicolumn{3}{|c|}{ MAG-1 } & \multicolumn{4}{|c|}{ SDO-1 } \\
\hline & \multicolumn{2}{|c|}{ Standard results } & \multirow{2}{*}{$\frac{\text { Laboratory results }}{8.5}$} & \multicolumn{2}{|c|}{ Standard results } & \multicolumn{2}{|c|}{ Laboratory results } \\
\hline Aluminum (percent) & 8.70 & -- & & 6.50 & $(0.12)$ & 6.4 & 6.5 \\
\hline Arsenic & -- & -- & -- & 68.5 & $(8.6)$ & 68 & 67 \\
\hline Barium & $493(14$ & 40) & 470 & 397 & $(38)$. & 240 & 250 \\
\hline Calcium (percent) & 1.07 & -- & 1.1 & .75 & $(.03)$ & .78 & .78 \\
\hline Cerium & -- & -- & -- & 79.3 & $(7.8)$ & 72 & 73 \\
\hline Chromium & 121 & 22) & 120 & 66.4 & $(7.6)$ & 67 & 68 \\
\hline Cobalt & 17.6 & $(3.4)$ & 22 & 46.8 & $(5.3)$ & 48 & 48 \\
\hline Copper & 48.8 & $(5.6)$ & 30 & ${ }^{1} 60.2$ & $(9.6)$ & 56 & 55 \\
\hline Europium & -- & -- & -- & 1.6 & $(.22)$ & $<2$ & $<2$ \\
\hline Gallium & 20.9 & $(4.1)$ & 22 & 16.8 & $(1.8)$ & 15 & 15 \\
\hline Iron (percent) & 4.6 & -- & 4.8 & 6.53 & $(.15)$ & 6.4 & 6.5 \\
\hline Lanthanum & -- & -- & -- & 38.5 & $(4.4)$ & 34 & 34 \\
\hline Lead & -- & -- & -- & 127.9 & $(5.2)$ & 24 & 25 \\
\hline Magnesium (percent) & 1.79 & -- & 1.7 & .93 & .023 & .89 & $9 \quad .90$ \\
\hline Manganese & 774 & -- & 770 & 325 & $(40)$ & 310 & 320 \\
\hline Mercury & -- & - & - & 1.19 & $(.08)$ & .22 & .22 \\
\hline Molybdenum & -- & -- & -- & 134 & $(21)$. & 150 & 150 \\
\hline Neodymium & -- & -- & -- & 36.6 & $(3.3)$ & 32 & 34 \\
\hline Nickel & 50.7 & $(9.0)$ & 55 & 99.5 & $(9.9)$ & 94 & 96 \\
\hline Niobium & -- & -- & -- & 11.4 & $(1.2)$ & 9 & 8 \\
\hline Phosphorus (percent) & .14 & -- & .08 & .04 & $8(.003)$ & .05 & .05 \\
\hline Potassium (percent) & 2.99 & -- & 2.9 & 2.78 & $(.05)$ & 2.5 & 2.5 \\
\hline Scandium & 18.3 & $(2.1)$ & 17 & 13.2 & $(1.5)$ & 13 & 13 \\
\hline Sodium (percent) & 2.90 & -- & 2.7 & -- & -- & .29 & .29 \\
\hline Strontium & 158 & $(40.8)$ & 150 & 75.1 & (11) & 77 & 77 \\
\hline Tantalum & -- & -- & -- & ${ }^{1} 1.1$ & $(.13)$ & $<40$ & $<40$ \\
\hline Tin & -- & -- & -- & 13.7 & $(1.2)$ & $<5$ & $<5$ \\
\hline Uranium & -- & -- & -- & 48.8 & $(6.5)$ & $<100$ & $<100$ \\
\hline Vanadium & 132 & $(30.5)$ & 130 & 160 & $(21)$. & 150 & 160 \\
\hline Zinc & -- & - & -- & 64.1 & $(6.9)$ & 57 & 59 \\
\hline
\end{tabular}

${ }^{1}$ Values are less well established. 
Table 4. Results, in percent, from one-way, nested analysis of variance of ranked sedimentconstituent-concentration data for tributaries to Lake Champlain

[All values are in percent]

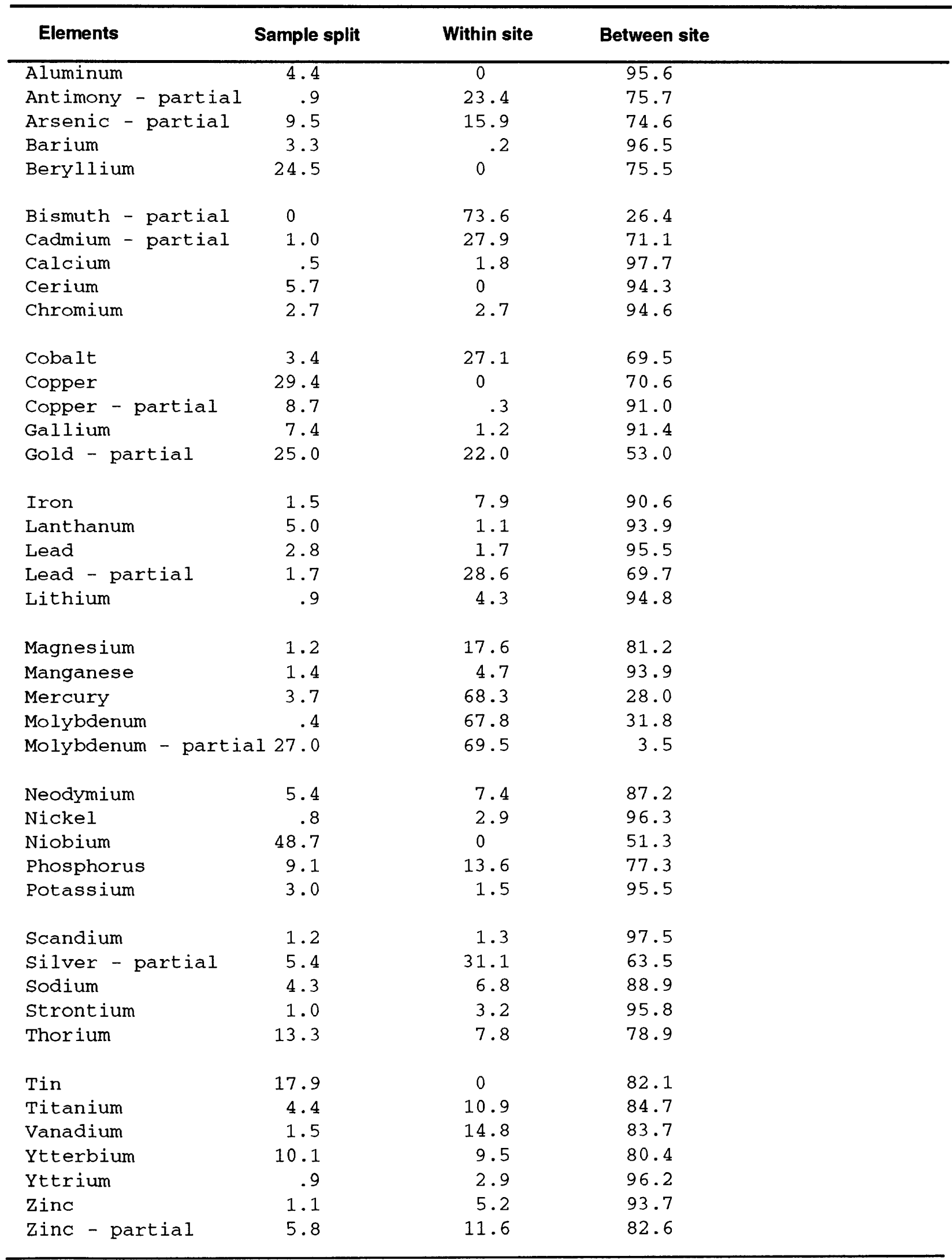


Table 5. Percentile distribution of element concentrations in 86 samples of fine-fraction streambed sediment for tributaries to Lake Champlain

[pct, percent; $\mu \mathrm{g} / \mathrm{g}$, micrograms per gram; $<$, less than reporting level given]

\begin{tabular}{|c|c|c|c|c|c|c|c|c|}
\hline \multirow{2}{*}{ Element } & \multirow{2}{*}{$\begin{array}{c}\text { Units } \\
\text { of } \\
\text { Concentration }\end{array}$} & \multirow{2}{*}{$\begin{array}{l}\text { Min- } \\
\text { imum }\end{array}$} & \multirow{2}{*}{$\begin{array}{l}\text { Max- } \\
\text { imum }\end{array}$} & \multicolumn{5}{|c|}{ Percent Distribution } \\
\hline & & & & 10 & 25 & 50 & 75 & 90 \\
\hline Aluminum & pet & 3.1 & 8.2 & 5.2 & 5.6 & 6.1 & 6.78 & 7.5 \\
\hline Antimony ${ }^{1}$ & $\mu \mathrm{g} / \mathrm{g}$ & $<.6$ & 4.2 & $<.6$ & $<.6$ & $<.6$ & $<.6$ & .8 \\
\hline Arsenic & $\mu \mathrm{g} / \mathrm{g}$ & $<10$ & 42 & $<10$ & $<10$ & $<10$ & $<10$ & $<10$ \\
\hline Arsenic $^{1}$ & $\mu \mathrm{g} / \mathrm{g}$ & $<.6$ & 45 & 1.4 & 2.2 & 3.4 & 4.8 & 7.1 \\
\hline Barium & $\mu \mathrm{g} / \mathrm{g}$ & 260 & 680 & 400 & 460 & 550 & 620 & 650 \\
\hline Beryllium & $\mu \mathrm{g} / \mathrm{g}$ & $<1$ & 3 & $<1$ & $<1$ & $<1$ & 2 & 2 \\
\hline Bismuth & $\mu \mathrm{g} / \mathrm{g}$ & $<10$ & $<10$ & $<10$ & $<10$ & $<10$ & $<10$ & $<10$ \\
\hline Bismuth $^{1}$ & $\mu \mathrm{g} / \mathrm{g}$ & $<.6$ & 1.1 & $<.6$ & $<.6$ & $<.6$ & $<.6$ & $<.6$ \\
\hline Cadmium & $\mu \mathrm{g} / \mathrm{g}$ & $<2$ & 3 & $<2$ & $<2$ & $<2$ & $<2$ & $<2$ \\
\hline Cadmium 1 & $\mu \mathrm{g} / \mathrm{g}$ & .08 & 2.4 & .12 & .16 & .23 & .35 & .62 \\
\hline Calcium & pct & .6 & 7.9 & 1.0 & 1.4 & 2 & 3 & 4.3 \\
\hline Cerium & $\mu \mathrm{g} / \mathrm{g}$ & 26 & 510 & 54 & 64 & 80 & 92 & 110 \\
\hline Chromium & $\mu \mathrm{g} / \mathrm{g}$ & 40 & 190 & 52 & 62 & 73 & 88 & 110 \\
\hline Cobalt & $\mu \mathrm{g} / \mathrm{g}$ & 6 & 44 & 10 & 12 & 15 & 18 & 23 \\
\hline Copper & $\mu \mathrm{g} / \mathrm{g}$ & 5 & 51 & 8.3 & 11 & 16 & 23 & 28 \\
\hline Copper ${ }^{1}$ & $\mu \mathrm{g} / \mathrm{g}$ & 4.0 & 47 & 8.4 & 12 & 18 & 24 & 30 \\
\hline Europium & $\mu \mathrm{g} / \mathrm{g}$ & $<2$ & 3 & $<2$ & $<2$ & $<2$ & $<2$ & $<2$ \\
\hline Gallium & $\mu \mathrm{g} / \mathrm{g}$ & 8 & 29 & 12 & 14 & 16 & 17 & 20 \\
\hline Gold & $\mu \mathrm{g} / \mathrm{g}$ & $<8$ & $<8$ & $<8$ & $<8$ & $<8$ & $<8$ & $<8$ \\
\hline Gold $^{1}$ & $\mu \mathrm{g} / \mathrm{g}$ & $<.1$ & $<.1$ & $<.1$ & $<.1$ & $<.1$ & $<.1$ & $<.1$ \\
\hline Holmium & $\mu \mathrm{g} / \mathrm{g}$ & $<4$ & 5 & $<4$ & $<4$ & $<4$ & $<4$ & $<4$ \\
\hline Iron & pct & 2.1 & 9.4 & 2.8 & 3.4 & 3.7 & 4.3 & 4.6 \\
\hline Lanthanum & $\mu \mathrm{g} / \mathrm{g}$ & 13 & 290 & 26 & 30 & 38 & 43 & 48 \\
\hline Lead & $\mu \mathrm{g} / \mathrm{g}$ & 9 & 280 & 11 & 14 & 16 & 21 & 37 \\
\hline Lead $^{1}$ & $\mu \mathrm{g} / \mathrm{g}$ & 4.9 & 340 & 8.5 & 11 & 16 & 23 & 37 \\
\hline Lithium & $\mu \mathrm{g} / \mathrm{g}$ & 9 & 50 & 14 & 19 & 27 & 34 & 45 \\
\hline Magnesium & pet & .53 & 3 & .79 & .95 & 1.1 & 1.4 & 1.5 \\
\hline Manganese & $\mu \mathrm{g} / \mathrm{g}$ & 310 & 7,000 & 630 & 800 & 1,200 & 1,600 & 2,400 \\
\hline Mercury & $\mu \mathrm{g} / \mathrm{g}$ & .03 & 3.7 & .073 & .11 & .20 & .34 & .47 \\
\hline Molybdenum & $\mu \mathrm{g} / \mathrm{g}$ & $<2$ & 4 & $<2$ & $<2$ & $<2$ & $<2$ & $<2$ \\
\hline Molybdenum $^{1}$ & $\mu \mathrm{g} / \mathrm{g}$ & .06 & 6.9 & .51 & .69 & .94 & 1.7 & 2.9 \\
\hline Neodymium & $\mu \mathrm{g} / \mathrm{g}$ & 12 & 190 & 27 & 32 & 37 & 41 & 46 \\
\hline Nickel & $\mu \mathrm{g} / \mathrm{g}$ & 13 & 110 & 21 & 27 & 33 & 41 & 57 \\
\hline Niobium & $\mu \mathrm{g} / \mathrm{g}$ & $<4$ & 19 & 7 & 9 & 10 & 12 & 12 \\
\hline Phosphorus & pct & .08 & .34 & .10 & .11 & .12 & .14 & .16 \\
\hline Potassium & pct & .87 & 3 & 1.5 & 1.7 & 2.1 & 2.3 & 2.5 \\
\hline Scandium & $\mu \mathrm{g} / \mathrm{g}$ & 5 & 15 & 7 & 9 & 11 & 12 & 14 \\
\hline Silver & $\mu \mathrm{g} / \mathrm{g}$ & $<2$ & 12 & $<2$ & $<2$ & $<2$ & $<2$ & $<2$ \\
\hline Silver $^{1}$ & $\mu \mathrm{g} / \mathrm{g}$ & $<.06$ & 17 & $<.06$ & $<.06$ & $<.06$ & $<.06$ & .11 \\
\hline Sodium & pct & .60 & 1.8 & .92 & 1.1 & 1.3 & 1.5 & 1.6 \\
\hline
\end{tabular}


Table 5. Percentile distribution of element concentrations in 86 samples of fine-fraction streambed sediment for tributaries to Lake Champlain--Continued

\begin{tabular}{|c|c|c|c|c|c|c|c|c|}
\hline \multirow{2}{*}{$\begin{array}{l}\text { Element } \\
\text { Strontium }\end{array}$} & \multirow{2}{*}{$\begin{array}{c}\begin{array}{c}\text { Units } \\
\text { of }\end{array} \\
\mu \mathrm{g} / \mathrm{g}\end{array}$} & \multirow{2}{*}{$\begin{array}{l}\begin{array}{l}\text { Min- } \\
\text { imum }\end{array} \\
83\end{array}$} & \multirow{2}{*}{$\begin{array}{l}\begin{array}{l}\text { Max- } \\
\text { imum }\end{array} \\
550\end{array}$} & \multicolumn{5}{|c|}{ Percent Distribution } \\
\hline & & & & 150 & 170 & 200 & 240 & 310 \\
\hline Tantalum & $\mu \mathrm{g} / \mathrm{g}$ & $<40$ & $<40$ & $<40$ & $<40$ & $<40$ & $<40$ & $<40$ \\
\hline Thorium & $\mu \mathrm{g} / \mathrm{g}$ & $<4$ & 30 & 5 & 6 & 7 & 8 & 10 \\
\hline Tin & $\mu \mathrm{g} / \mathrm{g}$ & $<5$ & 220 & $<5$ & $<5$ & $<5$ & $<5$ & 5.7 \\
\hline Titanium & pet & .15 & 1.6 & .41 & .47 & .54 & .66 & .75 \\
\hline Uranium & $\mu \mathrm{g} / \mathrm{g}$ & $<100$ & $<100$ & $<100$ & $<100$ & $<100$ & $<100$ & $<100$ \\
\hline Vanadium & $\mu \mathrm{g} / \mathrm{g}$ & 41 & 110 & 51 & 61 & 73 & 84 & 100 \\
\hline Ytterbium & $\mu \mathrm{g} / \mathrm{g}$ & 1 & 16 & 2 & 2 & 3 & 3 & 4 \\
\hline Yttrium & $\mu \mathrm{g} / \mathrm{g}$ & 12 & 200 & 26 & 28 & 30 & 33 & 45 \\
\hline Zinc & $\mu \mathrm{g} / \mathrm{g}$ & 43 & 420 & 61 & 75 & 90 & 110 & 160 \\
\hline Zinc ${ }^{1}$ & $\mu \mathrm{g} / \mathrm{g}$ & 25 & 360 & 50 & 73 & 84 & 120 & 160 \\
\hline
\end{tabular}

\footnotetext{
${ }^{1}$ Partial digestion was used.
} 
Table 6. Inorganic-constituent concentrations in fine-fraction streambed sediment for tributaries to Lake Champlain

[--, data not available or insufficient sample for analysis; <, less than reporting level given]

\begin{tabular}{|c|c|c|c|c|c|c|c|}
\hline $\begin{array}{c}\text { Site } \\
\text { number }^{1}\end{array}$ & Stream name & Latitude & Longltude & $\underset{\text { remarke }}{\text { Dedign }}$ & Date & $\begin{array}{l}\text { pH } \\
\text { (standard } \\
\text { unita) }\end{array}$ & $\begin{array}{c}\text { Specific } \\
\text { conductance } \\
\text { (mlcroslemens } \\
\text { per centimeter) }\end{array}$ \\
\hline 1 & Pike River & $45^{\circ} 04^{\prime} 40.3^{\prime \prime}$ & $73^{\circ} 05^{\prime} 19.0^{\prime \prime}$ & 1 & $7-21-92$ & 8.7 & 389 \\
\hline 1 & Pike River & $45^{\circ} 04^{\prime} 40.3^{\prime \prime}$ & $73^{\circ} 05^{\prime} 19.0^{\prime \prime}$ & 2 & $7-21-92$ & 8.7 & 389 \\
\hline 1 & Pike River & $45^{\circ} 04^{\prime} 40.3^{\prime \prime}$ & $73^{\circ} 05^{\prime} 19.0^{\prime \prime}$ & 2 & $7-21-92$ & 8.7 & 389 \\
\hline 4 & Rock River & $44^{\circ} 59^{\prime} 46.9^{\prime \prime}$ & $73^{\circ} 04^{\prime} 23.4^{\prime \prime}$ & 1 & $7-02-92$ & 7.3 & 363 \\
\hline 4 & Rock River & $44^{\circ} 59^{\prime} 46.9^{\prime \prime}$ & $73^{\circ} 04^{\prime} 23.4^{\prime \prime}$ & 2 & $7-02-92$ & 7.3 & 363 \\
\hline 4 & Rock River & $44^{\circ} 59^{\prime} 46.9^{\prime \prime}$ & $73^{\circ} 04^{\prime} 23.4^{\prime \prime}$ & 2 & $7-02-92$ & 7.3 & 363 \\
\hline 5 & Carman Brook & $44^{\circ} 58^{\prime} 38.1^{\prime \prime}$ & $73^{\circ} 05^{\prime} 01.8^{\prime \prime}$ & 1 & $6-17-92$ & 8.2 & 297 \\
\hline 6 & Mud Creek & $44^{\circ} 58^{\prime} 05.5^{\prime \prime}$ & $73^{\circ} 16^{\prime} 10.5^{\prime \prime}$ & 1 & $7-22-92$ & 7.0 & 434 \\
\hline 8 & Youngman Brook & $44^{\circ} 57^{\prime} 22.7^{\prime \prime}$ & $73^{\circ} 06^{\prime} 23.6^{\prime \prime}$ & 3 & $10-15-92$ & -- & -- \\
\hline 8 & Youngman Brook & $44^{\circ} 57^{\prime} 22.7^{\prime \prime}$ & $73^{\circ} 06^{\prime} 23.6^{\prime \prime}$ & 1 & $6-17-92$ & 7.7 & 149 \\
\hline 9 & Great Chazy River & $44^{\circ} 57^{\prime} 13.1^{\prime \prime}$ & $73^{\circ} 25^{\prime} 03.8^{\prime \prime}$ & 2 & $7-22-92$ & 7.2 & 198 \\
\hline 9 & Great Chazy River & $44^{\circ} 57^{\prime} 13.1^{\prime \prime}$ & $73^{\circ} 25^{\prime} 03^{\prime} .8^{\prime \prime}$ & 1 & $7-22-92$ & 7.2 & 198 \\
\hline 9 & Great Chazy River & $44^{\circ} 57^{\prime} 13.1^{\prime \prime}$ & $73^{\circ} 25^{\prime} 03.8^{\prime \prime}$ & 2 & $7-22-92$ & 7.2 & 198 \\
\hline 10 & Corbeau Creek & $44^{\circ} 56^{\prime} 27.2^{\prime \prime}$ & $73^{\circ} 24^{\prime} 36.0^{\prime \prime}$ & 1 & $7-22-92$ & 7.4 & 479 \\
\hline 11 & Missisquoi River & $44^{\circ} 55^{\prime} 53.8^{\prime \prime}$ & $73^{\circ} 07 \cdot 36.0^{\prime \prime}$ & 2 & $7-02-92$ & 7.5 & 150 \\
\hline 11 & Missisquoi River & $44^{\circ} 55^{\prime} 53.8^{\prime \prime}$ & $73^{\circ} 07^{\prime} 36.0^{\prime \prime}$ & 1 & $7-02-92$ & 7.5 & 150 \\
\hline 11 & Missisquoi River & $44^{\circ} 55^{\prime} 53.8^{\prime \prime}$ & $73^{\circ} 07^{\prime} 36.0^{\prime \prime}$ & 2 & $7-02-92$ & 7.5 & 150 \\
\hline 13 & Little Chazy River & $44^{\circ} 54^{\prime} 10.0^{\prime \prime}$ & $73^{\circ} 24^{\prime} 52.9^{\prime \prime}$ & 1 & $7-22-92$ & 8.8 & 256 \\
\hline 14 & Stephens Brook & $44^{\circ} 50^{\prime} 56.6^{\prime \prime}$ & $73^{\circ} 07^{\prime} 08.0^{\prime \prime}$ & 1 & $7-02-92$ & 8.2 & 704 \\
\hline 14 & Stephens Brook & $44^{\circ} 50^{\prime} 56.6^{\prime \prime}$ & $73^{\circ} 07^{\prime} 08.0^{\prime \prime}$ & 3 & $10-15-92$ & -- & -- \\
\hline 15 & Stephens Brook & $44^{\circ} 50^{\prime} 50.0^{\prime \prime}$ & $73^{\circ} 05^{\prime} 52.0^{\prime \prime}$ & 3 & $10-15-92$ & -- & -- \\
\hline 16 & Riley Brook & $44^{\circ} 48^{\prime} 28.0^{\prime \prime}$ & $73^{\circ} 24^{\prime} 03.0^{\prime \prime}$ & 1 & $7-23-92$ & 7.2 & 649 \\
\hline 18 & Mill River & $44^{\circ} 46^{\prime} 47.0^{\prime \prime}$ & $73^{\circ} 08^{\prime} 39.2^{\prime \prime}$ & 1 & $7-02-92$ & 8.5 & 479 \\
\hline 19 & Dead Creek & $44^{\circ} 45^{\prime} 31.4^{\prime \prime}$ & $73^{\circ} 26^{\prime} 51.5^{\prime \prime}$ & 1 & $7-23-92$ & 7.3 & 417 \\
\hline 20 & Ray Brook & $44^{\circ} 45^{\prime} 09.7^{\prime \prime}$ & $73^{\circ} 27^{\prime} 48.6^{\prime \prime}$ & 1 & $7-23-92$ & 7.7 & 507 \\
\hline 21 & Kennon Brook & $44^{\circ} 44^{\prime} 12.5^{\prime \prime}$ & $73^{\circ} 28^{\prime} 18.1^{\prime \prime}$ & 1 & $7-23-92$ & 8.1 & 387 \\
\hline 26 & Saranac River & $44^{\circ} 41^{\prime} 57.6^{\prime \prime}$ & $73^{\circ} 26^{\prime} 58.5^{\prime \prime}$ & 1 & $7-28-92$ & 7.2 & 93 \\
\hline 26 & Saranac River & $44^{\circ} 41^{\prime} 57.6^{\prime \prime}$ & $73^{\circ} 26^{\prime} 58.5^{\prime \prime}$ & 2 & $7-28-92$ & 7.2 & 93 \\
\hline 26 & Saranac River & $44^{\circ} 41^{\prime} 57.6^{\prime \prime}$ & $73^{\circ} 26^{\prime} 58.5^{\prime \prime}$ & 2 & $7-28-92$ & 7.2 & 93 \\
\hline 29 & stone Bridge Brook & $44^{\circ} 41^{\prime} 14.4^{\prime \prime}$ & $73^{\circ} 11 \cdot 22.6^{\prime \prime}$ & 1 & $7-06-92$ & 7.6 & 278 \\
\hline 30 & unnamed tributary & $44^{\circ} 39^{\prime} 12.1^{\prime \prime}$ & $73^{\circ} 26^{\prime} 41.0^{\prime \prime}$ & 1 & $7-29-92$ & 7.4 & 530 \\
\hline 31 & Trout Brook & $44^{\circ} 39^{\prime} 11.3^{\prime \prime}$ & $73^{\circ} 11^{\prime} 58.9^{\prime \prime}$ & 1 & $6-18-92$ & 8.2 & 307 \\
\hline 32 & Salmon River & $44^{\circ} 37^{\prime} 41.2^{\prime \prime}$ & $73^{\circ} 26^{\prime} 55.0^{\prime \prime}$ & 1 & $7-29-92$ & 7.2 & 192 \\
\hline 32 & Salmon River & $44^{\circ} 37^{\prime} 41.2^{\prime \prime}$ & $73^{\circ} 26^{\prime} 55.0^{\prime \prime}$ & 2 & $7-29-92$ & 7.2 & 192 \\
\hline 32 & Salmon River & $44^{\circ} 37^{\prime} 41.2^{\prime \prime}$ & $73^{\circ} 26^{\prime} 55.0^{\prime \prime}$ & 2 & $7-29-92$ & 7.2 & 192 \\
\hline 33 & Silver Stream & $44^{\circ} 37^{\prime} 14.0^{\prime \prime}$ & $73^{\circ} 26^{\prime} 52.8^{\prime \prime}$ & 1 & $7-23-92$ & 7.6 & 568 \\
\hline 34 & Lamoille River & $44^{\circ} 36^{\prime} 52.9^{\prime \prime}$ & $73^{\circ} 10^{\prime} 50.6^{\prime \prime}$ & 1 & $7-06-92$ & 6.7 & -- \\
\hline 36 & Little Ausable River & $44^{\circ} 34^{\prime} 58.2^{\prime \prime}$ & $73^{\circ} 27^{\prime} 29.6^{\prime \prime}$ & 1 & $7-29-92$ & 7.4 & 251 \\
\hline 37 & Allen Brook & $44^{\circ} 34^{\prime} 43.3^{\prime \prime}$ & $73^{\circ} 09^{\prime} 28.6^{\prime \prime}$ & 1 & $6-18-92$ & 8.1 & 517 \\
\hline 39 & Malletts Creek & $44^{\circ} 34^{\prime} 00.9^{\prime \prime}$ & $73^{\circ} 09^{\prime} 16.2^{\prime \prime}$ & 1 & $7-06-92$ & 8.0 & 313 \\
\hline
\end{tabular}




\begin{tabular}{|c|c|c|c|c|c|c|c|}
\hline $\begin{array}{c}\text { Site } \\
\text { number }^{1}\end{array}$ & Stream name & Latitude & Longitude & $\underset{\text { remarks }}{\text { Design }}$ & Date & $\begin{array}{c}\mathrm{pH} \\
\text { (standard } \\
\text { unlts) }\end{array}$ & $\begin{array}{c}\text { Specific } \\
\text { conductance } \\
\text { (microslemens } \\
\text { per centimeter) }\end{array}$ \\
\hline 41 & Indian Brook & $44^{\circ} 33^{\prime} 33.8^{\prime \prime}$ & $73^{\circ} 10^{\prime} 50.8^{\prime \prime}$ & 1 & $7-06-92$ & 7.6 & 493 \\
\hline 42 & Pond Brook & $44^{\circ} 33^{\prime} 30.5^{\prime \prime}$ & $73^{\circ} 09^{\prime} 52.9^{\prime \prime}$ & 1 & $6-19-92$ & 7.6 & 401 \\
\hline 43 & Ausable River & $44^{\circ} 33^{\prime} 19.5^{\prime \prime}$ & $73^{\circ} 27^{\prime} 26.4^{\prime \prime}$ & 1 & $7-27-92$ & 7.8 & 97 \\
\hline 43 & Ausable River & $44^{\circ} 33^{\prime} 19.5^{\prime \prime}$ & $73^{\circ} 27^{\prime} 26.4^{\prime \prime}$ & 2 & $7-27-92$ & 7.8 & 97 \\
\hline 43 & Ausable River & $44^{\circ} 33^{\prime} 19.5^{\prime \prime}$ & $73^{\circ} 27^{\prime} 26.4^{\prime \prime}$ & 2 & $7-27-92$ & 7.8 & 97 \\
\hline 44 & Winooski River & $44^{\circ} 32^{\prime} 20.7^{\prime \prime}$ & $73^{\circ} 15^{\prime} 56.0^{\prime \prime}$ & 1 & $7-01-92$ & 8.0 & 246 \\
\hline 46 & Winooski River & $44^{\circ} 29^{\prime} 20.2^{\prime \prime}$ & $73^{\circ} 11^{\prime} 03.1^{\prime \prime}$ & 1 & $7-06-92$ & 8.0 & 236 \\
\hline 47 & Little Trout Brook & $44^{\circ} 28^{\prime} 49.0^{\prime \prime}$ & $73^{\circ} 25^{\prime} 06.7^{\prime \prime}$ & 1 & $7-27-92$ & 8.2 & 369 \\
\hline 48 & Potash Brook & $44^{\circ} 26^{\prime} 22.6^{\prime \prime}$ & $73^{\circ} 13^{\prime} 10.7^{\prime \prime}$ & 1 & $6-19-92$ & 8.1 & 910 \\
\hline 49 & unnamed tributary & $44^{\circ} 25^{\prime} 37.2^{\prime \prime}$ & $73^{\circ} 24^{\prime} 51.0^{\prime \prime}$ & 1 & $7-27-92$ & 7.4 & 166 \\
\hline 51 & Munroe Brook & $44^{\circ} 24^{\prime} 16.1^{\prime \prime}$ & $73^{\circ} 13^{\prime} 03.0^{\prime \prime}$ & 1 & $6-26-92$ & 7.6 & 631 \\
\hline 52 & Laplatte River & $44^{\circ} 23^{\prime} 05.0^{\prime \prime}$ & $73^{\circ} 13^{\prime} 26.8^{\prime \prime}$ & 1 & $7-07-92$ & 7.7 & 446 \\
\hline 53 & Bouquet River & $44^{\circ} 22^{\prime} 10.8^{\prime \prime}$ & $73^{\circ} 23^{\prime} 28.0^{\prime \prime}$ & 2 & $7-27-92$ & 8.1 & 149 \\
\hline 53 & Bouquet River & $44^{\circ} 22^{\prime} 10.8^{\prime \prime}$ & $73^{\circ} 23^{\prime} 28.0^{\prime \prime}$ & 1 & $7-27-92$ & 8.1 & 149 \\
\hline 53 & Bouquet River & $44^{\circ} 22^{\prime} 10.8^{\prime \prime}$ & $73^{\circ} 23^{\prime} 28.0^{\prime \prime}$ & 2 & $7-27-92$ & 8.1 & 149 \\
\hline 55 & Holmes Creek & $44^{\circ} 19^{\prime} 50.6^{\prime \prime}$ & $73^{\circ} 16^{\prime} 34.5^{\prime \prime}$ & 1 & $7-07-92$ & 7.7 & 385 \\
\hline 56 & Thorp Brook & $44^{\circ} 16^{\prime} 27.2^{\prime \prime}$ & $73^{\circ} 15^{\prime} 20.5^{\prime \prime}$ & 1 & $7-07-92$ & 8.2 & 508 \\
\hline 57 & Lewis Creek & $44^{\circ} 14^{\prime} 45.9^{\prime \prime}$ & $73^{\circ} 14^{\prime} 44.5^{\prime \prime}$ & 1 & $7-07-92$ & 8.8 & 248 \\
\hline 59 & Little otter Creek & $44^{\circ} 11^{\prime} 53.8^{\prime \prime}$ & $73^{\circ} 14^{\prime} 46.2^{\prime \prime}$ & 1 & $7-07-92$ & 8.7 & 346 \\
\hline 61 & Hoisington Brook & $44^{\circ} 11^{\prime} 00.0^{\prime \prime}$ & $73^{\circ} 26^{\prime} 06.7^{\prime \prime}$ & 1 & $7-10-92$ & 7.5 & 223 \\
\hline 62 & otter Creek & $44^{\circ} 09^{\prime} 27.0^{\prime \prime}$ & $73^{\circ} 17^{\prime} 01.0^{\prime \prime}$ & 1 & $7-08-92$ & 8.5 & 230 \\
\hline 63 & Stacy Brook & $44^{\circ} 08^{\prime} 33.6^{\prime \prime}$ & $73^{\circ} 25^{\prime} 51.8^{\prime \prime}$ & 1 & $7-10-92$ & 7.6 & 180 \\
\hline 64 & Mullen Brook & $44^{\circ} 06^{\prime} 38.0^{\prime \prime}$ & $73^{\circ} 27^{\prime} 04.0^{\prime \prime}$ & 3 & $10-21-92$ & -- & -- \\
\hline 65 & unnamed tributary & $44^{\circ} 06^{\prime} 06.0^{\prime \prime}$ & $73^{\circ} 27^{\prime} 03.0^{\prime \prime}$ & 3 & $10-21-92$ & -- & -- \\
\hline 66 & Mullen Brook & $44^{\circ} 06^{\prime} 04.7^{\prime \prime}$ & $73^{\circ} 27^{\prime} 00.0^{\prime \prime}$ & 1 & $7-09-92$ & 7.6 & 155 \\
\hline 66 & Mullen Brook & $44^{\circ} 06^{\prime} 04.7^{\prime \prime}$ & $73^{\circ} 27^{\prime} 00.0^{\prime \prime}$ & 3 & $10-21-92$ & -- & -- \\
\hline 67 & Mil1 Brook & $44^{\circ} 03^{\prime} 10.1^{\prime \prime}$ & $73^{\circ} 27^{\prime} 22.6^{\prime \prime}$ & 1 & $7-09-92$ & 8.2 & 149 \\
\hline 67 & Mill Brook & $44^{\circ} 03^{\prime} 10.1^{\prime \prime}$ & $73^{\circ} 27^{\prime} 22.6^{\prime \prime}$ & 3 & $10-21-92$ & -- & -- \\
\hline 68 & Mil1 Brook & $44^{\circ} 02^{\prime} 58.0^{\prime \prime}$ & $73^{\circ} 28^{\prime} 08.0^{\prime \prime}$ & 3 & $10-21-92$ & -- & -- \\
\hline 69 & McKenzie Brook & $44^{\circ} 02^{\prime} 08.3^{\prime \prime}$ & $73^{\circ} 27^{\prime} 49.2^{\prime \prime}$ & 1 & $7-10-92$ & 8.6 & 281 \\
\hline 70 & E. Branch Dead Creek & $44^{\circ} 01^{\prime} 41.8^{\prime \prime}$ & $73^{\circ} 19^{\prime} 15.6^{\prime \prime}$ & 1 & $7-08-92$ & 8.4 & 885 \\
\hline 71 & W. Branch Dead Creek & $44^{\circ} 01^{\prime} 32.5^{\prime \prime}$ & $73^{\circ} 20^{\prime} 53.6^{\prime \prime}$ & 1 & $7-08-92$ & 9.3 & 816 \\
\hline 72 & Grove Brook & $44^{\circ} 00^{\prime} 39.0^{\prime \prime}$ & $73^{\circ} 27^{\prime} 24.9^{\prime \prime}$ & 1 & $7-10-92$ & 8.0 & 237 \\
\hline 73 & Putnam Creek & $43^{\circ} 57^{\prime} 21.2^{\prime \prime}$ & $73^{\circ} 25^{\prime} 59.4^{\prime \prime}$ & 1 & $7-09-92$ & 8.3 & 297 \\
\hline 75 & Grant Brook & $43^{\circ} 54^{\prime} 24.2^{\prime \prime}$ & $73^{\circ} 25^{\prime} 25.7^{\prime \prime}$ & 1 & $7-23-92$ & 8.2 & 490 \\
\hline 76 & Five Mile Creek & $43^{\circ} 53^{\prime} 43.1^{\prime \prime}$ & $73^{\circ} 24^{\prime} 17.1^{\prime \prime}$ & 1 & $7-23-92$ & 7.8 & 363 \\
\hline 77 & unnamed tributary & $43^{\circ} 51^{\prime} 00.0^{\prime \prime}$ & $73^{\circ} 21^{\prime} 50.8^{\prime \prime}$ & 1 & $7-16-92$ & 7.5 & 1095 \\
\hline 78 & Ticonderoga Creek & $43^{\circ} 50^{\prime} 49.3^{\prime \prime}$ & $73^{\circ} 24^{\prime} 37.9^{\prime \prime}$ & 1 & $7-24-92$ & 8.0 & 124 \\
\hline 78 & Ticonderoga Creek & $43^{\circ} 50^{\prime} 49.3^{\prime \prime}$ & $73^{\circ} 24^{\prime} 37.9^{\prime \prime}$ & 2 & $7-24-92$ & 8.0 & 124 \\
\hline 78 & Ticonderoga Creek & $43^{\circ} 50^{\prime} 49.3^{\prime \prime}$ & $73^{\circ} 24^{\prime} 37.9^{\prime \prime}$ & 2 & $7-24-92$ & 8.0 & 124 \\
\hline 80 & East Creek & $43^{\circ} 48^{\prime} 13.5^{\prime \prime}$ & $73^{\circ} 20^{\prime} 16.2^{\prime \prime}$ & 1 & $7-16-92$ & 7.7 & 513 \\
\hline 81 & Charter Brook & $43^{\circ} 48^{\prime} 04.6^{\prime \prime}$ & $73^{\circ} 23^{\prime} 42.8^{\prime \prime}$ & 1 & $7-09-92$ & 8.1 & 266 \\
\hline 82 & South Brook & $43^{\circ} 46^{\prime} 19.2^{\prime \prime}$ & $73^{\circ} 23^{\prime} 21.0^{\prime \prime}$ & 1 & $7-09-92$ & 7.6 & 469 \\
\hline 83 & Mill Brook & $43^{\circ} 43^{\prime} 59.2^{\prime \prime}$ & $73^{\circ} 23^{\prime} 35.7^{\prime \prime}$ & 1 & $7-09-92$ & 8.0 & 311 \\
\hline 84 & unnamed tributary & $43^{\circ} 43^{\prime} 28.3^{\prime \prime}$ & $73^{\circ} 21^{\prime} 52.5^{\prime \prime}$ & 1 & $7-14-92$ & 8.0 & 381 \\
\hline
\end{tabular}


Table 6. Inorganic-constituent concentrations in fine-fraction streambed sediment for tributaries to Lake Champlain--Continued

\begin{tabular}{|c|c|c|c|c|c|c|c|}
\hline $\begin{array}{l}\text { Site } \\
\text { number } 1\end{array}$ & Stream name & Latitude & Longltude & $\begin{array}{l}\text { Design } \\
\text { remarks } 2\end{array}$ & Date & $\begin{array}{c}\text { pH } \\
\text { (standard } \\
\text { unlts) }\end{array}$ & $\begin{array}{c}\text { Specific } \\
\text { conductance } \\
\text { (microslemens } \\
\text { per centimeter) }\end{array}$ \\
\hline 85 & unnamed tributary & $43^{\circ} 40^{\prime} 04.6^{\prime \prime}$ & $73^{\circ} 25^{\prime} 04.5^{\prime \prime}$ & 3 & $10-21-92$ & -- & -- \\
\hline 85 & unnamed tributary & $43^{\circ} 40^{\prime} 04.6^{\prime \prime}$ & $73^{\circ} 25^{\prime} 04.5^{\prime \prime}$ & 1 & $7-24-92$ & 7.6 & 371 \\
\hline 86 & Horton Brook & $43^{\circ} 38^{\prime} 55.9^{\prime \prime}$ & $73^{\circ} 23^{\prime} 35.9^{\prime \prime}$ & 1 & $7-16-92$ & 8.2 & 687 \\
\hline 87 & unnamed tributary & $43^{\circ} 38^{\prime} 15.6^{\prime \prime}$ & $73^{\circ} 26^{\prime} 23.1^{\prime \prime}$ & 1 & $7-24-92$ & 8.3 & 276 \\
\hline 88 & Hubbardton River & $43^{\circ} 37^{\prime} 37.5^{\prime \prime}$ & $73^{\circ} 20^{\prime} 33.2^{\prime \prime}$ & 1 & $7-16-92$ & 8.4 & 350 \\
\hline 89 & Poultney River & $43^{\circ} 37^{\prime} 33.9^{\prime \prime}$ & $73^{\circ} 20^{\prime} 35.5^{\prime \prime}$ & 1 & $7-16-92$ & 7.7 & 215 \\
\hline 89 & Poultney River & $43^{\circ} 37^{\prime} 33.9^{\prime \prime}$ & $73^{\circ} 20^{\prime} 35.5^{\prime \prime}$ & 2 & $7-16-92$ & 7.7 & 215 \\
\hline 89 & Poultney River & $43^{\circ} 37^{\prime} 33.9^{\prime \prime}$ & $73^{\circ} 20^{\prime} 35.5^{\prime \prime}$ & 2 & $7-16-92$ & 7.7 & 215 \\
\hline 90 & Coggman Creek & $43^{\circ} 37^{\prime} 32.9^{\prime \prime}$ & $73^{\circ} 22^{\prime} 16.8^{\prime \prime}$ & 1 & $7-16-92$ & 7.4 & 421 \\
\hline 91 & Pine Lake Brook & $43^{\circ} 37^{\prime} 07.1^{\prime \prime}$ & $73^{\circ} 26^{\prime} 23.9^{\prime \prime}$ & 1 & $7-24-92$ & 6.8 & 94 \\
\hline 92 & Pike Brook & $43^{\circ} 33^{\prime} 02.4^{\prime \prime}$ & $73^{\circ} 27^{\prime} 55.1^{\prime \prime}$ & 1 & $7-17-92$ & 7.5 & 83 \\
\hline 93 & Mud Brook & $43^{\circ} 32^{\prime} 42.5^{\prime \prime}$ & $73^{\circ} 23^{\prime} 41.7^{\prime \prime}$ & 1 & $7-15-92$ & 7.5 & 519 \\
\hline 94 & Greenland Brook & $43^{\circ} 32^{\prime} 06.4^{\prime \prime}$ & $73^{\circ} 30^{\prime} 22.5^{\prime \prime}$ & 1 & $7-17-92$ & 8.8 & 38 \\
\hline 95 & Metawee River & $43^{\circ} 31^{\prime} 46.6^{\prime \prime}$ & $73^{\circ} 23^{\prime} 25.8^{\prime \prime}$ & 1 & $7-15-92$ & 7.9 & 230 \\
\hline 96 & Spectacle Brook & $43^{\circ} 31^{\prime} 23.3^{\prime \prime}$ & $73^{\circ} 30^{\prime} 35.6^{\prime \prime}$ & 1 & $7-17-92$ & 7.0 & 53 \\
\hline 97 & Mount Hope Brook & $43^{\circ} 31^{\prime} 19.8^{\prime \prime}$ & $73^{\circ} 30^{\prime} 29.5^{\prime \prime}$ & 1 & $7-17-92$ & 8.2 & 86 \\
\hline
\end{tabular}


Table 6. Inorganic-constituent concentrations in fine-fraction streambed sediment for tributaries to Lake Champlain--Continued

\begin{tabular}{|c|c|c|c|c|c|c|c|c|c|}
\hline $\begin{array}{l}\text { Site } \\
\text { number }\end{array}$ & $\underset{\text { remarks }}{\text { Design }}$ & $\begin{array}{l}\text { Aluminum } \\
\text { (weight } \\
\text { percent) }\end{array}$ & $\begin{array}{c}\text { Antimony } \\
\text {-partiai } \\
\text { (micro- } \\
\text { grams } \\
\text { per gram) }\end{array}$ & $\begin{array}{c}\text { Arsenic } \\
\text { (micro- } \\
\text { grams } \\
\text { per gram) }\end{array}$ & $\begin{array}{c}\text { Arsenic } \\
\text { - partiai } \\
\text { (micro- } \\
\text { grams } \\
\text { per gram) }\end{array}$ & $\begin{array}{l}\text { Barium } \\
\text { (micro- } \\
\text { grame } \\
\text { per gram) }\end{array}$ & $\begin{array}{c}\text { Beryliium } \\
\text { (micro- } \\
\text { grams } \\
\text { per gram) }\end{array}$ & $\begin{array}{l}\text { Biemuth } \\
\text { (micro- } \\
\text { grams } \\
\text { per gram) }\end{array}$ & $\begin{array}{l}\text { Bismuth } \\
\text { - partial } \\
\text { (micro- } \\
\text { grams } \\
\text { per gram) }\end{array}$ \\
\hline 1 & 1 & 6.9 & $<0.6$ & $<10$ & 1.6 & 670 & 2 & $<10$ & $<0.6$ \\
\hline 1 & 2 & 6.6 & $<.6$ & $<10$ & 1.3 & 650 & $<1$ & $<10$ & $<.6$ \\
\hline 1 & 2 & 6.7 & $<.6$ & $<10$ & 1.3 & 660 & 2 & $<10$ & $<.6$ \\
\hline 4 & 1 & 7.5 & $<.6$ & $<10$ & 2.6 & 640 & 2 & $<10$ & $<.6$ \\
\hline 4 & 2 & 7.4 & $<.6$ & $<10$ & 2.7 & 630 & 2 & $<10$ & $<.6$ \\
\hline 4 & 2 & 7.7 & $<.6$ & $<10$ & 2.9 & 640 & 2 & $<10$ & $<.6$ \\
\hline 5 & 1 & 5.7 & $<.6$ & $<10$ & 4.4 & 450 & $<1$ & $<10$ & $<.6$ \\
\hline 6 & 1 & 3.1 & 4.0 & $<10$ & 2.2 & 260 & $<1$ & $<10$ & $<.6$ \\
\hline 8 & 3 & 5.6 & -- & 19 & -- & 480 & $<1$ & $<10$ & -- \\
\hline 8 & 1 & 5.6 & 4.2 & 15 & 19 & 490 & $<1$ & $<10$ & $<.6$ \\
\hline 9 & 2 & 5.8 & $<.6$ & $<10$ & 1.1 & 630 & $<1$ & $<10$ & $<.6$ \\
\hline 9 & 1 & 5.6 & $<.6$ & $<10$ & .8 & 630 & $<1$ & $<10$ & $<.6$ \\
\hline 9 & 2 & 5.8 & $<.6$ & $<10$ & 1.0 & 640 & $<1$ & $<10$ & $<.6$ \\
\hline 10 & 1 & 5.4 & $<.6$ & $<10$ & 1.6 & 650 & $<1$ & $<10$ & $<.6$ \\
\hline 11 & 2 & 6.4 & $<.6$ & $<10$ & 2.0 & 490 & $<1$ & $<10$ & $<.6$ \\
\hline 11 & 1 & 6.3 & $<.6$ & $<10$ & 3.4 & 500 & $<1$ & $<10$ & $<.6$ \\
\hline 11 & 2 & 6.0 & $<.6$ & $<10$ & 2.7 & 470 & $<1$ & $<10$ & $<.6$ \\
\hline 13 & 1 & 5.3 & $<.6$ & $<10$ & 1.1 & 620 & $<1$ & $<10$ & $<.6$ \\
\hline 14 & 1 & 6.5 & .9 & $<10$ & 7.7 & 580 & 2 & $<10$ & 1.1 \\
\hline 14 & 3 & 6.5 & -- & $<10$ & -- & 590 & 2 & $<10$ & -- \\
\hline 15 & 3 & 6.8 & -- & 12 & -- & 580 & 2 & $<10$ & -- \\
\hline 16 & 1 & 5.8 & $<.6$ & $<10$ & $<.6$ & 650 & $<1$ & $<10$ & $<.6$ \\
\hline 18 & 1 & 6.3 & $<.6$ & $<10$ & 4.2 & 560 & $<1$ & $<10$ & $<.6$ \\
\hline 19 & 1 & 6.1 & $<.6$ & $<10$ & 1.5 & 620 & $<1$ & $<10$ & $<.6$ \\
\hline 20 & 1 & 5.5 & $<.6$ & $<10$ & 2.0 & 620 & $<1$ & $<10$ & $<.6$ \\
\hline 21 & 1 & 5.2 & $<.6$ & $<10$ & 4.5 & 680 & $<1$ & $<10$ & $<.6$ \\
\hline 26 & 1 & 4.9 & 1.2 & $<10$ & 3.2 & 610 & $<1$ & $<10$ & $<.6$ \\
\hline 26 & 2 & 5.0 & 1.4 & $<10$ & 3.5 & 590 & $<1$ & $<10$ & $<.6$ \\
\hline 26 & 2 & 4.8 & 1.6 & $<10$ & 3.1 & 570 & $<1$ & $<10$ & $<.6$ \\
\hline 29 & 1 & 5.4 & $<.6$ & $<10$ & 4.7 & 400 & $<1$ & $<10$ & $<.6$ \\
\hline 30 & 1 & 5.6 & $<.6$ & $<10$ & 5.4 & 630 & $<1$ & $<10$ & $<.6$ \\
\hline 31 & 1 & 6.2 & $<.6$ & $<10$ & 7.3 & 450 & $<1$ & $<10$ & $<.6$ \\
\hline 32 & 1 & 4.8 & $<.6$ & $<10$ & 1.6 & 560 & $<1$ & $<10$ & $<.6$ \\
\hline 32 & 2 & 4.6 & -- & $<10$ & -- & 560 & $<1$ & $<10$ & -- \\
\hline 32 & 2 & 4.5 & -- & $<10$ & $\cdots$ & 550 & $<1$ & $<10$ & -- \\
\hline 33 & 1 & 5.5 & $<.6$ & $<10$ & 2.7 & 570 & $<1$ & $<10$ & $<.6$ \\
\hline 34 & 1 & 7.3 & $<.6$ & 17 & 18 & 590 & 2 & $<10$ & $<.6$ \\
\hline 36 & 1 & 4.8 & $<.6$ & $<10$ & 2.3 & 560 & $<1$ & $<10$ & $<.6$ \\
\hline 37 & 1 & 6.1 & $<.6$ & $<10$ & 5.5 & 440 & $<1$ & $<10$ & $<.6$ \\
\hline 39 & 1 & 6.7 & $<.6$ & $<10$ & 4.4 & 550 & 2 & $<10$ & $<.6$ \\
\hline 41 & 1 & 5.9 & $<.6$ & 11 & 6.5 & 400 & $<1$ & $<10$ & $<.6$ \\
\hline 42 & 1 & 7.1 & $<.6$ & $<10$ & 8.2 & 540 & 2 & $<10$ & $<.6$ \\
\hline 43 & 1 & 5.6 & $<.6$ & $<10$ & 2.0 & 490 & $<1$ & $<10$ & $<.6$ \\
\hline 43 & 2 & 5.7 & -- & $<10$ & -- & 460 & $<1$ & $<10$ & -- \\
\hline
\end{tabular}


Table 6. Inorganic-constituent concentrations in fine-fraction streambed sediment for tributaries to Lake Champlain-Continued

\begin{tabular}{|c|c|c|c|c|c|c|c|c|c|}
\hline $\begin{array}{c}\text { Site } \\
\text { number }\end{array}$ & $\underset{\text { remarks }}{\text { Design }}$ & $\begin{array}{c}\text { Aluminum } \\
\text { (weight } \\
\text { percent) }\end{array}$ & $\begin{array}{c}\text { Antimony } \\
\text {-partlal } \\
\text { (micro- } \\
\text { grams } \\
\text { per gram) }\end{array}$ & $\begin{array}{l}\text { Arsenlc } \\
\text { (micro- } \\
\text { grams } \\
\text { per gram) }\end{array}$ & $\begin{array}{l}\text { Arsenic } \\
\text { - partlal } \\
\text { (micro- } \\
\text { grams } \\
\text { per gram) }\end{array}$ & $\begin{array}{l}\text { Barlum } \\
\text { (mloro- } \\
\text { grams } \\
\text { per gram) }\end{array}$ & $\begin{array}{c}\text { Beryllium } \\
\text { (micro- } \\
\text { grams } \\
\text { per gram) }\end{array}$ & $\begin{array}{l}\text { Blemuth } \\
\text { (micro- } \\
\text { grams } \\
\text { per gram) }\end{array}$ & $\begin{array}{l}\text { Blemuth } \\
\text { - partial } \\
\text { (micro- } \\
\text { grams } \\
\text { per gram) }\end{array}$ \\
\hline 43 & 2 & 5.3 & -- & $<10$ & -- & 420 & $<1$ & $<10$ & -- \\
\hline 44 & 1 & 6.0 & $<.6$ & $<10$ & 4.8 & 380 & 2 & $<10$ & $<.6$ \\
\hline 46 & 1 & 6.5 & $<.6$ & $<10$ & 5.8 & 440 & 2 & $<10$ & $<.6$ \\
\hline 47 & 1 & 5.0 & $<.6$ & $<10$ & $<.6$ & 590 & $<1$ & $<10$ & $<.6$ \\
\hline 48 & 1 & 5.9 & $<.6$ & $<10$ & 4.4 & 460 & $<1$ & $<10$ & $<.6$ \\
\hline 49 & 1 & 5.4 & $<.6$ & $<10$ & .6 & 580 & $<1$ & $<10$ & $<.6$ \\
\hline 51 & 1 & 6.0 & $<.6$ & $<10$ & 3.6 & 540 & $<1$ & $<10$ & $<.6$ \\
\hline 52 & 1 & 6.4 & $<.6$ & $<10$ & 3.2 & 570 & $<1$ & $<10$ & $<.6$ \\
\hline 53 & 2 & 6.9 & $<.6$ & $<10$ & 1.0 & 430 & $<1$ & $<10$ & $<.6$ \\
\hline 53 & 1 & 7.0 & $<.6$ & $<10$ & $<.6$ & 410 & $<1$ & $<10$ & $<.6$ \\
\hline 53 & 2 & 6.6 & $<.6$ & $<10$ & 1.7 & 410 & $<1$ & $<10$ & $<.6$ \\
\hline 55 & 1 & 8.2 & .7 & $<10$ & 3.8 & 670 & 2 & $<10$ & $<.6$ \\
\hline 56 & 1 & 7.3 & $<.6$ & $<10$ & 4.2 & 620 & 2 & $<10$ & $<.6$ \\
\hline 57 & 1 & 6.1 & $<.6$ & $<10$ & 4.3 & 430 & $<1$ & $<10$ & $<.6$ \\
\hline 59 & 1 & 6.5 & .7 & $<10$ & 3.9 & 560 & 2 & $<10$ & $<.6$ \\
\hline 61 & 1 & 6.8 & $<.6$ & $<10$ & 1.6 & 480 & $<1$ & $<10$ & $<.6$ \\
\hline 62 & 1 & 6.6 & $<.6$ & $<10$ & 2.3 & 520 & 2 & $<10$ & $<.6$ \\
\hline 63 & 1 & 7.1 & $<.6$ & $<10$ & 1.8 & 490 & $<1$ & $<10$ & $<.6$ \\
\hline 64 & 3 & 6.7 & -- & $<10$ & -- & 330 & $<1$ & $<10$ & -- \\
\hline 65 & 3 & 6.9 & -- & $<10$ & -- & 420 & $<1$ & $<10$ & -- \\
\hline 66 & 1 & 6.7 & $<.6$ & $<10$ & 3.1 & 410 & $<1$ & $<10$ & $<.6$ \\
\hline 66 & 3 & 6.4 & -- & $<10$ & -- & 400 & $<1$ & $<10$ & -- \\
\hline 67 & 1 & 5.8 & 1.2 & 42 & 45 & 330 & 3 & $<10$ & $<.6$ \\
\hline 67 & 3 & 5.8 & -- & 38 & -- & 350 & 3 & $<10$ & -- \\
\hline 68 & 3 & 6.0 & -- & 41 & -- & 340 & 3 & $<10$ & -- \\
\hline 69 & 1 & 5.6 & $<.6$ & $<10$ & 7.3 & 430 & $<1$ & $<10$ & $<.6$ \\
\hline 70 & 1 & 8.1 & $<.6$ & $<10$ & 4.4 & 640 & 2 & $<10$ & .7 \\
\hline 71 & 1 & 7.8 & $<.6$ & $<10$ & 3.8 & 620 & 2 & $<10$ & $<.6$ \\
\hline 72 & 1 & 5.2 & $<.6$ & $<10$ & 6.3 & 380 & $<1$ & $<10$ & $<.6$ \\
\hline 73 & 1 & 5.0 & $<.6$ & $<10$ & 3.3 & 470 & $<1$ & $<10$ & $<.6$ \\
\hline 75 & 1 & 6.4 & $<.6$ & $<10$ & 4.0 & 540 & $<1$ & $<10$ & $<.6$ \\
\hline 76 & 1 & 6.4 & $<.6$ & $<10$ & 3.8 & 550 & $<1$ & $<10$ & $<.6$ \\
\hline 77 & 1 & 7.3 & $<.6$ & $<10$ & 2.8 & 670 & 2 & $<10$ & $<.6$ \\
\hline 78 & 1 & 6.1 & 1.2 & $<10$ & 4.0 & 530 & 2 & $<10$ & $<.6$ \\
\hline 78 & 2 & 6.0 & 1.5 & $<10$ & 4.3 & 530 & 2 & $<10$ & $<.6$ \\
\hline 78 & 2 & 6.0 & 1.6 & $<10$ & 4.2 & 520 & 2 & $<10$ & $<.6$ \\
\hline 80 & 1 & 6.8 & $<.6$ & $<10$ & 2.5 & 570 & 2 & $<10$ & $<.6$ \\
\hline 81 & 1 & 7.6 & $<.6$ & $<10$ & 6.6 & 650 & 2 & $<10$ & $<.6$ \\
\hline 82 & 1 & 8.1 & $<.6$ & $<10$ & 4.7 & 620 & 2 & $<10$ & $<.6$ \\
\hline 83 & 1 & 6.1 & $<.6$ & $<10$ & 4.6 & 570 & 2 & $<10$ & $<.6$ \\
\hline 84 & 1 & 6.5 & $<.6$ & $<10$ & 3.4 & 590 & 2 & $<10$ & $<.6$ \\
\hline 85 & 3 & 5.1 & -- & $<10$ & -- & 470 & $<1$ & $<10$ & -- \\
\hline 85 & 1 & 5.2 & $<.6$ & $<10$ & 1.4 & 460 & $<1$ & $<10$ & $<.6$ \\
\hline 86 & 1 & 7.6 & $<.6$ & $<10$ & 2.8 & 640 & 2 & $<10$ & $<.6$ \\
\hline
\end{tabular}


Table 6. Inorganic-constituent concentrations in fine-fraction streambed sediment for tributaries to Lake Champlain--Continued

\begin{tabular}{|c|c|c|c|c|c|c|c|c|c|}
\hline $\begin{array}{l}\text { Slte } \\
\text { number } 1\end{array}$ & remarks $^{\text {Design }}$ & $\begin{array}{c}\text { Aluminum } \\
\text { (welght } \\
\text { percent) }\end{array}$ & $\begin{array}{c}\text { Antimony } \\
\text {-partlal } \\
\text { (micro- } \\
\text { grams } \\
\text { per gram) }\end{array}$ & $\begin{array}{c}\text { Arsenlc } \\
\text { (micro- } \\
\text { grams } \\
\text { per gram) }\end{array}$ & $\begin{array}{c}\text { Arsenic } \\
\text { - partlal } \\
\text { (micro- } \\
\text { grams } \\
\text { per gram) }\end{array}$ & $\begin{array}{c}\text { Barium } \\
\text { (micro- } \\
\text { grams } \\
\text { per gram) }\end{array}$ & $\begin{array}{l}\text { Beryllium } \\
\text { (micro- } \\
\text { grams } \\
\text { per gram) }\end{array}$ & $\begin{array}{c}\text { Bismuth } \\
\text { (micro- } \\
\text { grams } \\
\text { per gram) }\end{array}$ & $\begin{array}{l}\text { Bismuth } \\
\text { - partial } \\
\text { (micro- } \\
\text { grams } \\
\text { per gram) }\end{array}$ \\
\hline 87 & 1 & 5.6 & $<0.6$ & $<10$ & 2.0 & 470 & $<1$ & $<10$ & $<0.6$ \\
\hline 88 & 1 & 6.6 & $<.6$ & $<10$ & 3.9 & 560 & 2 & $<10$ & $<.6$ \\
\hline 89 & 1 & 5.5 & $<.6$ & $<10$ & 2.8 & 400 & $<1$ & $<10$ & $<.6$ \\
\hline 89 & 2 & 5.6 & $<.6$ & $<10$ & 1.8 & 420 & $<1$ & $<10$ & $<.6$ \\
\hline 89 & 2 & 5.6 & $<.6$ & $<10$ & 2.9 & 410 & $<1$ & $<10$ & $<.6$ \\
\hline 90 & 1 & 6.1 & $<.6$ & $<10$ & 2.9 & 460 & $<1$ & $<10$ & $<.6$ \\
\hline 91 & 1 & 5.5 & $<.6$ & $<10$ & 2.4 & 520 & 2 & $<10$ & $<.6$ \\
\hline 92 & 1 & 6.2 & $<.6$ & $<10$ & 2.8 & 530 & $<1$ & $<10$ & $<.6$ \\
\hline 93 & 1 & 7.5 & .7 & $<10$ & 3.7 & 630 & 2 & $<10$ & $<.6$ \\
\hline 94 & 1 & 5.7 & $<.6$ & $<10$ & 2.0 & 450 & 2 & $<10$ & $<.6$ \\
\hline 95 & 1 & 6.8 & $<.6$ & $<10$ & 3.7 & 590 & 2 & $<10$ & $<.6$ \\
\hline 96 & 1 & 5.6 & $<.6$ & $<10$ & 2.5 & 540 & 2 & $<10$ & $<.6$ \\
\hline 97 & 1 & 5.6 & $<.6$ & $<10$ & 2.4 & 480 & 2 & $<10$ & $<.6$ \\
\hline
\end{tabular}


Table 6. Inorganic-constituent concentrations in fine-fraction streambed sediment for tributarles to Lake Champlain--Continued

\begin{tabular}{|c|c|c|c|c|c|c|c|c|c|}
\hline $\begin{array}{l}\text { Slte } \\
\text { number } 1\end{array}$ & $\begin{array}{l}\text { Deelgn } \\
\text { remarks }\end{array}$ & $\begin{array}{l}\text { Cadmilum } \\
\text { (mloro- } \\
\text { grams } \\
\text { per gram) }\end{array}$ & $\begin{array}{c}\text { Cadmium } \\
\text {-partial } \\
\text { (mloro- } \\
\text { grams } \\
\text { per gram) }\end{array}$ & $\begin{array}{l}\text { Calclum } \\
\text { (weight } \\
\text { percent) }\end{array}$ & $\begin{array}{l}\text { Cerium } \\
\text { (mloro- } \\
\text { grams } \\
\text { por gram) }\end{array}$ & $\begin{array}{c}\text { Chromlum } \\
\text { (mioro- } \\
\text { grame } \\
\text { per gram) }\end{array}$ & $\begin{array}{l}\text { Cobalt } \\
\text { (mlcro- } \\
\text { grams } \\
\text { per gram) }\end{array}$ & $\begin{array}{l}\text { Copper } \\
\text { (mlero- } \\
\text { grams } \\
\text { per gram) }\end{array}$ & $\begin{array}{l}\text { Copper } \\
\text { - partial } \\
\text { (mloro- } \\
\text { grams } \\
\text { per gram) }\end{array}$ \\
\hline 1 & 1 & $<2$ & 0.39 & 1.4 & 71 & 71 & 17 & 20 & 22 \\
\hline 1 & 2 & $<2$ & .37 & 1.5 & 63 & 62 & 15 & 19 & 20 \\
\hline 1 & 2 & $<2$ & .35 & 1.5 & 69 & 63 & 15 & 19 & 20 \\
\hline 4 & 1 & $<2$ & .20 & 1.0 & 85 & 84 & 16 & 17 & 18 \\
\hline 4 & 2 & $<2$ & .20 & 1.1 & 87 & 79 & 14 & 14 & 16 \\
\hline 4 & 2 & $<2$ & .19 & 1.1 & 80 & 82 & 13 & 15 & 15 \\
\hline 5 & 1 & $<2$ & .14 & 2.0 & 90 & 73 & 11 & 9 & 11 \\
\hline 6 & 1 & 3 & 2.1 & 2.8 & 26 & 52 & 12 & 51 & 34 \\
\hline 8 & 3 & $<2$ & -- & 1.5 & 92 & 160 & 19 & 15 & -- \\
\hline 8 & 1 & $<2$ & .27 & 1.5 & 94 & 110 & 20 & 14 & 18 \\
\hline 9 & 2 & $<2$ & .31 & 1.5 & 59 & 50 & 11 & 14 & 14 \\
\hline 9 & 1 & $<2$ & .27 & 1.5 & 55 & 46 & 10 & 11 & 11 \\
\hline 9 & 2 & $<2$ & .29 & 1.5 & 62 & 51 & 11 & 14 & 14 \\
\hline 10 & 1 & $<2$ & .35 & 4.4 & 61 & 65 & 11 & 14 & 15 \\
\hline 11 & 2 & $<2$ & .21 & 1.3 & 92 & 99 & 13 & 12 & 13 \\
\hline 11 & 1 & $<2$ & .27 & 1.2 & 88 & 89 & 15 & 14 & 15 \\
\hline 11 & 2 & $<2$ & .22 & 1.3 & 90 & 93 & 13 & 11 & 13 \\
\hline 13 & 1 & $<2$ & .17 & 4.3 & 61 & 54 & 11 & 11 & 12 \\
\hline 14 & 1 & $<2$ & .37 & 1.6 & 77 & 75 & 16 & 28 & 30 \\
\hline 14 & 3 & $<2$ & -- & 1.6 & 75 & 70 & 13 & 23 & -- \\
\hline 15 & 3 & $<2$ & -- & 1.8 & 64 & 130 & 18 & 33 & -- \\
\hline 16 & 1 & $<2$ & .12 & 2.2 & 54 & 44 & 8 & 6 & 5.8 \\
\hline 18 & 1 & $<2$ & .27 & 2.5 & 82 & 84 & 15 & 18 & 20 \\
\hline 19 & 1 & $<2$ & .24 & 1.4 & 65 & 54 & 11 & 15 & 15 \\
\hline 20 & 1 & $<2$ & .13 & 3.0 & 75 & 60 & 10 & 9 & 10 \\
\hline 21 & 1 & $<2$ & .23 & 2.9 & 58 & 70 & 12 & 11 & 8.3 \\
\hline 26 & 1 & $<2$ & .44 & 2.4 & 64 & 100 & 10 & 29 & 28 \\
\hline 26 & 2 & $<2$ & .51 & 2.4 & 67 & 91 & 10 & 41 & 40 \\
\hline 26 & 2 & $<2$ & .55 & 2.3 & 68 & 92 & 12 & 42 & 42 \\
\hline 29 & 1 & $<2$ & .12 & 1.2 & 87 & 67 & 11 & 9 & 9.7 \\
\hline 30 & 1 & $<2$ & .69 & 1.4 & 110 & 64 & 13 & 18 & 18 \\
\hline 31 & 1 & $<2$ & .10 & 1.2 & 95 & 73 & 14 & 12 & 13 \\
\hline 32 & 1 & $<2$ & .08 & 2.1 & 71 & 62 & 6 & 5 & 4.0 \\
\hline 32 & 2 & $<2$ & -- & 2.3 & 79 & 59 & 7 & 6 & -- \\
\hline 32 & 2 & $<2$ & -- & 2.2 & 72 & 63 & 8 & 19 & -- \\
\hline 33 & 1 & $<2$ & .21 & 4.6 & 69 & 60 & 13 & 11 & 12 \\
\hline 34 & 1 & $<2$ & .35 & .81 & 83 & 190 & 22 & 17 & 20 \\
\hline 36 & 1 & $<2$ & .12 & 1.9 & 58 & 45 & 6 & 5 & 4.7 \\
\hline 37 & 1 & $<2$ & .14 & 1.2 & 100 & 73 & 13 & 10 & 12 \\
\hline 39 & 1 & $<2$ & .17 & 1.1 & 96 & 75 & 15 & 13 & 14 \\
\hline 41 & 1 & $<2$ & .11 & 1.1 & 90 & 67 & 12 & 11 & 12 \\
\hline 42 & 1 & $<2$ & .15 & .95 & 98 & 80 & 19 & 15 & 16 \\
\hline 43 & 1 & $<2$ & .40 & 2.5 & 53 & 140 & 14 & 11 & 11 \\
\hline 43 & 2 & $<2$ & -- & 2.9 & 62 & 250 & 17 & 16 & -- \\
\hline 43 & 2 & $<2$ & -- & 2.9 & 58 & 230 & 18 & 23 & -- \\
\hline
\end{tabular}


Table 6. Inorganic-constituent concentrations in fine-fraction streambed sediment for tributaries to Lake Champlain--Continued

\begin{tabular}{|c|c|c|c|c|c|c|c|c|c|}
\hline $\begin{array}{c}\text { Site } \\
\text { number }^{1}\end{array}$ & $\underset{\text { remarks }}{\text { Design }}$ & $\begin{array}{l}\text { Cadmlum } \\
\text { (micro- } \\
\text { grams } \\
\text { per gram) }\end{array}$ & $\begin{array}{l}\text { Cadmlum } \\
\text {-partial } \\
\text { (mlcro- } \\
\text { grams } \\
\text { por gram) }\end{array}$ & $\begin{array}{l}\text { Calclum } \\
\text { (welght } \\
\text { percent) }\end{array}$ & $\begin{array}{l}\text { Cerium } \\
\text { (mlcro- } \\
\text { grams } \\
\text { per gram) }\end{array}$ & $\begin{array}{c}\text { Chromium } \\
\text { (mlcro- } \\
\text { grams } \\
\text { per gram) }\end{array}$ & $\begin{array}{l}\text { Cobalt } \\
\text { (micro- } \\
\text { grams } \\
\text { por gram) }\end{array}$ & $\begin{array}{l}\text { Copper } \\
\text { (mlcro- } \\
\text { grame } \\
\text { per gram) }\end{array}$ & $\begin{array}{l}\text { Copper } \\
\text { - partial } \\
\text { (mlcro- } \\
\text { grams } \\
\text { per gram) }\end{array}$ \\
\hline 44 & 1 & $<2$ & 0.19 & 1.2 & 86 & 73 & 14 & 17 & 20 \\
\hline 46 & 1 & $<2$ & .29 & 1.4 & 77 & 140 & 17 & 25 & 26 \\
\hline 47 & 1 & $<2$ & .13 & 2.2 & 44 & 41 & 6 & 6 & 4.8 \\
\hline 48 & 1 & $<2$ & .33 & 1.6 & 83 & 74 & 14 & 15 & 17 \\
\hline 49 & 1 & $<2$ & .09 & 2.0 & 51 & 52 & 7 & 6 & 5.2 \\
\hline 51 & 1 & $<2$ & .21 & 1.5 & 81 & 65 & 12 & 12 & 13 \\
\hline 52 & 1 & $<2$ & .19 & 1.6 & 80 & 65 & 14 & 15 & 17 \\
\hline 53 & 2 & $<2$ & .18 & 3.5 & 60 & 65 & 16 & 9 & 9.8 \\
\hline 53 & 1 & $<2$ & .10 & 3.9 & 60 & 68 & 15 & 6 & 6.9 \\
\hline 53 & 2 & $<2$ & .18 & 3.5 & 63 & 63 & 17 & 6 & 11 \\
\hline 55 & 1 & $<2$ & .22 & 1.6 & 92 & 96 & 22 & 31 & 37 \\
\hline 56 & 1 & $<2$ & .18 & 1.2 & 100 & 78 & 18 & 18 & 21 \\
\hline 57 & 1 & $<2$ & .13 & 1.2 & 91 & 60 & 12 & 10 & 12 \\
\hline 59 & 1 & $<2$ & .22 & 3.6 & 71 & 110 & 16 & 17 & 20 \\
\hline 61 & 1 & $<2$ & .17 & 2.6 & 54 & 54 & 14 & 9 & 11 \\
\hline 62 & 1 & $<2$ & .39 & .96 & 80 & 59 & 14 & 16 & 19 \\
\hline 63 & 1 & $<2$ & .18 & 2.5 & 62 & 67 & 19 & 14 & 14 \\
\hline 64 & 3 & $<2$ & -- & 3.5 & 40 & 78 & 17 & 11 & -- \\
\hline 65 & 3 & $<2$ & -- & 2.7 & 53 & 53 & 17 & 13 & -- \\
\hline 66 & 1 & $<2$ & .30 & 3.0 & 60 & 84 & 18 & 16 & 17 \\
\hline 66 & 3 & $<2$ & -- & 3.3 & 53 & 58 & 16 & 13 & -- \\
\hline 67 & 1 & $<2$ & .39 & 3.3 & 510 & 81 & 28 & 27 & 32 \\
\hline 67 & 3 & $<2$ & - & 3.3 & 400 & 50 & 24 & 44 & -- \\
\hline 68 & 3 & $<2$ & -- & 3.3 & 470 & 45 & 23 & 120 & -- \\
\hline 69 & 1 & $<2$ & .23 & 3.5 & 110 & 77 & 16 & 13 & 14 \\
\hline 70 & 1 & $<2$ & .22 & 1.4 & 110 & 97 & 23 & 26 & 30 \\
\hline 71 & 1 & $<2$ & .23 & 1.6 & 110 & 94 & 23 & 27 & 32 \\
\hline 72 & 1 & $<2$ & .28 & 4.1 & 110 & 170 & 15 & 16 & 15 \\
\hline 73 & 1 & $<2$ & .14 & 4.2 & 70 & 40 & 10 & 8 & 8.6 \\
\hline 75 & 1 & $<2$ & .17 & 3.9 & 68 & 69 & 16 & 17 & 19 \\
\hline 76 & 1 & $<2$ & .16 & 4.7 & 70 & 63 & 16 & 17 & 19 \\
\hline 77 & 1 & $<2$ & .23 & 3.0 & 82 & 84 & 19 & 28 & 29 \\
\hline 78 & 1 & $<2$ & .47 & 2.5 & 83 & 170 & 15 & 48 & 47 \\
\hline 78 & 2 & $<2$ & .45 & 2.6 & 87 & 160 & 15 & 49 & 49 \\
\hline 78 & 2 & $<2$ & .45 & 2.6 & 88 & 150 & 15 & 48 & 49 \\
\hline 80 & 1 & $<2$ & .18 & 3.7 & 85 & 79 & 18 & 23 & 24 \\
\hline 81 & 1 & $<2$ & .38 & 1.0 & 79 & 89 & 23 & 23 & 24 \\
\hline 82 & 1 & $<2$ & .43 & 1.4 & 110 & 98 & 27 & 30 & 33 \\
\hline 83 & 1 & $<2$ & .25 & 2.4 & 77 & 72 & 16 & 14 & 16 \\
\hline 84 & 1 & $<2$ & .19 & 3.6 & 69 & 66 & 15 & 20 & 20 \\
\hline 85 & 3 & $<2$ & -- & 6.3 & 52 & 51 & 12 & 18 & -- \\
\hline 85 & 1 & $<2$ & .18 & 7.9 & 51 & 52 & 8 & 13 & 13 \\
\hline 86 & 1 & $<2$ & .32 & 1.6 & 92 & 91 & 21 & 25 & 27 \\
\hline 87 & 1 & $<2$ & .39 & 4.6 & 67 & 74 & 10 & 16 & 16 \\
\hline
\end{tabular}


Table 6. Inorganic-constituent concentrations in fine-fraction streambed sediment for tributaries to Lake Champlain--ContInued

\begin{tabular}{|c|c|c|c|c|c|c|c|c|c|}
\hline $\begin{array}{l}\text { Site } \\
\text { number } 1\end{array}$ & $\begin{array}{l}\text { Design } \\
\text { remarks }\end{array}$ & $\begin{array}{l}\text { Cadmlum } \\
\text { (micro- } \\
\text { grams } \\
\text { per gram) }\end{array}$ & $\begin{array}{l}\text { Cadmium } \\
\text {-partial } \\
\text { (micro- } \\
\text { grams } \\
\text { per gram) }\end{array}$ & $\begin{array}{l}\text { Calclum } \\
\text { (weight } \\
\text { percent) }\end{array}$ & $\begin{array}{l}\text { Cerium } \\
\text { (micro- } \\
\text { grams } \\
\text { per gram) }\end{array}$ & $\begin{array}{c}\text { Chromlum } \\
\text { (micro- } \\
\text { grams } \\
\text { per gram) }\end{array}$ & $\begin{array}{l}\text { Cobalt } \\
\text { (mlcro- } \\
\text { grams } \\
\text { per gram) }\end{array}$ & $\begin{array}{l}\text { Copper } \\
\text { (mlcro- } \\
\text { grams } \\
\text { per gram) }\end{array}$ & $\begin{array}{l}\text { Copper } \\
\text { - partial } \\
\text { (micro- } \\
\text { grams } \\
\text { per gram) }\end{array}$ \\
\hline 88 & 1 & $<2$ & 0.15 & 1.7 & 93 & 78 & 19 & 21 & 21 \\
\hline 89 & 1 & $<2$ & .11 & 2.0 & 190 & 59 & 13 & 13 & 13 \\
\hline 89 & 2 & $<2$ & .08 & 2.0 & 110 & 55 & 14 & 14 & 10 \\
\hline 89 & 2 & $<2$ & .12 & 2.0 & 120 & 54 & 14 & 14 & 15 \\
\hline 90 & 1 & $<2$ & .17 & 1.9 & 84 & 59 & 15 & 17 & 18 \\
\hline 91 & 1 & $<2$ & 1.3 & 2.3 & 76 & 81 & 17 & 18 & 19 \\
\hline 92 & 1 & $<2$ & 1.0 & 2.1 & 67 & 110 & 24 & 27 & 27 \\
\hline 93 & 1 & $<2$ & .26 & .84 & 100 & 81 & 22 & 25 & 26 \\
\hline 94 & 1 & $<2$ & 2.4 & 5.5 & 58 & 180 & 44 & 24 & 19 \\
\hline 95 & 1 & $<2$ & .28 & .60 & 74 & 63 & 15 & 25 & 26 \\
\hline 96 & 1 & $<2$ & 1.6 & 3.2 & 60 & 77 & 24 & 19 & 20 \\
\hline 97 & 1 & $<2$ & .81 & 2.9 & 94 & 93 & 18 & 23 & 24 \\
\hline
\end{tabular}




\begin{tabular}{|c|c|c|c|c|c|c|c|c|c|}
\hline $\begin{array}{c}\text { Slte } \\
\text { number }\end{array}$ & $\underset{\text { remarke }}{\text { Deelgn }}$ & $\begin{array}{c}\text { Europlum } \\
\text { (micro- } \\
\text { grams } \\
\text { per gram) }\end{array}$ & $\begin{array}{l}\text { Gallium } \\
\text { (micro- } \\
\text { grams } \\
\text { por gram) }\end{array}$ & $\begin{array}{l}\text { Gold } \\
\text { (mlcro- } \\
\text { grams } \\
\text { per gram) }\end{array}$ & $\begin{array}{l}\text { Gold } \\
\text {-partial } \\
\text { (micro- } \\
\text { grams } \\
\text { per gram) }\end{array}$ & $\begin{array}{l}\text { Holmlum } \\
\text { (mlcro- } \\
\text { grams } \\
\text { per gram) }\end{array}$ & $\begin{array}{l}\text { Iron } \\
\text { (wolght } \\
\text { porcent) }\end{array}$ & $\begin{array}{l}\text { Lanthanum } \\
\text { (mlcro- } \\
\text { grams } \\
\text { per gram) }\end{array}$ & $\begin{array}{l}\text { Lead } \\
\text { (micro- } \\
\text { grams } \\
\text { per gram) }\end{array}$ \\
\hline 1 & 1 & $<2$ & 16 & $<8$ & $<0.1$ & $<4$ & 3.6 & 35 & 19 \\
\hline 1 & 2 & $<2$ & 15 & $<8$ & $<.1$ & $<4$ & 3.3 & 31 & 18 \\
\hline 1 & 2 & $<2$ & 15 & $<8$ & $<.1$ & $<4$ & 3.3 & 32 & 20 \\
\hline 4 & 1 & $<2$ & 18 & $<8$ & $<.1$ & $<4$ & 3.9 & 40 & 14 \\
\hline 4 & 2 & $<2$ & 17 & $<8$ & $<.1$ & $<4$ & 3.6 & 41 & 15 \\
\hline 4 & 2 & $<2$ & 19 & $<8$ & $<.1$ & $<4$ & 3.5 & 37 & 16 \\
\hline 5 & 1 & $<2$ & 13 & $<8$ & $<.1$ & $<4$ & 3.9 & 43 & 11 \\
\hline 6 & 1 & $<2$ & 8 & $<8$ & $<.1$ & $<4$ & 2.9 & 13 & 230 \\
\hline 8 & 3 & $<2$ & 19 & $<8$ & -- & $<4$ & 5.5 & 49 & 320 \\
\hline 8 & 1 & $<2$ & 23 & $<8$ & $<.1$ & $<4$ & 5.5 & 44 & 280 \\
\hline 9 & 2 & $<2$ & 13 & $<8$ & $<.1$ & $<4$ & 2.5 & 28 & 22 \\
\hline 9 & 1 & $<2$ & 13 & $<8$ & $<.1$ & $<4$ & 2.3 & 25 & 21 \\
\hline 9 & 2 & $<2$ & 14 & $<8$ & $<.1$ & $<4$ & 2.5 & 29 & 23 \\
\hline 10 & 1 & $<2$ & 13 & $<8$ & $<.1$ & $<4$ & 3.0 & 28 & 41 \\
\hline 11 & 2 & $<2$ & 16 & $<8$ & $<.1$ & $<4$ & 4.1 & 45 & 16 \\
\hline 11 & 1 & $<2$ & 16 & $<8$ & $<.1$ & $<4$ & 4.2 & 45 & 14 \\
\hline 11 & 2 & $<2$ & 15 & $<8$ & $<.1$ & $<4$ & 4.1 & 44 & 12 \\
\hline 13 & 1 & $<2$ & 13 & $<8$ & $<.1$ & $<4$ & 2.6 & 28 & 21 \\
\hline 14 & 1 & $<2$ & 17 & $<8$ & $<.1$ & $<4$ & 3.7 & 36 & 46 \\
\hline 14 & 3 & $<2$ & 16 & $<8$ & -- & $<4$ & 3.6 & 40 & 42 \\
\hline 15 & 3 & $<2$ & 19 & $<8$ & -- & $<4$ & 4.2 & 35 & 53 \\
\hline 16 & 1 & $<2$ & 12 & $<8$ & $<.1$ & $<4$ & 2.1 & 25 & 16 \\
\hline 18 & 1 & $<2$ & 18 & $<8$ & $<.1$ & $<4$ & 3.7 & 38 & 13 \\
\hline 19 & 1 & $<2$ & 15 & $<8$ & $<.1$ & $<4$ & 2.8 & 30 & 16 \\
\hline 20 & 1 & $<2$ & 13 & $<8$ & $<.1$ & $<4$ & 2.9 & 34 & 16 \\
\hline 21 & 1 & $<2$ & 18 & $<8$ & $<.1$ & $<4$ & 3.3 & 26 & 22 \\
\hline 26 & 1 & $<2$ & 12 & $<8$ & $<.1$ & $<4$ & 3.8 & 29 & 100 \\
\hline 26 & 2 & $<2$ & 13 & $<8$ & $<.1$ & $<4$ & 4.1 & 31 & 130 \\
\hline 26 & 2 & $<2$ & 13 & $<8$ & $<.1$ & $<4$ & 4.1 & 31 & 100 \\
\hline 29 & 1 & $<2$ & 14 & $<8$ & $<.1$ & $<4$ & 3.4 & 42 & 10 \\
\hline 30 & 1 & $<2$ & 16 & $<8$ & $<.1$ & $<4$ & 4.7 & 41 & 36 \\
\hline 31 & 1 & $<2$ & 16 & $<8$ & $<.1$ & $<4$ & 4.0 & 46 & 9 \\
\hline 32 & 1 & $<2$ & 11 & $<8$ & $<.1$ & $<4$ & 3.1 & 31 & 12 \\
\hline 32 & 2 & $<2$ & 12 & $<8$ & -- & $<4$ & 3.3 & 33 & 11 \\
\hline 32 & 2 & $<2$ & 11 & $<8$ & -- & $<4$ & 3.3 & 31 & 11 \\
\hline 33 & 1 & $<2$ & 16 & $<8$ & $<.1$ & $<4$ & 3.0 & 31 & 13 \\
\hline 34 & 1 & $<2$ & 22 & $<8$ & $<.1$ & $<4$ & 4.6 & 42 & 25 \\
\hline 36 & 1 & $<2$ & 12 & $<8$ & $<.1$ & $<4$ & 3.1 & 26 & 12 \\
\hline 37 & 1 & $<2$ & 16 & $<8$ & $<.1$ & $<4$ & 4.0 & 49 & 11 \\
\hline 39 & 1 & $<2$ & 17 & $<8$ & $<.1$ & $<4$ & 3.8 & 46 & 13 \\
\hline 41 & 1 & $<2$ & 15 & $<8$ & $<.1$ & $<4$ & 3.7 & 43 & 14 \\
\hline 42 & 1 & $<2$ & 20 & $<8$ & $<.1$ & $<4$ & 4.6 & 46 & 16 \\
\hline 43 & 1 & $<2$ & 15 & $<8$ & $<.1$ & $<4$ & 4.5 & 23 & 19 \\
\hline 43 & 2 & $<2$ & 16 & $<8$ & -- & $<4$ & 7.1 & 28 & 19 \\
\hline 43 & 2 & $<2$ & 15 & $<8$ & -- & $<4$ & 7.7 & 27 & 18 \\
\hline
\end{tabular}


Table 6. Inorganic-constituent concentrations in fine-fraction streambed sediment for tributaries to Lake Champlain--Continued

\begin{tabular}{|c|c|c|c|c|c|c|c|c|c|}
\hline $\begin{array}{c}\text { Slte } \\
\text { number }{ }^{1}\end{array}$ & $\underset{\text { remarks }}{\text { Design }}$ & $\begin{array}{c}\text { Europlum } \\
\text { (mlcro- } \\
\text { grams } \\
\text { per gram) }\end{array}$ & $\begin{array}{l}\text { Galllum } \\
\text { (micro- } \\
\text { grams } \\
\text { per gram) }\end{array}$ & $\begin{array}{l}\text { Gold } \\
\text { (micro- } \\
\text { grams } \\
\text { per gram) }\end{array}$ & $\begin{array}{c}\text { Goid } \\
\text {-partiai } \\
\text { (micro- } \\
\text { grams } \\
\text { per gram) }\end{array}$ & $\begin{array}{l}\text { Hoimlum } \\
\text { (micro- } \\
\text { grams } \\
\text { per gram) }\end{array}$ & $\begin{array}{l}\text { iron } \\
\text { (welght } \\
\text { percent) }\end{array}$ & $\begin{array}{l}\text { Lanthanum } \\
\text { (micro- } \\
\text { grams } \\
\text { per gram) }\end{array}$ & $\begin{array}{l}\text { Lead } \\
\text { (mlcro- } \\
\text { grams } \\
\text { per gram) }\end{array}$ \\
\hline 44 & 1 & $<2$ & 16 & $<8$ & $<0.1$ & $<4$ & 3.7 & 43 & 17 \\
\hline 46 & 1 & $<2$ & 17 & $<8$ & $<.1$ & $<4$ & 4.4 & 40 & 23 \\
\hline 47 & 1 & $<2$ & 12 & $<8$ & $<.1$ & $<4$ & 2.1 & 20 & 14 \\
\hline 48 & 1 & $<2$ & 16 & $<8$ & $<.1$ & $<4$ & 3.7 & 38 & 21 \\
\hline 49 & 1 & $<2$ & 12 & $<8$ & $<.1$ & $<4$ & 2.6 & 23 & 15 \\
\hline 51 & 1 & $<2$ & 15 & $<8$ & $<.1$ & $<4$ & 3.4 & 36 & 17 \\
\hline 52 & 1 & $<2$ & 16 & $<8$ & $<.1$ & $<4$ & 3.6 & 37 & 12 \\
\hline 53 & 2 & $<2$ & 17 & $<8$ & $<.1$ & $<4$ & 5.4 & 29 & 11 \\
\hline 53 & 1 & $<2$ & 17 & $<8$ & $<.1$ & $<4$ & 5.8 & 29 & 9 \\
\hline 53 & 2 & $<2$ & 17 & $<8$ & $<.1$ & $<4$ & 5.4 & 31 & 11 \\
\hline 55 & 1 & $<2$ & 21 & $<8$ & $<.1$ & $<4$ & 4.5 & 44 & 16 \\
\hline 56 & 1 & $<2$ & 19 & $<8$ & $<.1$ & $<4$ & 3.8 & 45 & 14 \\
\hline 57 & 1 & $<2$ & 14 & $<8$ & $<.1$ & $<4$ & 3.7 & 44 & 11 \\
\hline 59 & 1 & $<2$ & 17 & $<8$ & $<.1$ & $<4$ & 3.6 & 34 & 12 \\
\hline 61 & 1 & $<2$ & 16 & $<8$ & $<.1$ & $<4$ & 4.3 & 26 & 16 \\
\hline 62 & 1 & $<2$ & 17 & $<8$ & $<.1$ & $<4$ & 3.6 & 41 & 17 \\
\hline 63 & 1 & $<2$ & 17 & $<8$ & $<.1$ & $<4$ & 4.4 & 29 & 14 \\
\hline 64 & 3 & $<2$ & 16 & $<8$ & -- & $<4$ & 4.3 & 25 & 13 \\
\hline 65 & 3 & $<2$ & 16 & $<8$ & -- & $<4$ & 4.2 & 34 & 14 \\
\hline 66 & 1 & $<2$ & 16 & $<8$ & $<.1$ & $<4$ & 4.3 & 32 & 11 \\
\hline 66 & 3 & $<2$ & 15 & $<8$ & -- & $<4$ & 4.5 & 32 & 12 \\
\hline 67 & 1 & 3 & 19 & $<8$ & $<.1$ & 5 & 9.4 & 290 & 19 \\
\hline 67 & 3 & 3 & 17 & $<8$ & -- & $<4$ & 8.2 & 250 & 25 \\
\hline 68 & 3 & 3 & 18 & $<8$ & -- & 5 & 8.5 & 300 & 13 \\
\hline 69 & 1 & $<2$ & 15 & $<8$ & $<.1$ & $<4$ & 4.8 & 52 & 21 \\
\hline 70 & 1 & $<2$ & 21 & $<8$ & $<.1$ & $<4$ & 4.2 & 50 & 15 \\
\hline 71 & 1 & $<2$ & 20 & $<8$ & $<.1$ & $<4$ & 4.4 & 46 & 18 \\
\hline 72 & 1 & $<2$ & 15 & $<8$ & $<.1$ & $<4$ & 4.3 & 55 & 15 \\
\hline 73 & 1 & $<2$ & 14 & $<8$ & $<.1$ & $<4$ & 4.5 & 34 & 10 \\
\hline 75 & 1 & $<2$ & 16 & $<8$ & $<.1$ & $<4$ & 3.6 & 31 & 15 \\
\hline 76 & 1 & $<2$ & 15 & $<8$ & $<.1$ & $<4$ & 3.5 & 33 & 14 \\
\hline 77 & 1 & $<2$ & 18 & $<8$ & $<.1$ & $<4$ & 3.8 & 37 & 15 \\
\hline 78 & 1 & $<2$ & 14 & $<8$ & $<.1$ & $<4$ & 4.0 & 43 & 77 \\
\hline 78 & 2 & $<2$ & 14 & $<8$ & $<.1$ & $<4$ & 4.1 & 44 & 79 \\
\hline 78 & 2 & $<2$ & 15 & $<8$ & $<.1$ & $<4$ & 4.2 & 44 & 79 \\
\hline 80 & 1 & $<2$ & 16 & $<8$ & $<.1$ & $<4$ & 3.6 & 37 & 15 \\
\hline 81 & 1 & $<2$ & 29 & $<8$ & $<.1$ & $<4$ & 4.6 & 39 & 17 \\
\hline 82 & 1 & $<2$ & 22 & $<8$ & $<.1$ & $<4$ & 4.7 & 49 & 18 \\
\hline 83 & 1 & $<2$ & 16 & $<8$ & $<.1$ & $<4$ & 3.3 & 35 & 14 \\
\hline 84 & 1 & $<2$ & 17 & $<8$ & $<.1$ & $<4$ & 3.4 & 32 & 16 \\
\hline 85 & 3 & $<2$ & 14 & $<8$ & -- & $<4$ & 4.4 & 31 & 14 \\
\hline 85 & 1 & $<2$ & 12 & $<8$ & $<.1$ & $<4$ & 2.4 & 26 & 10 \\
\hline 86 & 1 & $<2$ & 20 & $<8$ & $<.1$ & $<4$ & 4.6 & 41 & 21 \\
\hline 87 & 1 & $<2$ & 14 & $<8$ & $<.1$ & $<4$ & 3.1 & 37 & 25 \\
\hline
\end{tabular}


Table 6. Inorganic-constituent concentrations in fine-fraction streambed sediment for tributaries to Lake Champlain--Continued

\begin{tabular}{|c|c|c|c|c|c|c|c|c|c|}
\hline $\begin{array}{l}\text { Site } \\
\text { number }\end{array}$ & $\begin{array}{l}\text { Design } \\
\text { remarke }\end{array}$ & $\begin{array}{c}\text { Europium } \\
\text { (micro- } \\
\text { grams } \\
\text { per gram) }\end{array}$ & $\begin{array}{l}\text { Gaiiium } \\
\text { (micro- } \\
\text { grams } \\
\text { per gram) }\end{array}$ & $\begin{array}{c}\text { Goid } \\
\text { (micro- } \\
\text { grams } \\
\text { per gram) }\end{array}$ & $\begin{array}{l}\text { Goid } \\
\text {-partial } \\
\text { (micro- } \\
\text { grams } \\
\text { per gram) }\end{array}$ & $\begin{array}{l}\text { Hoimium } \\
\text { (micro- } \\
\text { grams } \\
\text { per gram) }\end{array}$ & $\begin{array}{l}\text { iron } \\
\text { (weight } \\
\text { percent) }\end{array}$ & $\begin{array}{l}\text { Lanthanum } \\
\text { (micro- } \\
\text { grame } \\
\text { per gram) }\end{array}$ & $\begin{array}{l}\text { Load } \\
\text { (mlcro- } \\
\text { grams } \\
\text { per gram) }\end{array}$ \\
\hline 88 & 1 & $<2$ & 17 & $<8$ & $<0.1$ & $<4$ & 3.8 & 41 & 18 \\
\hline 89 & 1 & $<2$ & 15 & $<8$ & $<.1$ & $<4$ & 3.7 & 86 & 12 \\
\hline 89 & 2 & $<2$ & 15 & $<8$ & $<.1$ & $<4$ & 3.5 & 52 & 13 \\
\hline 89 & 2 & $<2$ & 15 & $<8$ & $<.1$ & $<4$ & 3.5 & 55 & 12 \\
\hline 90 & 1 & $<2$ & 17 & $<8$ & $<.1$ & $<4$ & 4.0 & 39 & 15 \\
\hline 91 & 1 & $<2$ & 15 & $<8$ & $<.1$ & $<4$ & 3.7 & 41 & 32 \\
\hline 92 & 1 & $<2$ & 16 & $<8$ & $<.1$ & $<4$ & 4.3 & 32 & 37 \\
\hline 93 & 1 & $<2$ & 19 & $<8$ & $<.1$ & $<4$ & 4.4 & 42 & 24 \\
\hline 94 & 1 & $<2$ & 16 & $<8$ & $<.1$ & $<4$ & 3.6 & 26 & 33 \\
\hline 95 & 1 & $<2$ & 16 & $<8$ & $<.1$ & $<4$ & 3.7 & 38 & 21 \\
\hline 96 & 1 & $<2$ & 13 & $<8$ & $<.1$ & $<4$ & 2.9 & 29 & 25 \\
\hline 97 & 1 & $<2$ & 15 & $<8$ & $<.1$ & $<4$ & 3.6 & 47 & 28 \\
\hline
\end{tabular}


Table 6. Inorganic-constituent concentrations in fine-fraction streambed sediment for tributaries to Lake Champlain--Continued

\begin{tabular}{|c|c|c|c|c|c|c|c|c|c|}
\hline $\begin{array}{c}\text { Slte } \\
\text { number }^{1}\end{array}$ & $\begin{array}{c}\text { Deeign } \\
\text { remarks }\end{array}$ & $\begin{array}{l}\text { Lead } \\
\text { - partial } \\
\text { (micro- } \\
\text { grams } \\
\text { per gram) }\end{array}$ & $\begin{array}{l}\text { Lithlum } \\
\text { (micro- } \\
\text { grams } \\
\text { per gram) }\end{array}$ & $\begin{array}{c}\text { Magneslum } \\
\text { (welght } \\
\text { percent) }\end{array}$ & $\begin{array}{l}\text { Manganese } \\
\text { (mlero- } \\
\text { grame } \\
\text { per gram) }\end{array}$ & $\begin{array}{l}\text { Mercury } \\
\text { (micro- } \\
\text { grams } \\
\text { per gram) }\end{array}$ & $\begin{array}{c}\text { Molybdenum } \\
\text { (micro- } \\
\text { grams } \\
\text { per gram) }\end{array}$ & $\begin{array}{c}\text { Molybdenum } \\
\text {-partial } \\
\text { (micro- } \\
\text { grams } \\
\text { per gram) }\end{array}$ & $\begin{array}{c}\text { Neodymium } \\
\text { (mlcro- } \\
\text { grams } \\
\text { per gram) }\end{array}$ \\
\hline 1 & 1 & 17 & 34 & 1.1 & 610 & 0.16 & $<2$ & 0.75 & 34 \\
\hline 1 & 2 & 16 & 30 & .96 & 560 & .09 & $<2$ & .68 & 31 \\
\hline 1 & 2 & 15 & 30 & .98 & 570 & .10 & $<2$ & .66 & 32 \\
\hline 4 & 1 & 14 & 39 & 1.2 & 680 & .08 & $<2$ & .47 & 36 \\
\hline 4 & 2 & 13 & 36 & 1.1 & 560 & .18 & $<2$ & .49 & 37 \\
\hline 4 & 2 & 13 & 36 & 1.1 & 560 & .19 & $<2$ & .49 & 35 \\
\hline 5 & 1 & 8.9 & 21 & .81 & 870 & .32 & $<2$ & .59 & 41 \\
\hline 6 & 1 & 210 & 20 & .53 & 340 & .48 & 4 & 2.0 & 12 \\
\hline 8 & 3 & -- & 25 & .93 & 4,200 & .30 & $<2$ & -- & 47 \\
\hline 8 & 1 & 340 & 26 & .95 & 6,800 & .72 & $<2$ & 2.4 & 43 \\
\hline 9 & 2 & 18 & 18 & .83 & 510 & .19 & $<2$ & .59 & 29 \\
\hline 9 & 1 & 15 & 15 & .77 & 460 & .17 & $<2$ & .61 & 26 \\
\hline 9 & 2 & 18 & 18 & .83 & 510 & .16 & $<2$ & .59 & 30 \\
\hline 10 & 1 & 43 & 19 & 1.9 & 1,200 & .13 & $<2$ & 1.3 & 31 \\
\hline 11 & 2 & 12 & 28 & .95 & 1,100 & .45 & $<2$ & .44 & 43 \\
\hline 11 & 1 & 13 & 29 & .98 & 930 & .22 & $<2$ & 1.0 & 42 \\
\hline 11 & 2 & 12 & 27 & .93 & 1,100 & .37 & $<2$ & 1.6 & 43 \\
\hline 13 & 1 & 18 & 15 & 1.2 & 1,600 & .16 & $<2$ & .91 & 30 \\
\hline 14 & 1 & 53 & 29 & .93 & 1,200 & .76 & $<2$ & 1.1 & 37 \\
\hline 14 & 3 & -- & 27 & .91 & 860 & .79 & $<2$ & -- & 41 \\
\hline 15 & 3 & -- & 35 & 1.2 & 2,200 & .53 & $<2$ & -- & 33 \\
\hline 16 & 1 & 8.6 & 14 & .64 & 520 & .06 & $<2$ & .92 & 26 \\
\hline 18 & 1 & 13 & 27 & 1.1 & 2,400 & .28 & $<2$ & 1.7 & 38 \\
\hline 19 & 1 & 10 & 21 & .81 & 310 & .06 & $<2$ & 1.2 & 30 \\
\hline 20 & 1 & 9.6 & 14 & 1.0 & 800 & .20 & $<2$ & 1.3 & 35 \\
\hline 21 & 1 & 13 & 15 & 1.2 & 4,900 & .14 & $<2$ & 1.6 & 27 \\
\hline 25 & 1 & 98 & 15 & 1.2 & 1,500 & .43 & $<2$ & 2.3 & 28 \\
\hline 25 & 2 & 110 & 17 & 1.2 & 1,800 & .33 & $<2$ & 2.1 & 32 \\
\hline 25 & 2 & 120 & 16 & 1.2 & 1,800 & .25 & $<2$ & 2.2 & 31 \\
\hline 29 & 1 & 7.9 & 23 & .74 & 1,700 & .17 & $<2$ & .50 & 40 \\
\hline 30 & 1 & 32 & 18 & .65 & 3,300 & .06 & $<2$ & 1.6 & 43 \\
\hline 31 & 1 & 7.2 & 31 & 1.0 & 1,200 & .38 & $<2$ & .25 & 42 \\
\hline 32 & 1 & 4.9 & 9 & .93 & 1,000 & .07 & $<2$ & 1.5 & 33 \\
\hline 32 & 2 & -- & 8 & 1.0 & 1,100 & .19 & $<2$ & -- & 37 \\
\hline 32 & 2 & -- & 8 & .97 & 1,100 & .20 & $<2$ & -- & 35 \\
\hline 33 & 1 & 11 & 20 & 1.4 & 2,200 & .07 & $<2$ & 1.1 & 32 \\
\hline 34 & 1 & 28 & 42 & 1.2 & 3,000 & .54 & 3 & 6.9 & 38 \\
\hline 36 & 1 & 8.3 & 9 & .83 & 990 & .10 & $<2$ & .85 & 29 \\
\hline 37 & 1 & 8.4 & 29 & .89 & 950 & .21 & $<2$ & .52 & 46 \\
\hline 39 & 1 & 11 & 31 & .99 & 1,400 & .16 & $<2$ & .85 & 43 \\
\hline 41 & 1 & 10 & 27 & .78 & 1,400 & .08 & $<2$ & .50 & 41 \\
\hline 42 & 1 & 15 & 39 & 1.1 & 2,200 & .36 & $<2$ & .60 & 42 \\
\hline 43 & 1 & 18 & 11 & .95 & 1,500 & .29 & $<2$ & 5.0 & 28 \\
\hline 43 & 2 & -- & 12 & 1.2 & 1,400 & .33 & 8 & -- & 34 \\
\hline
\end{tabular}


Table 6. Inorganlc-constltuent concentrations In fine-fractlon streambed sediment for tributarles to Lake Champlain--Continued

\begin{tabular}{|c|c|c|c|c|c|c|c|c|c|}
\hline $\begin{array}{c}\text { Site } \\
\text { number }\end{array}$ & $\underset{\text { remarks }}{\text { Design }}$ & $\begin{array}{c}\text { Lead } \\
\text { - partial } \\
\text { (mlcro- } \\
\text { grams } \\
\text { per gram) }\end{array}$ & $\begin{array}{l}\text { Lithlum } \\
\text { (micro- } \\
\text { grams } \\
\text { per gram) }\end{array}$ & $\begin{array}{l}\text { Magnesium } \\
\text { (welght } \\
\text { percent) }\end{array}$ & $\begin{array}{l}\text { Manganese } \\
\text { (micro- } \\
\text { grams } \\
\text { per gram) }\end{array}$ & $\begin{array}{c}\text { Mercury } \\
\text { (mlcro- } \\
\text { grams } \\
\text { per gram) }\end{array}$ & $\begin{array}{c}\text { Molybdenum } \\
\text { (micro- } \\
\text { grams } \\
\text { per gram) }\end{array}$ & $\begin{array}{c}\text { lolybdenum } \\
\text {-partial } \\
\text { (micro- } \\
\text { grams } \\
\text { per gram) }\end{array}$ & $\begin{array}{c}\text { Neodymium } \\
\text { (micro- } \\
\text { grams } \\
\text { per gram) }\end{array}$ \\
\hline 43 & 2 & -- & 12 & 1.2 & 1,400 & 0.28 & 7 & -- & 34 \\
\hline 44 & 1 & 14 & 32 & .83 & 940 & .17 & $<2$ & .60 & 40 \\
\hline 46 & 1 & 23 & 39 & 1.1 & 1,300 & .43 & $<2$ & 5.1 & 37 \\
\hline 47 & 1 & 8.1 & 10 & .97 & 710 & .08 & $<2$ & .06 & 21 \\
\hline 48 & 1 & 22 & 26 & .84 & 1,900 & .16 & $<2$ & .96 & 35 \\
\hline 49 & 1 & 8.5 & 10 & .76 & 700 & .03 & $<2$ & .13 & 25 \\
\hline 51 & 1 & 15 & 23 & .82 & 1,200 & .20 & $<2$ & 1.2 & 35 \\
\hline 52 & 1 & 13 & 27 & 1.0 & 840 & .25 & $<2$ & .81 & 36 \\
\hline 53 & 2 & 13 & 15 & 1.4 & 1,200 & .65 & $<2$ & .15 & 35 \\
\hline 53 & 1 & 8.5 & 11 & 1.5 & 1,200 & .05 & $<2$ & .59 & 37 \\
\hline 53 & 2 & 14 & 14 & 1.4 & 1,200 & .57 & $<2$ & 1.4 & 37 \\
\hline 55 & 1 & 18 & 45 & 1.5 & 1,000 & .20 & $<2$ & .69 & 39 \\
\hline 56 & 1 & 15 & 37 & 1.1 & 1,000 & .12 & $<2$ & .95 & 43 \\
\hline 57 & 1 & 11 & 31 & .89 & 750 & .21 & $<2$ & .97 & 43 \\
\hline 59 & 1 & 14 & 32 & 1.4 & 1,400 & .43 & $<2$ & 2.6 & 33 \\
\hline 61 & 1 & 17 & 18 & 1.0 & 1,400 & .20 & $<2$ & .72 & 29 \\
\hline 62 & 1. & 19 & 33 & .97 & 1,100 & .27 & $<2$ & .71 & 40 \\
\hline 63 & 1 & 13 & 23 & 1.2 & 1,000 & .26 & $<2$ & 1.0 & 32 \\
\hline 64 & 3 & -- & 12 & 1.1 & 1,200 & .35 & $<2$ & -- & 30 \\
\hline 65 & 3 & -- & 17 & .98 & 1,000 & .23 & $<2$ & -- & 38 \\
\hline 66 & 1 & 16 & 22 & 1.3 & 1,000 & 3.7 & $<2$ & 1.6 & 35 \\
\hline 66 & 3 & -- & 17 & 1.3 & 1,200 & .27 & $<2$ & -- & 34 \\
\hline 67 & 1 & 27 & 19 & 1.3 & 2,400 & .76 & $<2$ & 2.9 & 190 \\
\hline 67 & 3 & -- & 17 & 1.2 & 1,900 & .34 & $<2$ & -- & 170 \\
\hline 68 & 3 & -- & 17 & 1.2 & 1,700 & .37 & $<2$ & -- & 200 \\
\hline 69 & 1 & 23 & 15 & 1.1 & 1,200 & .32 & $<2$ & 1.7 & 49 \\
\hline 70 & 1 & 18 & 49 & 1.5 & 660 & .08 & $<2$ & .63 & 46 \\
\hline 71 & 1 & 20 & 45 & 1.5 & 760 & .09 & $<2$ & .77 & 40 \\
\hline 72 & 1 & 17 & 18 & 1.8 & 1,600 & .36 & 3 & 5.6 & 51 \\
\hline 73 & 1 & 9.2 & 14 & 1.3 & 1,200 & .35 & $<2$ & .60 & 37 \\
\hline 75 & 1 & 15 & 30 & 1.4 & 1,300 & .13 & $<2$ & 1.0 & 32 \\
\hline 76 & 1 & 13 & 30 & 1.4 & 720 & .09 & $<2$ & .64 & 33 \\
\hline 77 & 1 & 17 & 46 & 1.4 & 730 & .10 & $<2$ & .67 & 34 \\
\hline 78 & 1 & 84 & 29 & 1.3 & 540 & .38 & $<2$ & 1.4 & 39 \\
\hline 78 & 2 & 86 & 29 & 1.4 & 570 & .43 & $<2$ & 1.5 & 40 \\
\hline 78 & 2 & 85 & 28 & 1.4 & 560 & .45 & $<2$ & 1.5 & 41 \\
\hline 80 & 1 & 16 & 38 & 1.4 & 590 & .12 & $<2$ & .82 & 37 \\
\hline 81 & 1 & 21 & 50 & 1.2 & 7,000 & .10 & $<2$ & .74 & 37 \\
\hline 82 & 1 & 22 & 46 & 1.5 & 1,500 & .14 & $<2$ & 1.9 & 45 \\
\hline 83 & 1 & 16 & 27 & 1.3 & 1,600 & .36 & $<2$ & 1.7 & 35 \\
\hline 84 & 1 & 17 & 34 & 1.4 & 1,500 & .11 & $<2$ & .82 & 31 \\
\hline 85 & 3 & -- & 23 & 1.5 & 2,000 & .19 & $<2$ & -- & 31 \\
\hline 85 & 1 & 11 & 21 & 1.5 & 720 & .75 & $<2$ & .06 & 25 \\
\hline 86 & 1 & 22 & 45 & 1.3 & 1,500 & .25 & $<2$ & 1.2 & 36 \\
\hline
\end{tabular}


Table 6. Inorganic-constituent concentrations in fine-fraction streambed sediment for tributaries to Lake Champlain--Continued

\begin{tabular}{|c|c|c|c|c|c|c|c|c|c|}
\hline $\begin{array}{c}\text { Slte } \\
\text { number }\end{array}$ & $\underset{\text { remarks }}{\text { Design }}$ & $\begin{array}{l}\text { Lead } \\
\text { - partial } \\
\text { (micro- } \\
\text { grams } \\
\text { per gram) }\end{array}$ & $\begin{array}{l}\text { Lithium } \\
\text { (micro- } \\
\text { grams } \\
\text { per gram) }\end{array}$ & $\begin{array}{c}\text { Magneslum } \\
\text { (welght } \\
\text { percent) }\end{array}$ & $\begin{array}{l}\text { Manganese } \\
\text { (mlcro- } \\
\text { grams } \\
\text { per gram) }\end{array}$ & $\begin{array}{l}\text { Mercury } \\
\text { (micro- } \\
\text { grams } \\
\text { per gram) }\end{array}$ & $\begin{array}{c}\text { Molybdenum } \\
\text { (micro- } \\
\text { grams } \\
\text { per gram) }\end{array}$ & $\begin{array}{l}\text { Molybdenum } \\
\text {-partial } \\
\text { (micro- } \\
\text { grams } \\
\text { per gram) }\end{array}$ & $\begin{array}{c}\text { Neodymlum } \\
\text { (micro- } \\
\text { grams } \\
\text { per gram) }\end{array}$ \\
\hline 87 & 1 & 23 & 25 & 2.1 & 990 & 0.21 & $<2$ & 0.84 & 39 \\
\hline 88 & 1 & 15 & 37 & 1.3 & 1,100 & .17 & $<2$ & .87 & 40 \\
\hline 89 & 1 & 9.0 & 31 & 1.3 & 1,800 & .14 & $<2$ & .83 & 80 \\
\hline 89 & 2 & 7.0 & 33 & 1.2 & $1,600^{\circ}$ & .18 & $<2$ & .58 & 50 \\
\hline 89 & 2 & 9.9 & 32 & 1.2 & 1,600 & .16 & $<2$ & .82 & 53 \\
\hline 90 & 1 & 15 & 36 & 1.4 & 2,000 & .17 & $<2$ & .71 & 38 \\
\hline 91 & 1 & 33 & 28 & .97 & 2,100 & .31 & $<2$ & 2.6 & 46 \\
\hline 92 & 1 & 39 & 28 & 1.1 & 2,100 & .41 & $<2$ & 3.1 & 35 \\
\hline 93 & 1 & 26 & 45 & 1.1 & 810 & .09 & $<2$ & .69 & 39 \\
\hline 94 & 1 & 31 & 22 & 3.0 & 3,000 & .44 & 3 & 4.6 & 28 \\
\hline 95 & 1 & 21 & 46 & .97 & 730 & .22 & $<2$ & .76 & 35 \\
\hline 96 & 1 & 25 & 24 & 1.6 & 1,300 & .15 & $<2$ & 2.2 & 30 \\
\hline 97 & 1 & 28 & 28 & 1.5 & 1,400 & .30 & $<2$ & 2.9 & 48 \\
\hline
\end{tabular}


Table 6. Inorganic-constituent concentrations in fine-fraction streambed sediment for tributaries to Lake Champlain--Continued

\begin{tabular}{|c|c|c|c|c|c|c|c|c|c|}
\hline $\begin{array}{c}\text { slto } \\
\text { number }\end{array}$ & $\underset{\text { remarks }}{\text { Deslgn }}$ & $\begin{array}{l}\text { Nlokel } \\
\text { (mloro- } \\
\text { grams } \\
\text { per gram) }\end{array}$ & $\begin{array}{l}\text { Nloblum } \\
\text { (micro- } \\
\text { grams } \\
\text { per gram) }\end{array}$ & $\begin{array}{c}\text { Phosphorus } \\
\text { (weight } \\
\text { percent) }\end{array}$ & $\begin{array}{l}\text { Poteselum } \\
\text { (welght } \\
\text { percent) }\end{array}$ & $\begin{array}{l}\text { Scandlum } \\
\text { (mlcro- } \\
\text { grams } \\
\text { per gram) }\end{array}$ & $\begin{array}{l}\text { Silver } \\
\text { (micro- } \\
\text { grams } \\
\text { por gram) }\end{array}$ & $\begin{array}{l}\text { Silver } \\
\text {-partiai } \\
\text { (mlero- } \\
\text { grams } \\
\text { per gram) }\end{array}$ & $\begin{array}{l}\text { Sodlum } \\
\text { (welght } \\
\text { percent) }\end{array}$ \\
\hline 1 & 1 & 31 & 9 & 0.14 & 1.9 & 11 & $<2$ & 0.07 & 1.7 \\
\hline 1 & 2 & 28 & 10 & .13 & 1.8 & 11 & $<2$ & .08 & 1.7 \\
\hline 1 & 2 & 28 & 9 & .13 & 1.8 & 11 & $<2$ & .10 & 1.8 \\
\hline 4 & 1 & 40 & 10 & .12 & 2.2 & 13 & $<2$ & $<.06$ & 1.5 \\
\hline 4 & 2 & 37 & 11 & .11 & 2.1 & 13 & $<2$ & $<.06$ & 1.7 \\
\hline 4 & 2 & 36 & 11 & .11 & 2.1 & 13 & $<2$ & .07 & 1.6 \\
\hline 5 & 1 & 28 & 13 & .16 & 1.4 & 11 & $<2$ & $<.06$ & 1.7 \\
\hline 6 & 1 & 33 & 4 & .15 & .87 & 5 & 12 & 17 & .60 \\
\hline 8 & 3 & 73 & 12 & .15 & 1.4 & 12 & $<2$ & -- & 1.6 \\
\hline 8 & 1 & 56 & 16 & .16 & 1.4 & 12 & $<2$ & $<.06$ & 1.5 \\
\hline 9 & 2 & 19 & 8 & .13 & 2.1 & 8 & $<2$ & $<.06$ & 1.6 \\
\hline 9 & 1 & 17 & 8 & .12 & 2.0 & 8 & $<2$ & $<.06$ & 1.6 \\
\hline 9 & 2 & 19 & 8 & .13 & 2.1 & 8 & $<2$ & .08 & 1.6 \\
\hline 10 & 1 & 27 & 9 & .18 & 2.3 & 8 & $<2$ & $<.06$ & 1.3 \\
\hline 11 & 2 & 42 & 14 & .13 & 1.6 & 13 & $<2$ & $<.06$ & 1.7 \\
\hline 11 & 1 & 41 & 12 & .13 & 1.6 & 13 & $<2$ & $<.06$ & 1.6 \\
\hline 11 & 2 & 43 & 13 & .13 & 1.5 & 13 & $<2$ & $<.06$ & 1.6 \\
\hline 13 & 1 & 20 & 10 & .15 & 2.0 & 8 & $<2$ & $<.06$ & 1.5 \\
\hline 14 & 1 & 33 & 10 & .15 & 2.1 & 11 & $<2$ & .55 & 1.5 \\
\hline 14 & 3 & 29 & 12 & .15 & 2.2 & 11 & $<2$ & -- & 1.6 \\
\hline 15 & 3 & 60 & 9 & .15 & 2.5 & 11 & $<2$ & -- & 1.3 \\
\hline 16 & 1 & 18 & 9 & .11 & 2.0 & 7 & $<2$ & $<.06$ & 1.8 \\
\hline 18 & 1 & 40 & 11 & .13 & 1.9 & 11 & $<2$ & $<.06$ & 1.5 \\
\hline 19 & 1 & 23 & 7 & .12 & 2.0 & 9 & $<2$ & $<.06$ & 1.5 \\
\hline 20 & 1 & 24 & 12 & .12 & 2.2 & 9 & $<2$ & $<.06$ & 1.5 \\
\hline 21 & 1 & 32 & 7 & .16 & 3.0 & 7 & $<2$ & $<.06$ & .86 \\
\hline 26 & 1 & 39 & 9 & .13 & 2.3 & 7 & $<2$ & .21 & 1.1 \\
\hline 26 & 2 & 34 & 10 & .14 & 2.4 & 7 & $<2$ & .17 & 1.0 \\
\hline 26 & 2 & 34 & 7 & .14 & 2.3 & 7 & $<2$ & .25 & 1.0 \\
\hline 29 & 1 & 27 & 12 & .12 & 1.3 & 10 & $<2$ & $<.06$ & 1.6 \\
\hline 30 & 1 & 26 & 11 & .13 & 2.2 & 9 & $<2$ & $<.06$ & 1.4 \\
\hline 31 & 1 & 35 & 11 & .12 & 1.6 & 12 & $<2$ & $<.06$ & 1.6 \\
\hline 32 & 1 & 23 & 11 & .09 & 2.3 & 7 & $<2$ & $<.06$ & 1.3 \\
\hline 32 & 2 & 22 & 13 & .10 & 2.3 & 7 & $<2$ & -- & 1.2 \\
\hline 32 & 2 & 24 & 4 & .06 & $2 \cdot 3$ & 7 & $<2$ & -- & 1.2 \\
\hline 33 & 1 & 26 & 9 & .11 & 2.1 & 8 & $<2$ & $<.06$ & 1.3 \\
\hline 34 & 1 & 110 & 9 & .11 & 2.3 & 13 & $<2$ & $<.06$ & 1.3 \\
\hline 36 & 1 & 16 & 10 & .13 & 2.2 & 7 & $<2$ & $<.06$ & 1.3 \\
\hline 37 & 1 & 32 & 12 & .12 & 1.6 & 12 & $<2$ & $<.06$ & 1.6 \\
\hline 39 & 1 & 32 & 12 & .11 & 2.0 & 12 & $<2$ & $<.06$ & 1.5 \\
\hline 41 & 1 & 28 & 12 & .11 & 1.4 & 12 & $<2$ & $<.06$ & 1.6 \\
\hline 42 & 1 & 35 & 12 & .14 & 2.1 & 14 & $<2$ & $<.06$ & 1.4 \\
\hline 43 & 1 & 63 & 9 & .13 & 1.9 & 8 & $<2$ & .08 & 1.5 \\
\hline 43 & 2 & 130 & 14 & .13 & 1.8 & 9 & $<2$ & -- & 1.5 \\
\hline
\end{tabular}


Table 6. Inorganic-constituent concentrations in fine-fraction streambed sediment for tributaries to Lake Champlain--Continued

\begin{tabular}{|c|c|c|c|c|c|c|c|c|c|}
\hline $\begin{array}{l}\text { Site } \\
\text { number }\end{array}$ & $\begin{array}{l}\text { Design } \\
\text { remarks }\end{array}$ & $\begin{array}{c}\text { Nickel } \\
\text { (micro- } \\
\text { grams } \\
\text { per gram) }\end{array}$ & $\begin{array}{l}\text { Nioblum } \\
\text { (micro- } \\
\text { grams } \\
\text { per gram) }\end{array}$ & $\begin{array}{l}\text { Phosphorus } \\
\text { (woight } \\
\text { percent) }\end{array}$ & $\begin{array}{c}\text { Potassium } \\
\text { (welght } \\
\text { percent) }\end{array}$ & $\begin{array}{c}\text { Scandium } \\
\text { (micro- } \\
\text { grams } \\
\text { por gram) }\end{array}$ & $\begin{array}{l}\text { Sllver } \\
\text { (micro- } \\
\text { grams } \\
\text { per gram) }\end{array}$ & $\begin{array}{l}\text { Silver } \\
\text {-partial } \\
\text { (micro- } \\
\text { grams } \\
\text { per gram) }\end{array}$ & $\begin{array}{l}\text { Sodium } \\
\text { (welght } \\
\text { percent) }\end{array}$ \\
\hline 43 & 2 & 110 & 15 & 0.13 & 1.7 & 10 & $<2$ & -- & 1.4 \\
\hline 44 & 1 & 32 & 8 & .12 & 1.5 & 12 & $<2$ & .10 & 1.5 \\
\hline 46 & 1 & 65 & 12 & .11 & 1.7 & 13 & $<2$ & .11 & 1.4 \\
\hline 47 & 1 & 15 & 9 & .08 & 2.5 & 6 & $<2$ & $<.06$ & 1.2 \\
\hline 48 & 1 & 33 & 13 & .12 & 1.7 & 11 & $<2$ & $<.06$ & 1.5 \\
\hline 49 & 1 & 20 & 11 & .09 & 2.3 & 7 & $<2$ & $<.06$ & 1.4 \\
\hline 51 & 1 & 29 & 12 & .12 & 2.1 & 10 & $<2$ & $<.06$ & 1.5 \\
\hline 52 & 1 & 32 & 10 & .14 & 2.2 & 11 & $<2$ & $<.06$ & 1.4 \\
\hline 53 & 2 & 28 & 15 & .13 & 1.6 & 12 & $<2$ & $<.06$ & 1.6 \\
\hline 53 & 1 & 26 & 17 & .14 & 1.6 & 13 & $<2$ & $<.06$ & 1.7 \\
\hline 53 & 2 & 28 & 13 & .14 & 1.5 & 12 & $<2$ & $<.06$ & 1.6 \\
\hline 55 & 1 & 50 & 10 & .11 & 2.8 & 15 & $<2$ & $<.06$ & 1.1 \\
\hline 56 & 1 & 40 & 10 & .10 & 2.4 & 13 & $<2$ & $<.06$ & 1.3 \\
\hline 57 & 1 & 29 & 11 & .13 & 1.7 & 10 & $<2$ & $<.06$ & 1.6 \\
\hline 59 & 1 & 57 & 11 & .12 & 2.3 & 11 & $<2$ & $<.06$ & 1.1 \\
\hline 61 & 1 & 22 & 13 & .09 & 1.9 & 11 & $<2$ & $<.06$ & 1.6 \\
\hline 62 & 1 & 27 & 10 & .13 & 2.2 & 12 & $<2$ & .37 & 1.2 \\
\hline 63 & 1 & 32 & 11 & .11 & 1.9 & 12 & $<2$ & $<.06$ & 1.5 \\
\hline 64 & 3 & 35 & 8 & .14 & 1.2 & 10 & $<2$ & -. & 1.5 \\
\hline 65 & 3 & 23 & 11 & .12 & 1.5 & 11 & $<2$ & -- & 1.5 \\
\hline 66 & 1 & 38 & 10 & .13 & 1.6 & 11 & $<2$ & $<.06$ & 1.3 \\
\hline 66 & 3 & 24 & 10 & .13 & 1.5 & 11 & $<2$ & -- & 1.4 \\
\hline 67 & 1 & 39 & 19 & .34 & 1.2 & 15 & $<2$ & $<.06$ & 1.5 \\
\hline 67 & 3 & 23 & 17 & .31 & 1.3 & 13 & $<2$ & -- & 1.5 \\
\hline 68 & 3 & 20 & 18 & .35 & 1.3 & 14 & $<2$ & -- & 1.7 \\
\hline 69 & 1 & 34 & 10 & .15 & 1.6 & 11 & $<2$ & $<.06$ & 1.5 \\
\hline 70 & $1^{\prime}$ & 54 & 10 & .12 & 2.5 & 15 & $<2$ & $<.06$ & 1.0 \\
\hline 71 & 1 & 50 & 12 & .12 & 2.6 & 14 & $<2$ & $<.06$ & 1.1 \\
\hline 72 & 1 & 83 & 12 & .17 & 1.7 & 9 & $<2$ & $<.06$ & 1.2 \\
\hline 73 & 1 & 13 & 12 & .13 & 1.7 & 9 & $<2$ & $<.06$ & 1.4 \\
\hline 75 & 1 & 33 & 9 & .11 & 2.1 & 11 & $<2$ & $<.06$ & 1.2 \\
\hline 76 & 1 & 30 & 9 & .11 & 2.1 & 11 & $<2$ & $<.06$ & 1.3 \\
\hline 77 & 1 & 45 & 9 & .11 & 2.4 & 13 & $<2$ & .09 & .91 \\
\hline 78 & 1 & 32 & 10 & .13 & 1.9 & 10 & $<2$ & .40 & 1.2 \\
\hline 78 & 2 & 34 & 10 & .13 & 2.0 & 10 & $<2$ & .44 & 1.2 \\
\hline 78 & 2 & 33 & 9 & .13 & 1.9 & 10 & $<2$ & .45 & 1.2 \\
\hline 80 & 1 & 40 & 9 & .11 & 2.2 & 12 & $<2$ & $<.06$ & 1.1 \\
\hline 81 & 1 & 45 & 9 & .13 & 2.3 & 14 & $<2$ & $<.06$ & .75 \\
\hline 82 & 1 & 55 & 10 & .10 & 2.5 & 15 & $<2$ & $<.06$ & .88 \\
\hline 83 & 1 & 35 & 9 & .11 & 2.3 & 10 & $<2$ & $<.06$ & 1.0 \\
\hline 84 & 1 & 33 & 7 & .11 & 2.2 & 11 & $<2$ & $<.06$ & 1.2 \\
\hline 85 & 3 & 24 & 6 & .13 & 1.9 & 8 & $<2$ & -- & 1.1 \\
\hline 85 & 1 & 22 & 8 & .11 & 1.9 & 7 & $<2$ & $<.06$ & 1.1 \\
\hline 86 & 1 & 42 & 11 & .13 & 2.5 & 14 & $<2$ & $<.06$ & .87 \\
\hline
\end{tabular}


Table 6. Inorganic-constituent concentrations in fine-fraction streambed sediment for tributaries to Lake Champlain--Continued

\begin{tabular}{|c|c|c|c|c|c|c|c|c|c|}
\hline $\begin{array}{l}\text { Sito } \\
\text { number }\end{array}$ & $\underset{\text { remarks }}{\text { Design }}$ & $\begin{array}{l}\text { Nickel } \\
\text { (micro- } \\
\text { grams } \\
\text { per gram) }\end{array}$ & $\begin{array}{l}\text { Niobium } \\
\text { (micro- } \\
\text { grams } \\
\text { per gram) }\end{array}$ & $\begin{array}{c}\text { Phosphorus } \\
\text { (weight } \\
\text { percent) }\end{array}$ & $\begin{array}{l}\text { Potaeslum } \\
\text { (welght } \\
\text { percent) }\end{array}$ & $\begin{array}{c}\text { Scandlum } \\
\text { (micro- } \\
\text { grams } \\
\text { per gram) }\end{array}$ & $\begin{array}{l}\text { Silver } \\
\text { (micro- } \\
\text { grams } \\
\text { per gram) }\end{array}$ & $\begin{array}{c}\text { Sliver } \\
\text {-partial } \\
\text { (micro- } \\
\text { grams } \\
\text { per gram) }\end{array}$ & $\begin{array}{l}\text { Sodium } \\
\text { (weight } \\
\text { percent) }\end{array}$ \\
\hline 87 & 1 & 33 & 8 & 0.14 & 2.1 & 10 & $<2$ & 0.08 & 1.0 \\
\hline 88 & 1 & 38 & 9 & .09 & 2.3 & 11 & $<2$ & $<.06$ & 1.1 \\
\hline 89 & 1 & 27 & 8 & .12 & 1.7 & 10 & $<2$ & $<.06$ & 1.0 \\
\hline 89 & 2 & 26 & 7 & .11 & 1.7 & 10 & $<2$ & $<.06$ & 1.0 \\
\hline 89 & 2 & 26 & 8 & .11 & 1.7 & 10 & $<2$ & $<.06$ & 1.0 \\
\hline 90 & 1 & 28 & 7 & .12 & 2.0 & 11 & $<2$ & .07 & .99 \\
\hline 91 & 1 & 38 & 8 & .18 & 2.0 & 14 & $<2$ & .07 & .74 \\
\hline 92 & 1 & 62 & 7 & .16 & 2.1 & 12 & $<2$ & .10 & 1.0 \\
\hline 93 & 1 & 40 & 9 & .11 & 2.5 & 14 & $<2$ & $<.06$ & .97 \\
\hline 94 & 1 & 98 & 6 & .14 & 1.6 & 9 & $<2$ & $<.06$ & .94 \\
\hline 95 & 1 & 32 & 5 & .11 & 2.1 & 11 & $<2$ & .13 & 1.2 \\
\hline 96 & 1 & 46 & 7 & .12 & 2.0 & 9 & $<2$ & $<.06$ & 1.1 \\
\hline 97 & 1 & 47 & 7 & .15 & 1.9 & 11 & $<2$ & .07 & 1.2 \\
\hline
\end{tabular}


Table 6. Inorganic-constituent concentrations in fine-fraction streambed sediment for tributaries to Lake Champlain--Continued

\begin{tabular}{|c|c|c|c|c|c|c|c|c|c|}
\hline $\begin{array}{c}\text { Slte } \\
\text { number }\end{array}$ & $\begin{array}{l}\text { Design } \\
\text { remarks }\end{array}$ & $\begin{array}{l}\text { Strontlum } \\
\text { (micro- } \\
\text { grams } \\
\text { per gram) }\end{array}$ & $\begin{array}{l}\text { Tantalum } \\
\text { (mlcro- } \\
\text { grams } \\
\text { por gram) }\end{array}$ & $\begin{array}{l}\text { Thorium } \\
\text { (micro- } \\
\text { grams } \\
\text { per gram) }\end{array}$ & $\begin{array}{c}\text { Tin } \\
\text { (mlcro- } \\
\text { grams } \\
\text { per gram) }\end{array}$ & $\begin{array}{l}\text { Titanlum } \\
\text { (weight } \\
\text { percent) }\end{array}$ & $\begin{array}{l}\text { Uranium } \\
\text { (mlcro- } \\
\text { grams } \\
\text { per gram) }\end{array}$ & $\begin{array}{l}\text { Vanadlum } \\
\text { (mlcro- } \\
\text { grams } \\
\text { per gram) }\end{array}$ & $\begin{array}{l}\text { Ytterblum } \\
\text { (micro- } \\
\text { grams } \\
\text { per gram) }\end{array}$ \\
\hline 1 & 1 & 240 & $<40$ & 7 & $<5$ & 0.50 & $<100$ & 75 & 3 \\
\hline 1 & 2 & 250 & $<40$ & 6 & $<5$ & .48 & $<100$ & 69 & 2 \\
\hline 1 & 2 & 250 & $<40$ & 5 & $<5$ & .48 & $<100$ & 70 & 3 \\
\hline 4 & 1 & 180 & $<40$ & 7 & $<5$ & .46 & $<100$ & 87 & 2 \\
\hline 4 & 2 & 190 & $<40$ & 7 & $<5$ & .51 & $<100$ & 83 & 2 \\
\hline 4 & 2 & 190 & $<40$ & 8 & $<5$ & .49 & $<100$ & 82 & 2 \\
\hline 5 & 1 & 200 & $<40$ & 6 & $<5$ & .75 & $<100$ & 71 & 2 \\
\hline 6 & 1 & 270 & $<40$ & $<4$ & 20 & .15 & $<100$ & 55 & 1 \\
\hline 8 & 3 & 190 & $<40$ & 9 & 8 & .79 & $<100$ & 90 & 3 \\
\hline 8 & 1 & 190 & $<40$ & 9 & 220 & .94 & $<100$ & 87 & 3 \\
\hline 9 & 2 & 240 & $<40$ & 6 & $<5$ & .42 & $<100$ & 50 & 2 \\
\hline 9 & 1 & 240 & $<40$ & $<4$ & $<5$ & .46 & $<100$ & 47 & 2 \\
\hline 9 & 2 & 240 & $<40$ & 6 & $<5$ & .42 & $<100$ & 50 & 3 \\
\hline 10 & 1 & 260 & $<40$ & 6 & 12 & .47 & $<100$ & 53 & 2 \\
\hline 11 & 2 & 200 & $<40$ & 8 & $<5$ & .77 & $<100$ & 81 & 3 \\
\hline 11 & 1 & 190 & $<40$ & 8 & $<5$ & .75 & $<100$ & 82 & 2 \\
\hline 11 & 2 & 200 & $<40$ & 7 & $<5$ & .76 & $<100$ & 79 & 3 \\
\hline 13 & 1 & 340 & $<40$ & 5 & $<5$ & .53 & $<100$ & 49 & 3 \\
\hline 14 & 1 & 200 & $<40$ & 7 & $<5$ & .53 & $<100$ & 77 & 3 \\
\hline 14 & 3 & 210 & $<40$ & 10 & $<5$ & .49 & $<100$ & 76 & 3 \\
\hline 15 & 3 & 170 & $<40$ & 8 & $<5$ & .38 & $<100$ & 87 & 2 \\
\hline 16 & 1 & 300 & $<40$ & $<4$ & $<5$ & .48 & $<100$ & 41 & 3 \\
\hline 18 & 1 & 240 & $<40$ & 6 & $<5$ & .60 & $<100$ & 80 & 2 \\
\hline 19 & 1 & 240 & $<40$ & 6 & $<5$ & .41 & $<100$ & 55 & 3 \\
\hline 20 & 1 & 280 & $<40$ & 7 & $<5$ & .64 & $<100$ & 57 & 3 \\
\hline 21 & 1 & 210 & $<40$ & 5 & $<5$ & .34 & $<100$ & 51 & 3 \\
\hline 26 & 1 & 190 & $<40$ & 7 & 27 & .49 & $<100$ & 56 & 3 \\
\hline 26 & 2 & 190 & $<40$ & 7 & 31 & .56 & $<100$ & 60 & 3 \\
\hline 26 & 2 & 190 & $<40$ & 7 & 30 & .54 & $<100$ & 60 & 3 \\
\hline 29 & 1 & 170 & $<40$ & 8 & $<5$ & .67 & $<100$ & 67 & 2 \\
\hline 30 & 1 & 210 & $<40$ & 8 & $<5$ & .58 & $<100$ & 70 & 4 \\
\hline 31 & 1 & 170 & $<40$ & 8 & $<5$ & .62 & $<100$ & 78 & 2 \\
\hline 32 & 1 & 210 & $<40$ & 9 & $<5$ & .67 & $<100$ & 51 & 4 \\
\hline 32 & 2 & 210 & $<40$ & 10 & $<5$ & .80 & $<100$ & 55 & 4 \\
\hline 32 & 2 & 210 & $<40$ & 8 & $<5$ & .66 & $<100$ & 56 & 4 \\
\hline 33 & 1 & 370 & $<40$ & 6 & $<5$ & .47 & $<100$ & 59 & 3 \\
\hline 34 & 1 & 140 & $<40$ & 7 & 6 & .45 & $<100$ & 89 & 2 \\
\hline 36 & 1 & 200 & $<40$ & 7 & $<5$ & .60 & $<100$ & 47 & 3 \\
\hline 37 & 1 & 170 & $<40$ & 8 & $<5$ & .74 & $<100$ & 78 & 3 \\
\hline 39 & 1 & 170 & $<40$ & 9 & $<5$ & .66 & $<100$ & 79 & 2 \\
\hline 41 & 1 & 160 & $<40$ & 8 & $<5$ & .75 & $<100$ & 73 & 3 \\
\hline 42 & 1 & 150 & $<40$ & 8 & $<5$ & .65 & $<100$ & 90 & 2 \\
\hline 43 & 1 & 220 & $<40$ & 6 & $<5$ & .65 & $<100$ & 53 & 3 \\
\hline 43 & 2 & 220 & $<40$ & 6 & 6 & 1.1 & $<100$ & 73 & 4 \\
\hline 43 & 2 & 210 & $<40$ & 5 & $<5$ & 1.2 & $<100$ & 78 & 4 \\
\hline
\end{tabular}


Table 6. Inorganic-constituent concentrations in fine-fraction streambed sediment for tributaries to Lake Champlain--Continued

\begin{tabular}{|c|c|c|c|c|c|c|c|c|c|}
\hline $\begin{array}{l}\text { Site } \\
\text { number }^{1}\end{array}$ & $\underset{\text { remarks }}{\text { Design }}$ & $\begin{array}{l}\text { Strontium } \\
\text { (mlcro- } \\
\text { grams } \\
\text { per gram) }\end{array}$ & $\begin{array}{c}\text { Tantalum } \\
\text { (mlcro- } \\
\text { grams } \\
\text { per gram) }\end{array}$ & $\begin{array}{l}\text { Thorium } \\
\text { (micro- } \\
\text { grams } \\
\text { per gram) }\end{array}$ & $\begin{array}{c}\text { Tin } \\
\text { (micro- } \\
\text { grams } \\
\text { per gram) }\end{array}$ & $\begin{array}{l}\text { Tltanlum } \\
\text { (weight } \\
\text { percent) }\end{array}$ & $\begin{array}{l}\text { Uranlum } \\
\text { (mlcro- } \\
\text { grams } \\
\text { per gram) }\end{array}$ & $\begin{array}{c}\text { Vanadium } \\
\text { (micro- } \\
\text { grams } \\
\text { per gram) }\end{array}$ & $\begin{array}{c}\text { Ytterblum } \\
\text { (mlcro- } \\
\text { grams } \\
\text { per gram) }\end{array}$ \\
\hline 44 & 1 & 170 & $<40$ & 8 & $<5$ & 0.67 & $<100$ & 77 & 3 \\
\hline 46 & 1 & 170 & $<40$ & 8 & 7 & .74 & $<100$ & 87 & 2 \\
\hline 47 & 1 & 200 & $<40$ & $<4$ & $<5$ & .48 & $<100$ & 42 & 2 \\
\hline 48 & 1 & 170 & $<40$ & 5 & $<5$ & .73 & $<100$ & 73 & 3 \\
\hline 49 & 1 & 230 & $<40$ & 6 & $<5$ & .60 & $<100$ & 51 & 3 \\
\hline 51 & 1 & 200 & $<40$ & 6 & $<5$ & .66 & $<100$ & 69 & 3 \\
\hline 52 & 1 & 200 & $<40$ & 6 & $<5$ & .59 & $<100$ & 73 & 3 \\
\hline 53 & 2 & 260 & $<40$ & 5 & $<5$ & 1.3 & $<100$ & 95 & 5 \\
\hline 53 & 1 & 280 & $<40$ & 5 & $<5$ & 1.6 & $<100$ & 100 & 5 \\
\hline 53 & 2 & 260 & $<40$ & $<4$ & $<5$ & 1.4 & $<100$ & 94 & 4 \\
\hline 55 & 1 & 220 & $<40$ & 11 & $<5$ & .51 & $<100$ & 110 & 3 \\
\hline 56 & 1 & 210 & $<40$ & 10 & $<5$ & .53 & $<100$ & 87 & 3 \\
\hline 57 & 1 & 150 & $<40$ & 7 & $<5$ & .63 & $<100$ & 64 & 3 \\
\hline 59 & 1 & 290 & $<40$ & 7 & $<5$ & .51 & $<100$ & 83 & 3 \\
\hline 61 & 1 & 250 & $<40$ & $<4$ & $<5$ & 1.1 & $<100$ & 80 & 4 \\
\hline 62 & 1 & 120 & $<40$ & 8 & $<5$ & .51 & $<100$ & 72 & 2 \\
\hline 63 & 1 & 240 & $<40$ & 5 & $<5$ & .90 & $<100$ & 83 & 3 \\
\hline 64 & 3 & 280 & $<40$ & 5 & $<5$ & .70 & $<100$ & 77 & 3 \\
\hline 65 & 3 & 260 & $<40$ & 7 & $<5$ & .66 & $<100$ & 77 & 3 \\
\hline 66 & 1 & 240 & $<40$ & 5 & $<5$ & .75 & $<100$ & 82 & 3 \\
\hline 66 & 3 & 270 & $<40$ & 5 & $<5$ & .78 & $<100$ & 85 & 3 \\
\hline 67 & 1 & 200 & $<40$ & 30 & $<5$ & 1.2 & $<100$ & 110 & 16 \\
\hline 67 & 3 & 220 & $<40$ & 25 & $<5$ & .84 & $<100$ & 94 & 14 \\
\hline 68 & 3 & 210 & $<40$ & 29 & $<5$ & .88 & $<100$ & 98 & 17 \\
\hline 69 & 1 & 260 & $<40$ & 8 & $<5$ & .87 & $<100$ & 76 & 5 \\
\hline 70 & 1 & 220 & $<40$ & 11 & $<5$ & .48 & $<100$ & 110 & 3 \\
\hline 71 & 1 & 240 & $<40$ & 10 & $<5$ & .59 & $<100$ & 100 & 2 \\
\hline 72 & 1 & 210 & $<40$ & 7 & $<5$ & .65 & $<100$ & 67 & 5 \\
\hline 73 & 1 & 320 & $<40$ & 7 & $<5$ & .74 & $<100$ & 64 & 3 \\
\hline 75 & 1 & 300 & $<40$ & 7 & $<5$ & .51 & $<100$ & 74 & 2 \\
\hline 76 & 1 & 360 & $<40$ & 6 & $<5$ & .50 & $<100$ & 73 & 3 \\
\hline 77 & 1 & 320 & $<40$ & 8 & $<5$ & .43 & $<100$ & 90 & 3 \\
\hline 78 & 1 & 210 & $<40$ & 6 & 7 & .55 & $<100$ & 72 & 4 \\
\hline 78 & 2 & 200 & $<40$ & 7 & 8 & .59 & $<100$ & 73 & 4 \\
\hline 78 & 2 & 200 & $<40$ & 8 & 12 & .60 & $<100$ & 73 & 4 \\
\hline 80 & 1 & 330 & $<40$ & 9 & $<5$ & .42 & $<100$ & 84 & 2 \\
\hline 81 & 1 & 150 & $<40$ & 10 & $<5$ & .42 & $<100$ & 99 & 2 \\
\hline 82 & 1 & 170 & $<40$ & 12 & $<5$ & .48 & $<100$ & 110 & 3 \\
\hline 83 & 1 & 190 & $<40$ & 8 & $<5$ & .44 & $<100$ & 67 & 3 \\
\hline 84 & 1 & 300 & $<40$ & 7 & $<5$ & .38 & $<100$ & 72 & 2 \\
\hline 85 & 3 & 440 & $<40$ & 7 & $<5$ & .28 & $<100$ & 54 & 2 \\
\hline 85 & 1 & 550 & $<40$ & 6 & $<5$ & .37 & $<100$ & 51 & 2 \\
\hline 86 & 1 & 170 & $<40$ & 9 & $<5$ & .57 & $<100$ & 100 & 3 \\
\hline 87 & 1 & 220 & $<40$ & 7 & $<5$ & .47 & $<100$ & 65 & 3 \\
\hline
\end{tabular}


Table 6. Inorganic-constituent concentrations in fine-fraction streambed sediment for tributaries to Lake Champlain--Continued

\begin{tabular}{|c|c|c|c|c|c|c|c|c|c|}
\hline $\begin{array}{c}\text { Slte } \\
\text { number } 1\end{array}$ & $\underset{\text { remarks }}{\text { Design }}$ & $\begin{array}{l}\text { Strontium } \\
\text { (mlcro- } \\
\text { grams } \\
\text { per gram) }\end{array}$ & $\begin{array}{l}\text { Tantalum } \\
\text { (mlcro- } \\
\text { grams } \\
\text { per gram) }\end{array}$ & $\begin{array}{l}\text { Thorium } \\
\text { (mlcro- } \\
\text { grams } \\
\text { per gram) }\end{array}$ & $\begin{array}{c}\text { Tin } \\
\text { (micro- } \\
\text { grams } \\
\text { per gram) }\end{array}$ & $\begin{array}{l}\text { Titanlum } \\
\text { (wolght } \\
\text { percent) }\end{array}$ & $\begin{array}{l}\text { Uranium } \\
\text { (mlcro- } \\
\text { grams } \\
\text { per gram) }\end{array}$ & $\begin{array}{c}\text { Vanadium } \\
\text { (mlcro- } \\
\text { grams } \\
\text { per gram) }\end{array}$ & $\begin{array}{c}\text { Ytterbium } \\
\text { (mlcro- } \\
\text { grams } \\
\text { per gram) }\end{array}$ \\
\hline 88 & 1 & 150 & $<40$ & 9 & $<5$ & 0.49 & $<100$ & 79 & 2 \\
\hline 89 & 1 & 120 & $<40$ & 16 & $<5$ & .59 & $<100$ & 57 & 2 \\
\hline 89 & 2 & 120 & $<40$ & 10 & $<5$ & .47 & $<100$ & 56 & 2 \\
\hline 89 & 2 & 120 & $<40$ & 10 & $<5$ & .49 & $<100$ & 56 & 2 \\
\hline 90 & 1 & 120 & $<40$ & 8 & $<5$ & .46 & $<100$ & 65 & 2 \\
\hline 91 & 1 & 150 & $<40$ & 7 & $<5$ & .59 & $<100$ & 79 & 5 \\
\hline 92 & 1 & 170 & $<40$ & 6 & $<5$ & .65 & $<100$ & 97 & 3 \\
\hline 93 & 1 & 130 & $<40$ & 10 & $<5$ & .45 & $<100$ & 91 & 2 \\
\hline 94 & 1 & 190 & $<40$ & $<4$ & $<5$ & .44 & $<100$ & 73 & 2 \\
\hline 95 & 1 & 83 & $<40$ & 8 & $<5$ & .29 & $<100$ & 70 & 2 \\
\hline 96 & 1 & 200 & $<40$ & 5 & $<5$ & .41 & $<100$ & 60 & 2 \\
\hline 97 & 1 & 190 & $<40$ & 8 & $<5$ & .51 & $<100$ & 69 & 4 \\
\hline
\end{tabular}


Table 6. Inorganic-constituent concentrations in fine-fraction streambed sediment for tributaries to Lake Champlain--Continued

\begin{tabular}{|c|c|c|c|c|}
\hline $\begin{array}{c}\text { Site } \\
\text { number }\end{array}$ & $\begin{array}{c}\text { Design } \\
\text { remarks }\end{array}$ & $\begin{array}{l}\text { Yttrium } \\
\text { (micro- } \\
\text { grams } \\
\text { per gram) }\end{array}$ & $\begin{array}{c}\text { Znc } \\
\text { (micro- } \\
\text { grams } \\
\text { per gram) }\end{array}$ & $\begin{array}{c}\text { Znc } \\
\text {-partial } \\
\text { (mlcro- } \\
\text { grams } \\
\text { per gram) }\end{array}$ \\
\hline 1 & 1 & 29 & 110 & 120 \\
\hline 1 & 2 & 29 & 110 & 110 \\
\hline 1 & 2 & 29 & 110 & 100 \\
\hline 4 & 1 & 25 & 100 & 100 \\
\hline 4 & 2 & 26 & 92 & 90 \\
\hline 4 & 2 & 26 & 92 & 74 \\
\hline 5 & 1 & 29 & 89 & 82 \\
\hline 6 & 1 & 12 & 400 & 320 \\
\hline 8 & 3 & 28 & 120 & -- \\
\hline 8 & 1 & 32 & 130 & 120 \\
\hline 9 & 2 & 29 & 89 & 87 \\
\hline 9 & 1 & 28 & 76 & 74 \\
\hline 9 & 2 & 29 & 90 & 90 \\
\hline 10 & 1 & 28 & 110 & 110 \\
\hline 11 & 2 & 33 & 87 & 61 \\
\hline 11 & 1 & 30 & 91 & 84 \\
\hline 11 & 2 & 30 & 83 & 77 \\
\hline 13 & 1 & 29 & 79 & 77 \\
\hline 14 & 1 & 30 & 140 & 140 \\
\hline 14 & 3 & 27 & 130 & -- \\
\hline 15 & 3 & 23 & 180 & -- \\
\hline 16 & 1 & 27 & 53 & 48 \\
\hline 18 & 1 & 29 & 88 & 86 \\
\hline 19 & 1 & 29 & 120 & 130 \\
\hline 20 & 1 & 32 & 71 & 66 \\
\hline 21 & 1 & 27 & 99 & 77 \\
\hline 26 & 1 & 31 & 160 & 140 \\
\hline 26 & 2 & 33 & 190 & 160 \\
\hline 26 & 2 & 32 & 180 & 190 \\
\hline 29 & 1 & 27 & 59 & 53 \\
\hline 30 & 1 & 47 & 160 & 140 \\
\hline 31 & 1 & 29 & 67 & 57 \\
\hline 32 & 1 & 36 & 43 & 25 \\
\hline 32 & 2 & 39 & 40 & -- \\
\hline 32 & 2 & 35 & 44 & -- \\
\hline 33 & 1 & 28 & 79 & 80 \\
\hline 34 & 1 & 28 & 110 & 110 \\
\hline 36 & 1 & 33 & 47 & 29 \\
\hline 37 & 1 & 31 & 75 & 67 \\
\hline 39 & 1 & 30 & 77 & 74 \\
\hline 41 & 1 & 31 & 66 & 61 \\
\hline 42 & 1 & 30 & 110 & 100 \\
\hline 43 & 1 & 39 & 100 & 80 \\
\hline 43 & 2 & 46 & 110 & $\cdots$ \\
\hline
\end{tabular}


Table 6. Inorganic-constituent concentrations in fine-fraction streambed sediment for tributaries to Lake Champlain--Continued

\begin{tabular}{|c|c|c|c|c|}
\hline $\begin{array}{c}\text { Site } \\
\text { number } 1\end{array}$ & $\underset{\text { remarks }}{\text { Design }}$ & $\begin{array}{c}\text { Yttrium } \\
\text { (mlcro- } \\
\text { grams } \\
\text { per gram) }\end{array}$ & $\begin{array}{c}\text { Zinc } \\
\text { (mlcro- } \\
\text { grams } \\
\text { per gram) }\end{array}$ & $\begin{array}{c}\text { Zinc } \\
\text {-partlal } \\
\text { (mlcro- } \\
\text { grams } \\
\text { per gram) }\end{array}$ \\
\hline 43 & 2 & 47 & 110 & $\ldots$ \\
\hline 44 & 1 & 31 & 79 & 77 \\
\hline 46 & 1 & 29 & 100 & 98 \\
\hline 47 & 1 & 23 & 43 & 29 \\
\hline 48 & 1 & 30 & 140 & 140 \\
\hline 49 & 1 & 27 & 45 & 29 \\
\hline 51 & 1 & 30 & 82 & 77 \\
\hline 52 & 1 & 30 & 77 & 78 \\
\hline 53 & 2 & 50 & 89 & 53 \\
\hline 53 & 1 & 55 & 75 & 35 \\
\hline 53 & 2 & 49 & 86 & 61 \\
\hline 55 & 1 & 29 & 110 & 120 \\
\hline 56 & 1 & 31 & 84 & 91 \\
\hline 57 & 1 & 31 & 68 & 67 \\
\hline 59 & 1 & 27 & 76 & 81 \\
\hline 61 & 1 & 41 & 76 & 61 \\
\hline 62 & 1 & 27 & 100 & 110 \\
\hline 63 & 1 & 39 & 85 & 72 \\
\hline 64 & 3 & 32 & 92 & -- \\
\hline 65 & 3 & 34 & 80 & -- \\
\hline 66 & 1 & 37 & 87 & 77 \\
\hline 66 & 3 & 34 & 81 & - \\
\hline 67 & 1 & 200 & 140 & 120 \\
\hline 67 & 3 & 150 & 130 & $\ldots$ \\
\hline 68 & 3 & 180 & 110 & -- \\
\hline 69 & 1 & 60 & 100 & 87 \\
\hline 70 & 1 & 32 & 100 & 110 \\
\hline 71 & 1 & 29 & 100 & 110 \\
\hline 72 & 1 & 60 & 94 & 87 \\
\hline 73 & 1 & 37 & 56 & 48 \\
\hline 75 & 1 & 28 & 81 & 83 \\
\hline 76 & 1 & 30 & 75 & 72 \\
\hline 77 & 1 & 28 & 92 & 95 \\
\hline 78 & 1 & 42 & 170 & 180 \\
\hline 78 & 2 & 44 & 170 & 170 \\
\hline 78 & 2 & 45 & 160 & 170 \\
\hline 80 & 1 & 29 & 81 & 86 \\
\hline 81 & 1 & 30 & 140 & 140 \\
\hline 82 & 1 & 35 & 110 & 120 \\
\hline 83 & 1 & 29 & 74 & 76 \\
\hline 84 & 1 & 27 & 81 & 82 \\
\hline 85 & 3 & 23 & 69 & -- \\
\hline 85 & 1 & 24 & 65 & 54 \\
\hline 86 & 1 & 29 & 110 & 110 \\
\hline
\end{tabular}


Table 6. Inorganic-constituent concentrations in fine-fraction streambed sediment for tributaries to Lake Champlain-Continued

\begin{tabular}{|c|c|c|c|c|}
\hline $\begin{array}{l}\text { Sto } \\
\text { number } 1\end{array}$ & $\begin{array}{l}\text { Dowign } \\
\text { remartios }\end{array}$ & $\begin{array}{l}\text { Ytulum } \\
\text { (mloro- } \\
\text { grams } \\
\text { per grem) }\end{array}$ & $\begin{array}{l}\text { Zinc } \\
\text { (micro- } \\
\text { grams } \\
\text { per grem) }\end{array}$ & $\begin{array}{l}\text { Zne } \\
\text {-partiai } \\
\text { (mloro- } \\
\text { grams } \\
\text { per gram) }\end{array}$ \\
\hline 87 & 1 & 41 & 110 & 84 \\
\hline 88 & 1 & 27 & 73 & 77 \\
\hline 89 & 1 & 25 & 67 & 57 \\
\hline 89 & 2 & 22 & 68 & 45 \\
\hline 89 & 2 & 22 & 68 & 60 \\
\hline 90 & 1 & 22 & 79 & 76 \\
\hline 91 & 1 & 57 & 200 & 190 \\
\hline 92 & 1 & 42 & 220 & 210 \\
\hline 93 & 1 & 28 & 110 & 110 \\
\hline 94 & 1 & 30 & 420 & 360 \\
\hline 95 & 1 & 18 & 110 & 100 \\
\hline 96 & 1 & 29 & 290 & 300 \\
\hline 97 & 1 & 50 & 160 & 170 \\
\hline
\end{tabular}

${ }^{1}$ Location of site number shown in figure 1.

${ }^{2}$ Remark code 1 means a normal sample used in calculation of summary statistics; 2 means same-day resampling of site used for nested ANOVA, resampled material was split at the laboratory so that two samples were analyzed; 3 means site was sampled during follow-up investigation in October 1992. 
Table 7. Domain, international Unlon of Practical and Applled Chemlstry (IUPAC) structure number, and name of polychlorinated blphenyl congeners in order of elution from the gas chromatograph

[IUPAC, International Union of Practical and Applied Chemistry]

\begin{tabular}{lll}
\hline Domain & $\begin{array}{l}\text { IUPAC } \\
\text { number }\end{array}$ Congener name \\
\hline
\end{tabular}

\begin{tabular}{|c|c|c|}
\hline 1 & 10 & 2,6-Dichlorobiphenyl \\
\hline 1 & 4 & $2,2^{\prime}$-Dichlorobiphenyl \\
\hline 2 & 7 & 2,4-Dichlorobiphenyl \\
\hline 2 & 9 & 2,5-Dichlorobiphenyl \\
\hline 3 & 6 & $2,3^{\prime}$-Dichlorobiphenyl \\
\hline 4 & 8 & 2,4'-Dichlorobiphenyl \\
\hline 4 & 5 & 2,3-Dichlorobiphenyl \\
\hline 5 & 19 & $2,2^{\prime}, 6$-Trichlorobiphenyl \\
\hline 6 & 18 & $2,2^{\prime}, 5-\operatorname{Tr}$ ichlorobipheny 1 \\
\hline 7 & 15 & 4,4'-Dichlorobiphenyl \\
\hline 7 & 17 & $2,2^{\prime}, 4$-Trichlorobipheny 1 \\
\hline 8 & 24 & $2,3,6$-Trichlorobiphenyl \\
\hline 8 & 27 & $2,3^{\prime}, 6-\operatorname{Trich}$ lorobiphenyl \\
\hline 9 & 16 & $2,2^{\prime}, 3-\operatorname{Trich}$ lorobiphenyl \\
\hline 9 & 32 & $2,4^{\prime}, 6$-Trichlorobiphenyl \\
\hline 10 & 26 & $2,3^{\prime}, 5$-Trichlorobiphenyl \\
\hline 11 & 25 & $2,3^{\prime}, 4-\operatorname{Tr}$ ichlorobipheny 1 \\
\hline 12 & 31 & $2,4^{\prime}, 5$-Trichlorobiphenyl \\
\hline 12 & 28 & $2,4,4^{\prime}$-Trichlorobiphenyl \\
\hline 13 & 33 & $2^{\prime}, 3,4-\operatorname{Tr}$ ichlorobiphenyl \\
\hline 13 & 20 & $2,3,3^{\prime}$-Trichlorobiphenyl \\
\hline 13 & 53 & $2,2^{\prime}, 5,6^{\prime}$-Tetrachlorobiphenyl \\
\hline 14 & 51 & $2,2^{\prime}, 4,6^{\prime}$-Tetrachlorobiphenyl \\
\hline 14 & 22 & $2,3,4^{\prime}-\operatorname{Tr}$ ichlorobiphenyl \\
\hline 15 & 45 & $2,2^{\prime}, 3,6$-Tetrachlorobiphenyl \\
\hline 16 & 46 & $2,2^{\prime}, 3,6^{\prime}$-Tetrachlorobiphenyl \\
\hline 17 & 52 & $2,2^{\prime}, 5,5^{\prime}$-Tetrachlorobipheny 1 \\
\hline 18 & 49 & $2,2^{\prime}, 4,5^{\prime}$-Tetrachlorobiphenyl \\
\hline 19 & 47 & $2,2^{\prime} 4,4^{\prime}$-Tetrachlorobiphenyl \\
\hline 19 & 48 & $2,2^{\prime}, 4,5$-Tetrachlorobiphenyl \\
\hline 19 & 75 & $2,4,4^{\prime}, 6$-Tetrachlorobiphenyl \\
\hline 20 & 44 & $2,2^{\prime}, 3,5^{\prime}$-Tetrachlorobipheny 1 \\
\hline 21 & 59 & $2,3,3^{\prime}, 6$-Tetrachlorobipheny 1 \\
\hline 21 & 42 & $2,2^{\prime}, 3,4^{\prime}$-Tetrachlorobiphenyl \\
\hline 21 & 37 & $3,4,4^{\prime}-\operatorname{Tr}$ ichlorobipheny 1 \\
\hline 22 & 41 & $2,2 ', 3,4$-Tetrachlorobipheny 1 \\
\hline 22 & 64 & $2,3,4^{\prime}, 6$-Tetrachlorobipheny 1 \\
\hline 23 & 40 & $2,2^{\prime}, 3,3^{\prime}$-Tetrachlorobipheny 1 \\
\hline 24 & 74 & $2,4,4,, 5$-Tetrachlorobiphenyl \\
\hline 25 & 91 & $2,2^{\prime}, 3,4^{\prime}, 6$-Pentachlorobipheny \\
\hline
\end{tabular}


Table 7. Domain, internationai Union of Practical and Applied Chemlstry (IUPAC) structure number, and name of polychlorinated blphenyl congeners in order of elution from the gas chromatograph-Continued

\begin{tabular}{lll}
\hline Domain & $\begin{array}{l}\text { lupAC } \\
\text { number }\end{array}$ & Congener name \\
\hline
\end{tabular}

\begin{tabular}{|c|c|c|}
\hline 26 & 60 & $2,3,4,4^{\prime}$-Tetrachlorobipheny 1 \\
\hline 26 & 56 & $2,3,3^{\prime}, 4^{\prime}$-Tetrachlorobipheny 1 \\
\hline 26 & 92 & $2,2^{\prime}, 3,5,5^{\prime}$-Pentachlorobiphenyl \\
\hline 27 & 84 & $2,2^{\prime}, 3,3^{\prime}, 6$-Pentachlorobiphenyl \\
\hline 28 & 90 & $2,2^{\prime}, 3,4^{\prime}, 5$-Pentachlorobipheny 1 \\
\hline 28 & 101 & $2,2^{\prime}, 4,5,5^{\prime}$-Pentachlorobipheny 1 \\
\hline 29 & 99 & $2,2^{\prime}, 4,4^{\prime}, 5$-Pentachlorobiphenyl \\
\hline 30 & 83 & $2,2^{\prime}, 3,3^{\prime}, 5$-Pentachlorobiphenyl \\
\hline 31 & 97 & $2,2^{\prime}, 3^{\prime}, 4,5$-Pentachlorobipheny 1 \\
\hline 32 & 87 & $2,2^{\prime}, 3,4,5^{\prime}$-Pentachlorobipheny 1 \\
\hline 32 & 115 & $2,3,4,4^{\prime}, 6$-Pentachlorobipheny 1 \\
\hline 33 & 85 & $2,2^{\prime}, 3,4,4^{\prime}$-Pentachlorobiphenyl \\
\hline 34 & 136 & $2,2^{\prime}, 3,3^{\prime}, 6,6^{\prime}$-Hexachlorobipheny 1 \\
\hline 35 & 77 & $3,3^{\prime}, 4,4^{\prime}$-Tetrachlorobiphenyl \\
\hline 35 & 110 & $2,3,3^{\prime}, 4^{\prime}, 6$-Pentachlorobiphenyl \\
\hline 36 & 82 & $2,2^{\prime}, 3,3^{\prime}, 4$-Pentachlorobipheny 1 \\
\hline 36 & 151 & $2,2^{\prime}, 3,5,5^{\prime}, 6$-Hexachlorobiphenyl \\
\hline 37 & 135 & $2,2^{\prime}, 3,3^{\prime}, 5,6^{\prime}$-Hexachlorobipheny 1 \\
\hline 38 & 123 & $2^{\prime}, 3,4,4^{\prime}, 5$-Pentachlorobiphenyl \\
\hline 38 & 149 & $2,2^{\prime}, 3,4^{\prime}, 5^{\prime}, 6$-Hexachlorobiphenyl \\
\hline 38 & 118 & $2,3^{\prime}, 4,4^{\prime}, 5$-Pentachlorobiphenyl \\
\hline 39 & 134 & $2,2^{\prime}, 3,3^{\prime}, 5,6$-Hexachlorobiphenyl \\
\hline 40 & 114 & $2,3,4,4^{\prime}, 5$-Pentachlorobiphenyl \\
\hline 40 & 131 & $2,2^{\prime}, 3,3^{\prime}, 4,6$-Hexachlorobipheny 1 \\
\hline 40 & 122 & $2^{\prime}, 3,3^{\prime} 4,5$-Pentachlorobipheny 1 \\
\hline 41 & 146 & $2,2^{\prime}, 3,4^{\prime}, 5,5^{\prime}$-Hexachlorobiphenyl \\
\hline 42 & 132 & $2,2^{\prime}, 3,3^{\prime}, 4,6^{\prime}$-Hexachlorobipheny 1 \\
\hline 42 & 153 & $2,2^{\prime}, 4,4^{\prime}, 5,5^{\prime}$-Hexachlorobipheny 1 \\
\hline 42 & 105 & $2,3,3^{\prime}, 4,4^{\prime}$-Pentachlorobiphenyl \\
\hline 43 & 141 & $2,2^{\prime}, 3,4,5,5^{\prime}$-Hexachlorobipheny 1 \\
\hline 43 & 179 & $2,2^{\prime}, 3,3^{\prime}, 5,6,6^{\prime}$-Heptachlorobiphenyl \\
\hline 44 & 176 & $2,2^{\prime}, 3,3^{\prime}, 4,6,6^{\prime}$-Heptachlorobiphenyl \\
\hline 44 & 137 & $2,2^{\prime}, 3,4,4^{\prime}, 5$-Hexachlorobipheny 1 \\
\hline 45 & 138 & $2,2^{\prime}, 3,4,4^{\prime}, 5^{\prime}-$ Hexachlorobipheny 1 \\
\hline 45 & 160 & $2,3,3^{\prime}, 4,5,6$-Hexachlorobipheny 1 \\
\hline 46 & 158 & $2,3,3^{\prime}, 4,4^{\prime}, 6$-Hexachlorobiphenyl \\
\hline 47 & 129 & $2,2^{\prime}, 3,3^{\prime}, 4,5$-Hexachlorobipheny 1 \\
\hline 47 & 126 & $3,3^{\prime}, 4,4^{\prime}, 5$-Pentachlorobipheny 1 \\
\hline 47 & 178 & $2,2^{\prime}, 3,3^{\prime}, 5,5^{\prime}, 6$-Heptachlorobiphenyl \\
\hline 48 & 175 & $2,2^{\prime}, 3,3^{\prime}, 4,5^{\prime}, 6$-Heptachlorobipheny 1 \\
\hline
\end{tabular}


Table 7. Domain, International Union of Practical and Applied Chemistry (IUPAC) structure number, and name of polychlorinated blphenyl congeners in order of elution from the gas chromatograph -Continued

\begin{tabular}{|c|c|c|}
\hline Domain & $\begin{array}{l}\text { IUPAC } \\
\text { number }\end{array}$ & Congener name \\
\hline 49 & 187 & $2,2^{\prime}, 3,4^{\prime}, 5,5^{\prime}, 6$-Heptachlorobiphenyl \\
\hline 50 & 183 & $2,2^{\prime}, 3,4,4^{\prime}, 5^{\prime}, 6$-Heptachlorobiphenyl \\
\hline 51 & 128 & $2,2^{\prime}, 3,3^{\prime}, 4,4^{\prime}$-Hexachlorobiphenyl \\
\hline 52 & 185 & $2,2^{\prime}, 3,4,5,5^{\prime}, 6$-Heptachlorobipheny 1 \\
\hline 53 & 174 & $2,2^{\prime}, 3,3^{\prime}, 4,5,6^{\prime}$-Heptachlorobipheny 1 \\
\hline 54 & 177 & $2,2^{\prime}, 3,3^{\prime}, 4^{\prime}, 5,6$-Heptachlorobiphenyl \\
\hline 55 & 202 & $2,2^{\prime}, 3,3^{\prime}, 5,5^{\prime}, 6,6^{\prime}$-octachlorobiphenyl \\
\hline 55 & 171 & $2,2^{\prime}, 3,3^{\prime}, 4,4^{\prime}, 6$-Heptachlorobipheny 1 \\
\hline 55 & 156 & $2,3,3^{\prime}, 4,4^{\prime}, 5$-Hexachlorobiphenyl \\
\hline 56 & 173 & $2,2^{\prime}, 3,3,4,5,6$-Heptachlorobipheny 1 \\
\hline 56 & 157 & $2,3,3^{\prime}, 4,4^{\prime}, 5^{\prime}$-Hexachlorobipheny 1 \\
\hline 56 & 201 & $2,2^{\prime}, 3,3^{\prime}, 4,5^{\prime}, 6,6^{\prime}$-Octachlorobiphenyl \\
\hline 57 & 172 & $2,2^{\prime}, 3,3^{\prime}, 4,5,5^{\prime}$-Heptachlorobiphenyl \\
\hline 58 & 180 & $2,2^{\prime}, 3,4,4^{\prime}, 5,5^{\prime}$-Heptachlorobiphenyl \\
\hline 59 & 193 & $2,3,3^{\prime}, 4^{\prime}, 5,5^{\prime}, 6$-Heptachlorobipheny 1 \\
\hline 60 & 200 & $2,2^{\prime}, 3,3^{\prime}, 4,5,6,6^{\prime}$-Octachlorobiphenyl \\
\hline 61 & 170 & $2,2^{\prime}, 3,3^{\prime}, 4,4^{\prime}, 5$-Heptachlorobiphenyl \\
\hline 61 & 190 & $2,3,3^{\prime}, 4,4^{\prime}, 5,6$-Heptachlorobipheny 1 \\
\hline 62 & 198 & $2,2^{\prime}, 3,3^{\prime}, 4,5,5^{\prime}, 6$-Octachlorobiphenyl \\
\hline 63 & 203 & $2,2^{\prime}, 3,4,4^{\prime}, 5,5^{\prime}, 6$-octachlorobiphenyl \\
\hline 63 & 196 & $2,2^{\prime}, 3,3^{\prime}, 4,4^{\prime}, 5^{\prime}, 6$-Octachlorobipheny 1 \\
\hline 64 & 208 & $2,2^{\prime}, 3,3^{\prime}, 4,5,5^{\prime}, 6,6^{\prime}$-Nonachlorobiphenyl \\
\hline 64 & 195 & $2,2^{\prime}, 3,3^{\prime}, 4,4^{\prime}, 5,6$-octachlorobipheny 1 \\
\hline 65 & 194 & $2,2^{\prime}, 3,3^{\prime}, 4,4^{\prime}, 5,5^{\prime}$-octachlorobiphenyl \\
\hline 66 & 205 & $2,3,3^{\prime}, 4,4^{\prime}, 5,5^{\prime}, 6$-octachlorobiphenyl \\
\hline
\end{tabular}


Table 8. Comparison of polychlorinated blphenyl congener standard-reference material HS-2 to analytical results

[All concentrations are in nanograms per gram; IUPAC, International Union of Practical and Applied Chemistry; values in parenthesis are 2 standard deviations]

\begin{tabular}{|c|c|c|c|c|c|}
\hline \multirow{2}{*}{$\begin{array}{l}\text { IUPAC } \\
\text { Structure } \\
\text { number }\end{array}$} & \multirow[b]{2}{*}{ Congener name } & \multirow{2}{*}{\multicolumn{2}{|c|}{$\begin{array}{l}\text { Standard concentration } \\
\text { of reference material HS-2 }\end{array}$}} & \multicolumn{2}{|c|}{ Laboratory concentration } \\
\hline & & & & Sample1 & Sample2 \\
\hline 180 & $2,2^{\prime}, 3,4,4^{\prime}, 5,5^{\prime}$-Heptachlorobiphenyl & 3.70 & $(0.33)$ & 5.5 & 5.5 \\
\hline 194 & $2,2^{\prime}, 3,3^{\prime}, 4,4^{\prime}, 5^{\prime} 5^{\prime}$-Octachlorobipheny 1 & .61 & $(.07)$ & 2.0 & .8 \\
\hline
\end{tabular}


Table 9. Carbonate, organic, and total carbon concentrations, and polychlorinated biphenyl compound detections in streambed sediment for tributaries to Lake Champlain [--, data not available; <, less than reporting level given; PCB's, polychlorinated biphenyl congeners]

\begin{tabular}{|c|c|c|c|c|c|c|c|c|c|}
\hline $\begin{array}{l}\text { Site } \\
\text { num- } \\
\text { ber' }^{1}\end{array}$ & Stream name & $\begin{array}{l}\text { sign } \\
\text { rks }^{2}\end{array}$ & Date & $\begin{array}{l}\text { Specific } \\
\text { conduct- } \\
\text { ance } \\
\text { (micro- } \\
\text { siemens } \\
\text { per centi- } \\
\text { meter) }\end{array}$ & $\begin{array}{l}\text { pH } \\
\text { (stand- } \\
\text { ard } \\
\text { units }\end{array}$ & $\begin{array}{l}\text { Carbon- } \\
\text { ate } \\
\text { carbon } \\
\text { (per- } \\
\text { cent) }\end{array}$ & $\begin{array}{l}\text { Organic } \\
\text { carbon } \\
\text { (per } \\
\text { cent) }\end{array}$ & $\begin{array}{l}\text { Total } \\
\text { carbon } \\
\text { (per- } \\
\text { cent) }\end{array}$ & $\begin{array}{l}\text { PCB's } \\
\text { de- } \\
\text { tected }^{3}\end{array}$ \\
\hline 1 & Pike River & 1 & $7-21-92$ & 389 & 8.7 & 0.02 & 0.94 & 0.96 & no \\
\hline 1 & Pike River & 2 & $7-21-92$ & 389 & 8.7 & .01 & .89 & .90 & no \\
\hline 2 & Lake Bed near Pike River & 1 & $6-29-92$ & -- & -- & .02 & 2.87 & 2.89 & yes \\
\hline 2 & Lake Bed near pike River & 2 & $6-29-92$ & -- & -- & .03 & 2.87 & 2.90 & yes \\
\hline 3 & Lake Bed near Missisquoi River & 1 & $6-29-92$ & -- & -- & .02 & 3.26 & 3.28 & yes \\
\hline 3 & Lake Bed near Missisquoi River & 1 & $6-29-92$ & -- & -- & .02 & 3.26 & 3.28 & no \\
\hline 3 & Lake Bed near Missisquoi River & 2 & $6-29-92$ & -- & -- & .07 & 3.06 & 3.13 & yes \\
\hline 4 & Rock River & 1 & $7-02-92$ & 363 & 7.3 & .02 & 1.99 & 2.01 & no \\
\hline 7 & Mud Creek & 1 & $7-22-92$ & 434 & 7.0 & .05 & 8.21 & 8.26 & yes \\
\hline 9 & Great Chazy River & 1 & $7-22-92$ & 198 & 7.2 & .02 & .65 & .67 & no \\
\hline 9 & Great Chazy River & 2 & $7-22-92$ & 198 & 7.2 & .03 & .71 & .74 & no \\
\hline 11 & Missisquoi River & 1 & $7-02-92$ & 150 & 7.5 & $<.01$ & .85 & .85 & no \\
\hline 12 & Lake Bed near Great Chazy River & 1 & $6-29-92$ & -- & -- & .14 & .77 & .91 & no \\
\hline 13 & Little Chazy River & 1 & $7-22-92$ & 256 & 8.8 & .78 & .41 & 1.19 & no \\
\hline 14 & Stephens Brook & 1 & $7-02-92$ & 704 & 8.2 & .05 & .70 & .75 & yes \\
\hline 16 & Riley Brook & 1 & $7-23-92$ & 649 & 7.2 & .63 & 1.22 & 1.85 & no \\
\hline 17 & Lake Bed in St. Albans Bay & 1 & $6-23-92$ & -- & -- & .01 & 5.69 & 5.70 & yes \\
\hline 18 & Mill River & 1 & $6-04-92$ & 479 & 8.5 & -- & -- & -- & no \\
\hline 18 & Mill River & 2 & $6-04-92$ & 479 & 8.5 & -- & -- & -- & no \\
\hline 19 & Dead Creek & 1 & $7-23-92$ & 417 & 7.3 & .06 & 3.95 & 4.01 & yes \\
\hline 22 & Dead Creek Marsh & 1 & $7-28-92$ & 450 & 7.4 & .092 & 28.70 & 28.80 & yes \\
\hline 23 & unnamed tributary & 1 & $8-11-92$ & -- & -- & .75 & .25 & 1.00 & yes \\
\hline 24 & Lake Bed in Cumberland Bay & 1 & $7-28-92$ & -- & -- & .06 & 8.98 & 9.04 & yes \\
\hline 24 & Lake Bed in Cumberland Bay & 1 & $7-28-92$ & -- & -- & .06 & 8.98 & 9.04 & Yes \\
\hline 24 & Lake Bed in Cumberland Bay & 2 & $7-28-92$ & -- & -- & $<.01$ & 9.64 & 9.64 & yes \\
\hline 25 & Lake Bed in Cumberland Bay & 1 & $6-23-92$ & -- & -- & .01 & 3.68 & 3.69 & Yes \\
\hline 26 & Saranac River & 1 & $7-28-92$ & 93 & 7.2 & .11 & .88 & .99 & yes \\
\hline 26 & Saranac River & 2 & $7-28-92$ & 93 & 7.2 & .10 & .96 & 1.06 & yes \\
\hline 27 & Saranac River & 1 & $7-28-92$ & 85 & 7.2 & .04 & .18 & .22 & no \\
\hline 28 & Saranac River & 1 & $7-28-92$ & 85 & 7.2 & .03 & .26 & .29 & no \\
\hline 29 & Stone Bridge Brook & 1 & $7-06-92$ & 278 & 7.6 & $<.01$ & .14 & .14 & no \\
\hline 30 & unnamed tributary & 1 & $7-29-92$ & 530 & 7.4 & .03 & .23 & .26 & yes \\
\hline 32 & Salmon River & 1 & $7-29-92$ & 192 & 7.2 & .18 & .13 & .31 & yes \\
\hline 32 & Salmon River & 2 & $7-29-92$ & 192 & 7.2 & .12 & .09 & .21 & Yes \\
\hline 32 & Salmon River & 2 & $7-29-92$ & 192 & 7.2 & .12 & .09 & .21 & yes \\
\hline 34 & Lamoille River & 1 & $7-02-92$ & -- & 6.7 & $<.01$ & .14 & .14 & no \\
\hline 35 & Lake Bed in Malletts Bay & 1 & $6-23-92$ & -- & -- & .03 & 2.74 & 2.77 & no \\
\hline 36 & Little Ausable River & 1 & $7-29-92$ & 251 & 7.4 & .10 & 1.24 & 1.34 & no \\
\hline 38 & Lake Bed in Malletts Bay & 1 & $6-23-92$ & -- & -- & .02 & 3.90 & 3.92 & yes \\
\hline 38 & Lake Bed in Malletts Bay & 1 & $6-23-92$ & -- & -- & .02 & 3.90 & 3.92 & Yes \\
\hline
\end{tabular}




\begin{tabular}{|c|c|c|c|c|c|c|c|c|c|}
\hline $\begin{array}{l}\text { Site } \\
\text { num- } \\
\text { ber } 1\end{array}$ & Stream name & $\begin{array}{l}\text { Design } \\
\text { re- } \\
\text { narks } 2\end{array}$ & Date & $\begin{array}{l}\text { Specific } \\
\text { conduct- } \\
\text { ance } \\
\text { (micro- } \\
\text { slemens } \\
\text { per centi- } \\
\text { meter) }\end{array}$ & $\begin{array}{l}\text { pH } \\
\text { (stand- } \\
\text { ard } \\
\text { units }\end{array}$ & $\begin{array}{l}\text { Carbon- } \\
\text { ate } \\
\text { carbon } \\
\text { (per- } \\
\text { cent) }\end{array}$ & $\begin{array}{l}\text { Organic } \\
\text { carbon } \\
\text { (per } \\
\text { cent) }\end{array}$ & $\begin{array}{l}\text { Total } \\
\text { carbon } \\
\text { (per- } \\
\text { cent) }\end{array}$ & $\begin{array}{l}\text { PCB's } \\
\text { de- } \\
\text { tected }\end{array}$ \\
\hline 39 & Malletts Creek & 1 & $7-06-92$ & 313 & 8.0 & 0.04 & 0.43 & 0.47 & yes \\
\hline 39 & Malletts Creek & 1 & $7-06-92$ & 313 & 8.0 & .04 & .43 & .47 & yes \\
\hline 40 & Lake bed near Ausable River & 1 & $6-24-92$ & -- & -- & .03 & 5.62 & 5.65 & yes \\
\hline 41 & Indian Brook & 1 & $7-06-92$ & 493 & 7.6 & $<.01$ & .27 & .27 & Yes \\
\hline 43 & Ausable River & 1 & $7-27-92$ & 97 & 7.8 & $<.01$ & .09 & .09 & no \\
\hline 43 & Ausable River & 2 & $7-27-92$ & 97 & 7.8 & .02 & .27 & .29 & no \\
\hline 44 & Winooski River & 1 & $7-01-92$ & 246 & 8.0 & .05 & 1.74 & 1.79 & yes \\
\hline 44 & Winooski River & 2 & $7-01-92$ & 246 & 8.0 & .03 & .68 & .71 & yes \\
\hline 45 & Lake Bed near Winooski River & 1 & $6-23-92$ & -- & -- & .03 & 3.45 & 3.48 & yes \\
\hline 46 & Winooski River & 1 & $7-06-92$ & 236 & 8.0 & .04 & .82 & .86 & yes \\
\hline 46 & Winooski River & 1 & $7-06-92$ & 236 & 8.0 & .04 & .82 & .86 & no \\
\hline 49 & Highland Forge outlet & 1 & $7-27-92$ & 166 & 7.4 & .07 & .16 & .23 & no \\
\hline 50 & Lake Bed in shelburne Bay & 1 & $6-22-92$ & -- & -- & .05 & 3.49 & 3.54 & no \\
\hline 52 & Laplatte River & 1 & $7-07-92$ & 446 & 7.7 & .14 & .68 & .82 & yes \\
\hline 53 & Bouquet River & 1 & $7-27-92$ & 149 & 8.1 & .02 & .13 & .15 & no \\
\hline 53 & Bouquet River & 2 & $7-27-92$ & 149 & 8.1 & .03 & .99 & 1.02 & no \\
\hline 54 & Lake Bed near Bouquet River & 1 & $6-22-92$ & -- & -- & .03 & 1.73 & 1.76 & no \\
\hline 57 & Lewis Creek & 1 & $7-07-92$ & 248 & 8.8 & .19 & .43 & .62 & no \\
\hline 58 & Lake Bed near otter Creek & 1 & $6-22-92$ & -- & -- & .06 & 2.26 & 2.32 & yes \\
\hline 59 & Little Otter Creek & 1 & $6-15-92$ & 346 & 8.7 & -- & -- & -- & no \\
\hline 59 & Little Otter Creek & 2 & $6-15-92$ & 346 & 8.7 & -- & -- & -- & no \\
\hline 60 & Lake Bed near Hoisington River & $r \quad 1$ & $6-22-92$ & -- & -- & $<.01$ & 2.62 & 2.62 & yes \\
\hline 61 & Hoisington Brook & 1 & $7-10-92$ & 223 & 7.5 & .06 & .87 & .93 & no \\
\hline 62 & Otter Creek & 1 & $7-08-92$ & 230 & 8.5 & .03 & .70 & .73 & yes \\
\hline 67 & Mill Brook & 1 & $7-09-92$ & 149 & 8.2 & .09 & .70 & .79 & no \\
\hline 73 & Putnam Creek & 1 & $7-09-92$ & 297 & 8.3 & .60 & .82 & 1.42 & no \\
\hline 74 & Lake Bed near Putnam Creek & 1 & $6-30-92$ & -- & -- & .07 & 2.51 & 2.58 & no \\
\hline 74 & Lake Bed near Putnam Creek & 2 & $6-30-92$ & -- & -- & .07 & 2.50 & 2.57 & yes \\
\hline 74 & Lake Bed near Putnam Creek & 2 & $6-30-92$ & -- & -- & .07 & 2.50 & 2.57 & yes \\
\hline 78 & Ticonderoga Creek & 1 & $7-24-92$ & 124 & 8.0 & .34 & 8.01 & 8.35 & yes \\
\hline 78 & Ticonderoga Creek & 2 & $7-24-92$ & 124 & 8.0 & .34 & 8.78 & 9.12 & no \\
\hline 79 & Lake Bed near Lachutte & 1 & $6-30-92$ & -- & -- & .05 & 2.93 & 2.98 & yes \\
\hline 79 & Lake Bed near Lachutte & 1 & $6-30-92$ & -- & -- & .05 & 2.93 & 2.98 & yes \\
\hline 79 & Lake Bed near Lachutte & 2 & $6-30-92$ & -- & -- & .05 & 3.15 & 3.20 & yes \\
\hline 80 & East Creek & 1 & $7-16-92$ & 513 & 7.7 & .96 & 1.41 & 2.37 & no \\
\hline
\end{tabular}


Table 9. Carbonate, organic and total carbon concentrations, and polychlorinated biphenyl compound detections in streambed sediment for tributarles to Lake Champlain--Continued

\begin{tabular}{|c|c|c|c|c|c|c|c|c|c|}
\hline $\begin{array}{l}\text { Site } \\
\text { num- } \\
\text { ber' }\end{array}$ & Stream name & $\begin{array}{l}\text { Design } \\
\text { re- } \\
\text { marks² }\end{array}$ & Date & $\begin{array}{l}\text { Specific } \\
\text { conduct- } \\
\text { ance } \\
\text { (micro- } \\
\text { siemens } \\
\text { per centi- } \\
\text { meter) }\end{array}$ & $\begin{array}{l}\text { pH } \\
\text { (stand- } \\
\text { ard } \\
\text { units }\end{array}$ & $\begin{array}{l}\text { Carbon- } \\
\text { ate } \\
\text { carbon } \\
\text { (per- } \\
\text { cent) }\end{array}$ & $\begin{array}{l}\text { Organic } \\
\text { carbon } \\
\text { (per } \\
\text { cent) }\end{array}$ & $\begin{array}{l}\text { Total } \\
\text { carbon } \\
\text { (per- } \\
\text { cent) }\end{array}$ & $\begin{array}{l}\text { PCB's } \\
\text { de- } \\
\text { tected }\end{array}$ \\
\hline 83 & Mill Brook & 1 & $7-09-92$ & 311 & 8.0 & 0.54 & 0.86 & 1.40 & no \\
\hline 89 & Poultney River & 1 & $7-16-92$ & 215 & 7.7 & .31 & .19 & .50 & no \\
\hline 89 & Poultney River & 2 & $7-16-92$ & 215 & 7.7 & .20 & .11 & .31 & no \\
\hline 95 & Metawee River & 1 & $7-15-92$ & 230 & 7.9 & .05 & 1.79 & 1.84 & no \\
\hline 97 & Mount Hope Brook & 1 & $7-17-92$ & 86 & 8.2 & .13 & .25 & .38 & no \\
\hline
\end{tabular}

${ }^{1}$ Location of site number shown in figure 1.

${ }^{2}$ Remark code 1 means a normal sample; 2 means same-day resampling of site; some samples were split at the laboratory and are indicated by a second sample with the same site number and remark code, either 1 or 2 .

${ }^{3}$ No means PCB's were not detected according to the criterion given in the methods section; yes means PCB's were detected according to the criterion given in the methods section. 


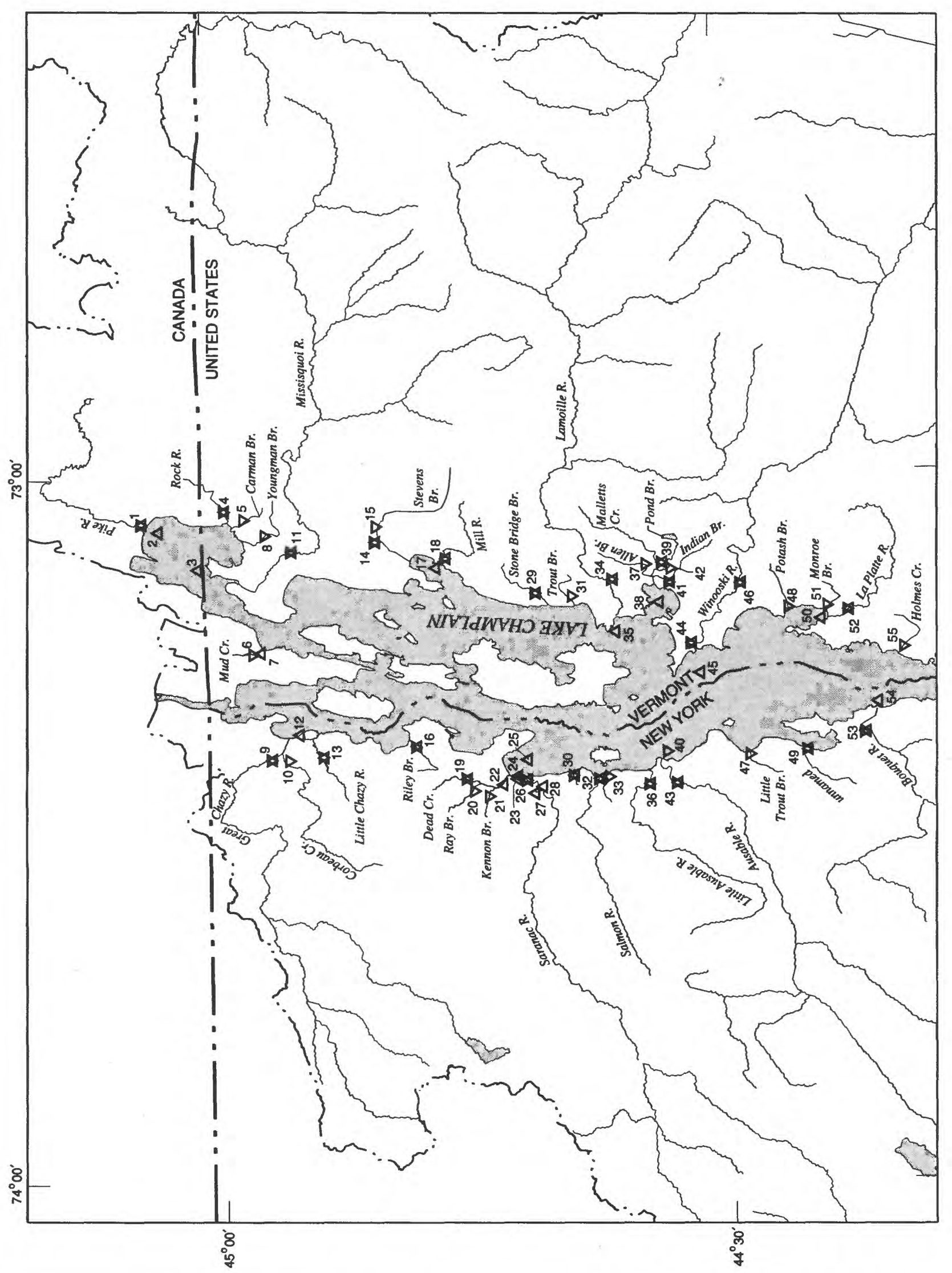




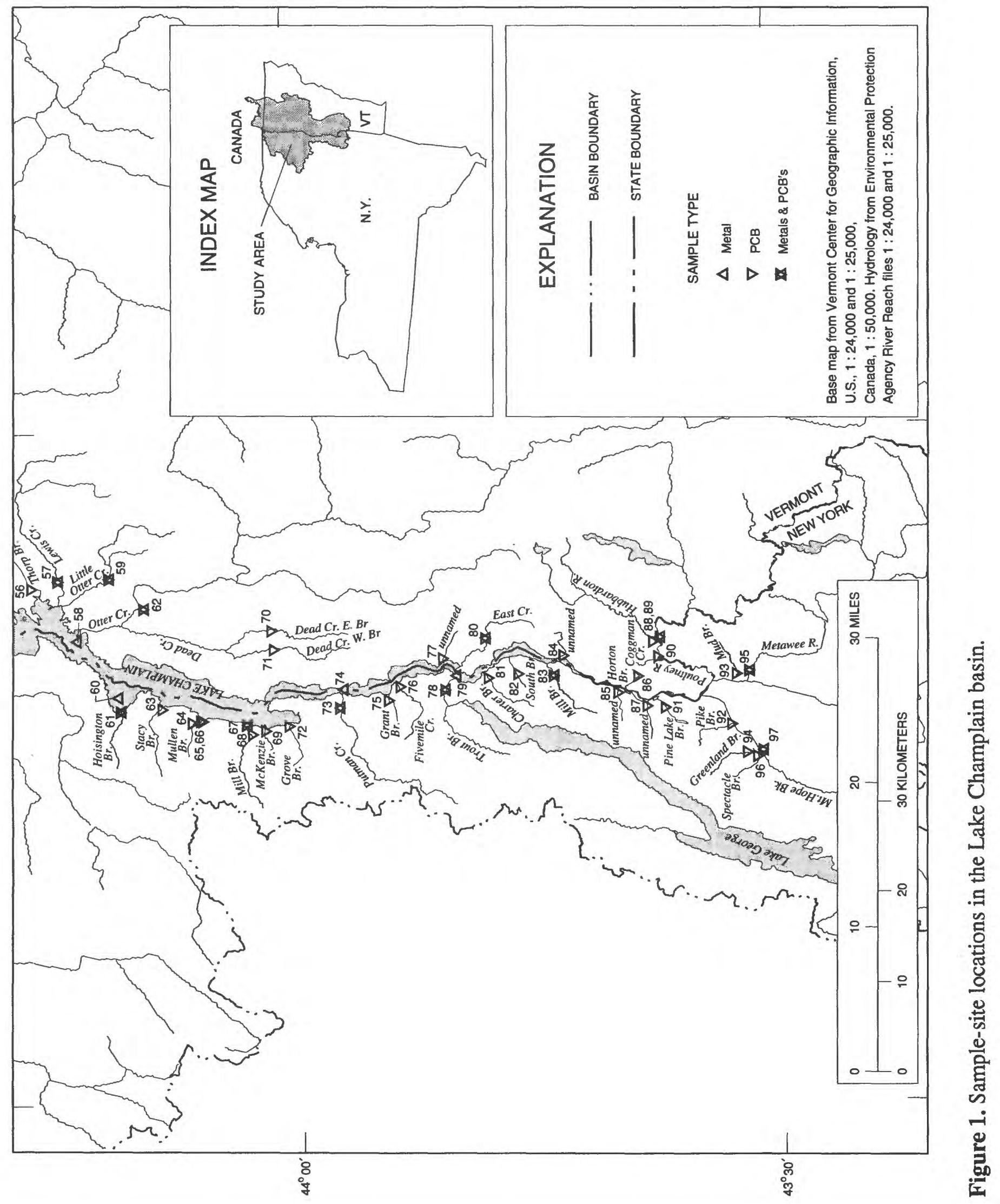

Flgure 149 


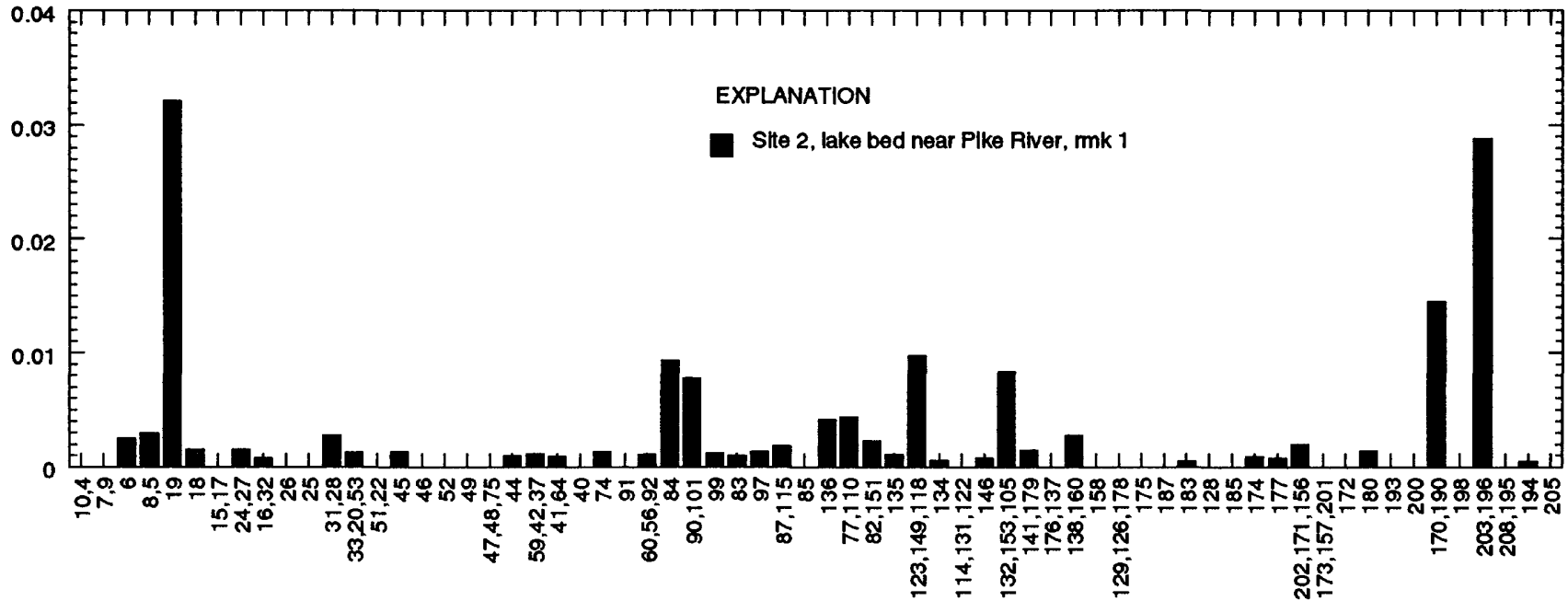

POLYCHLORINATED BIPHENYL CONGENER NUMBER

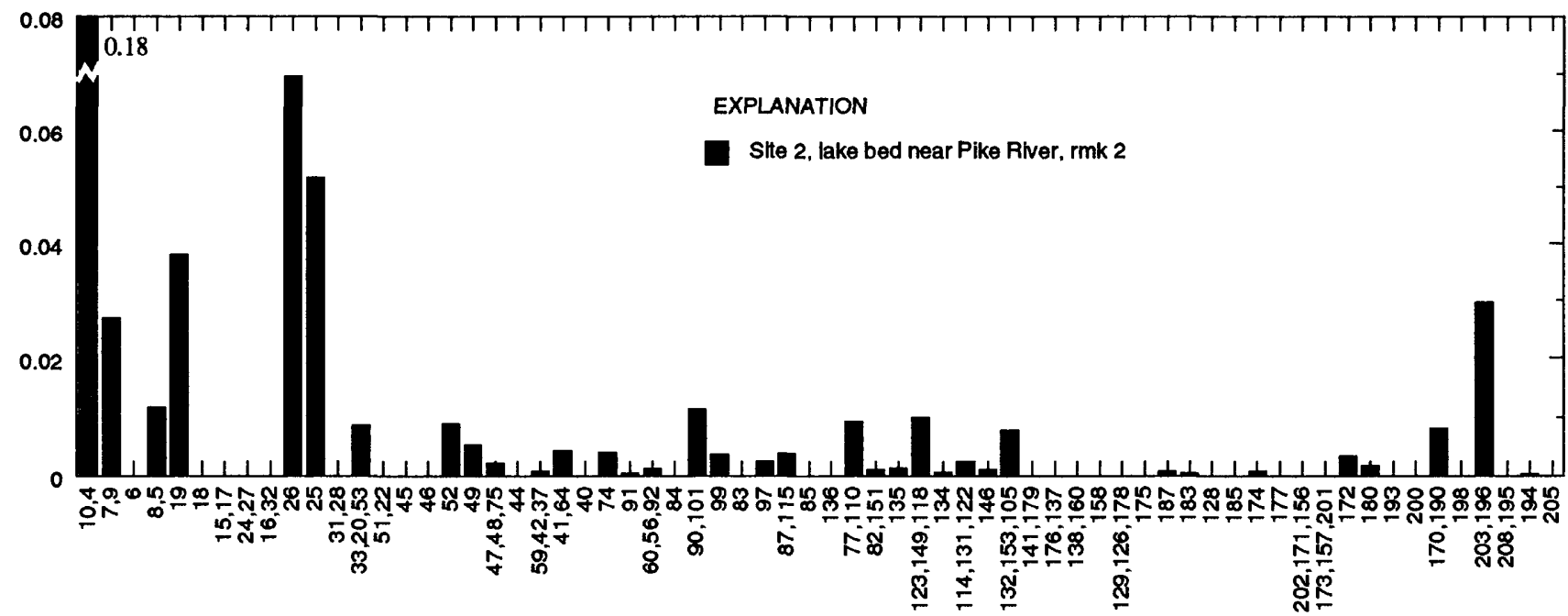

POLYCHLORINATED BIPHENYL CONGENER NUMBER

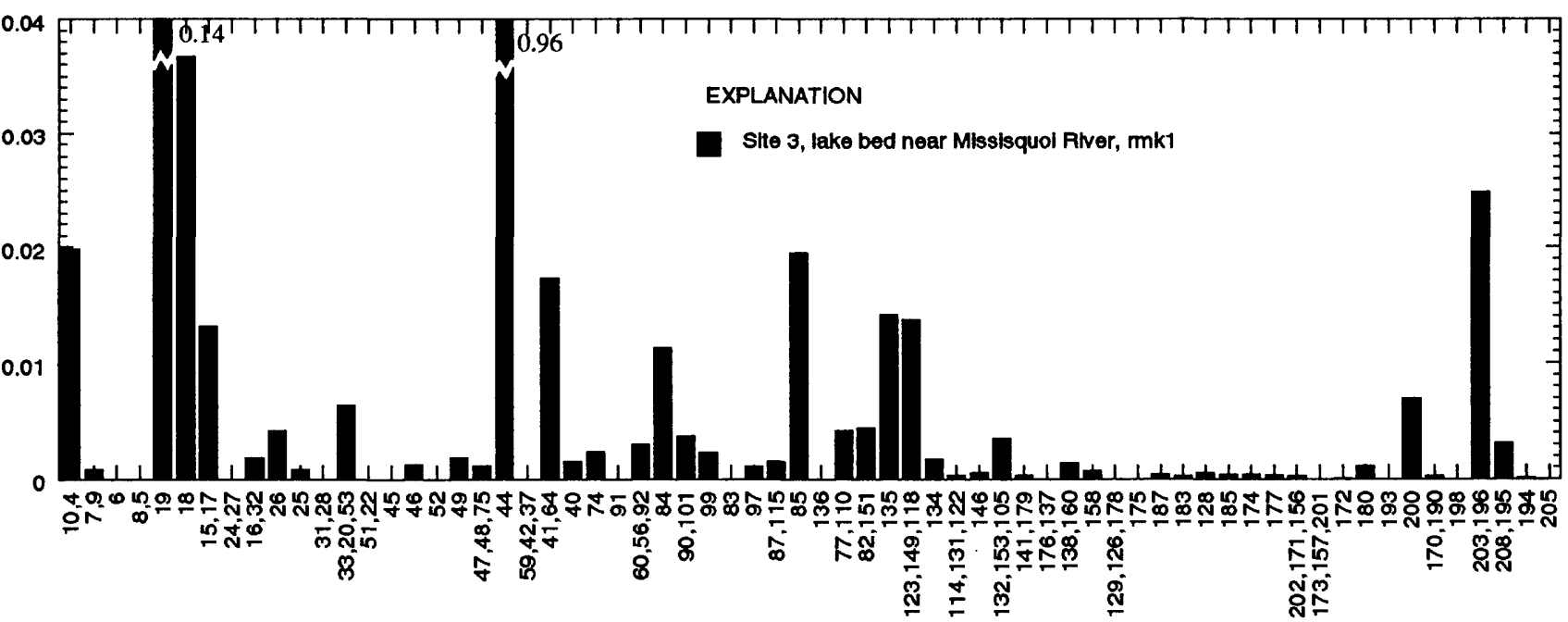

POLYCHLORINATED BIPHENYL CONGENER NUMBER

Figure 2. Histograms of polychlorinated biphenyl congeners (PCBs) from stream- and lake-bed samples of Lake Champlain. Site numbers are listed in figure 1 and site descriptions in table 1. Congener identifications are listed by International Union of Practical and Applied Chemistry number in table 7. Concentration units apply only if height of peak is caused by PCB congeners as described in text; rmk refers to Design remark in table 9.

50 Geochemical Data on Concentrations of Inorganic Constituents and Polychiorinated Biphenyi Congeners in Streambed Sediments in Tributaries to Lake Champialn In New York, Vermont, and Quebec, 1992 


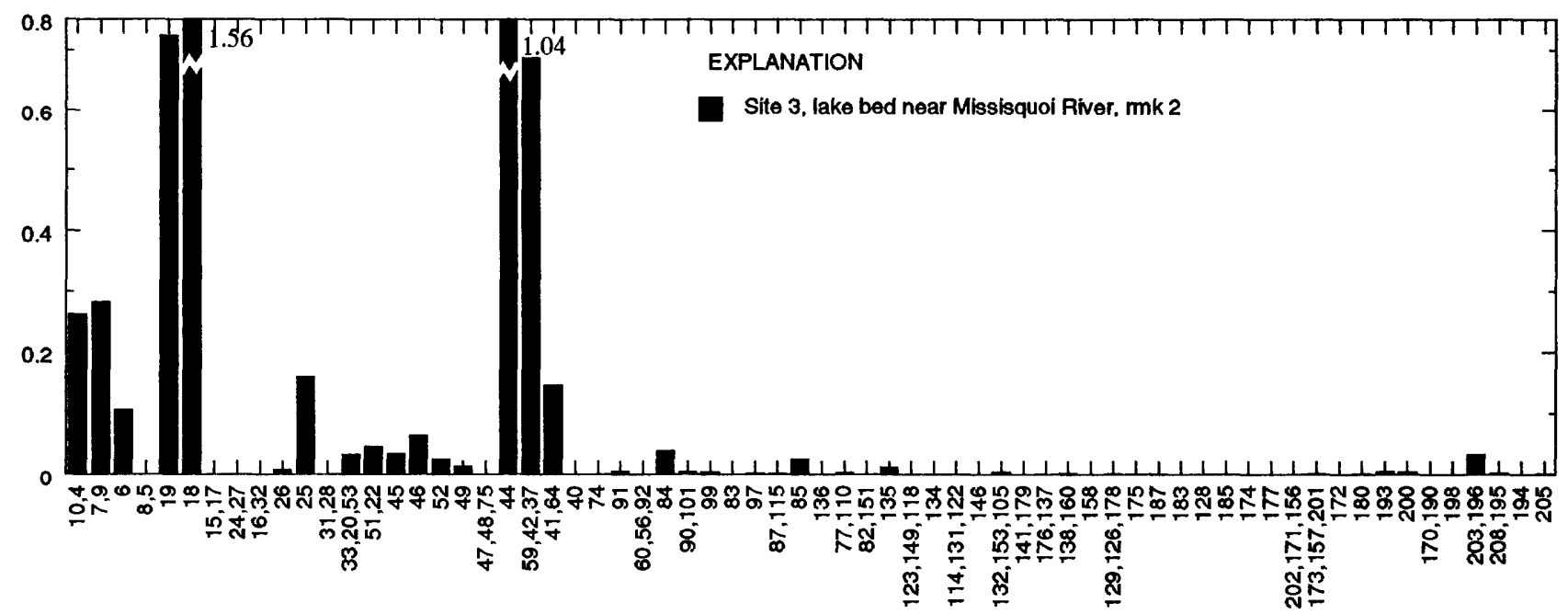

POLYCHLORINATED BIPHENYL CONGENER NUMBER

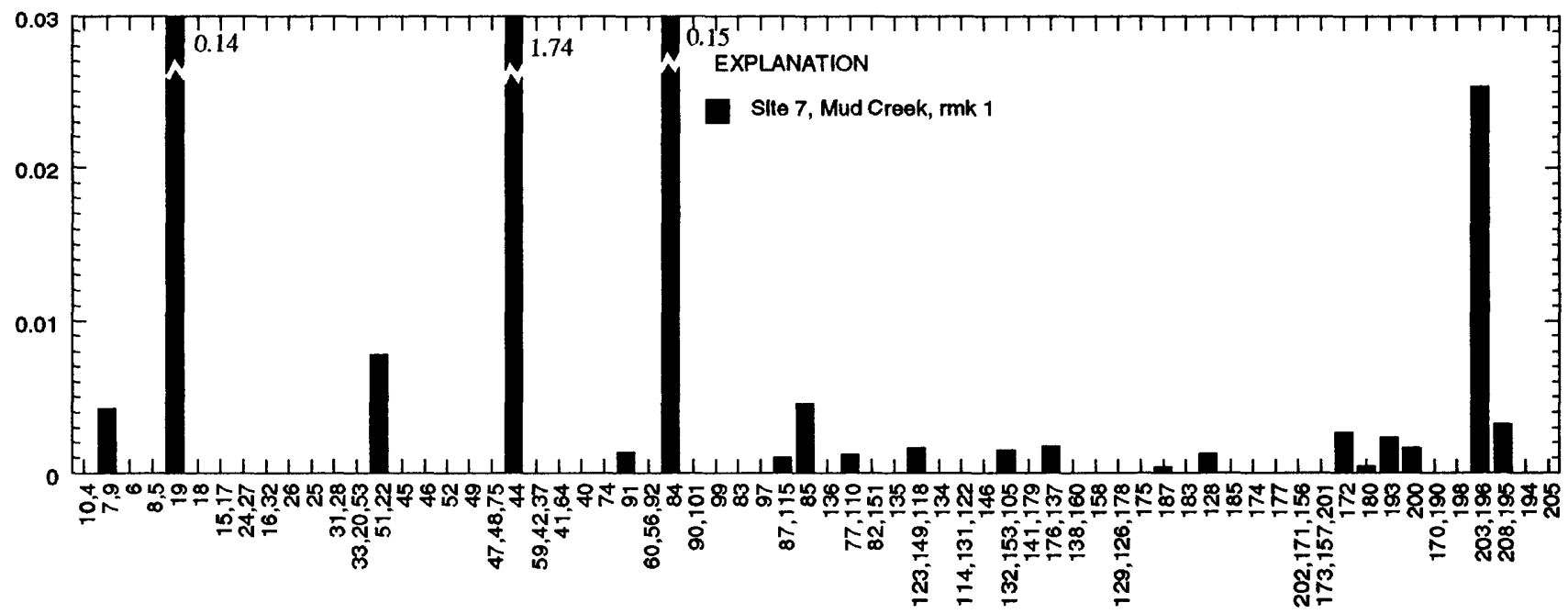

POLYCHLORINATED BIPHENYL CONGENER NUMBER

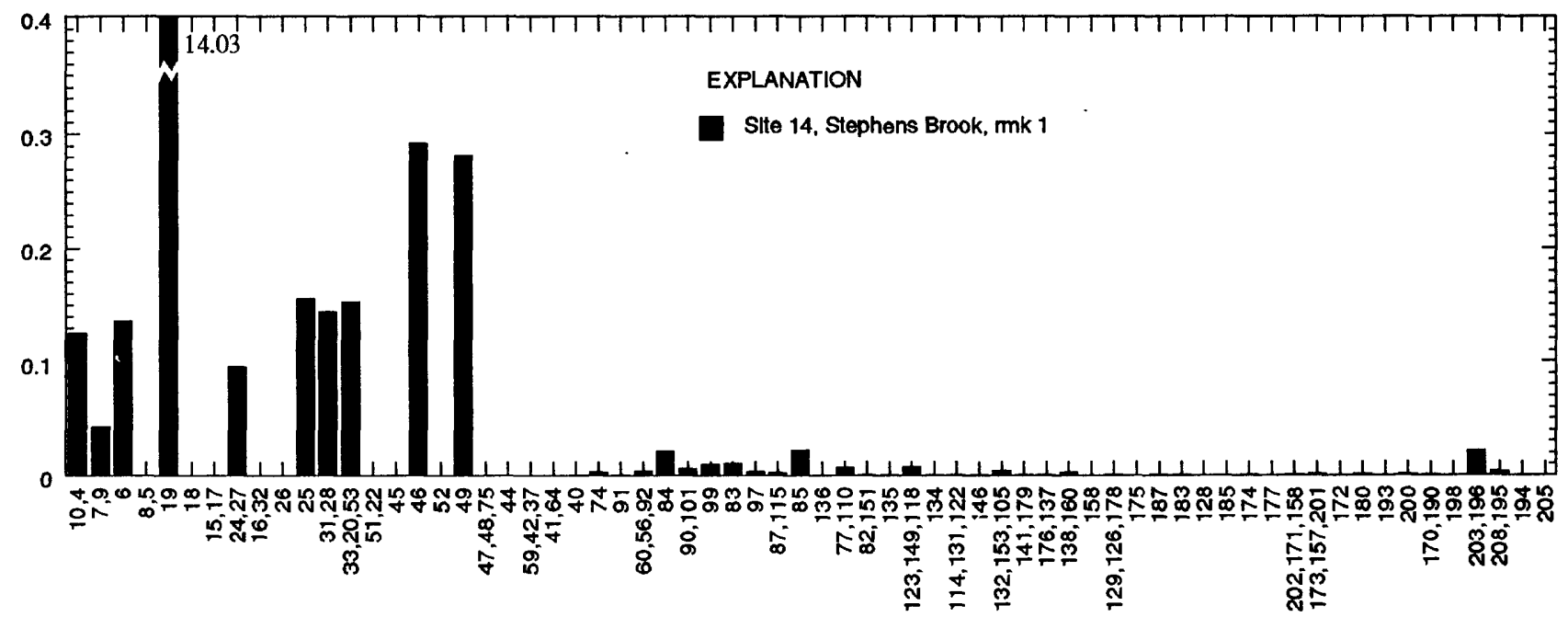

POLYCHLORINATED BIPHENYL CONGENER NUMBER

Figure 2. Histograms of polychlorinated biphenyl congeners (PCBs) from stream- and lake-bed samples of Lake Champlain. Site numbers are listed in figure 1 and site descriptions in table 1. Congener identifications are listed by International Union of Practical and Applied Chemistry number in table 7. Concentration units apply only if height of peak is caused by PCB congeners as described in text; rmk refers to Design remark in table 9.--Continued 


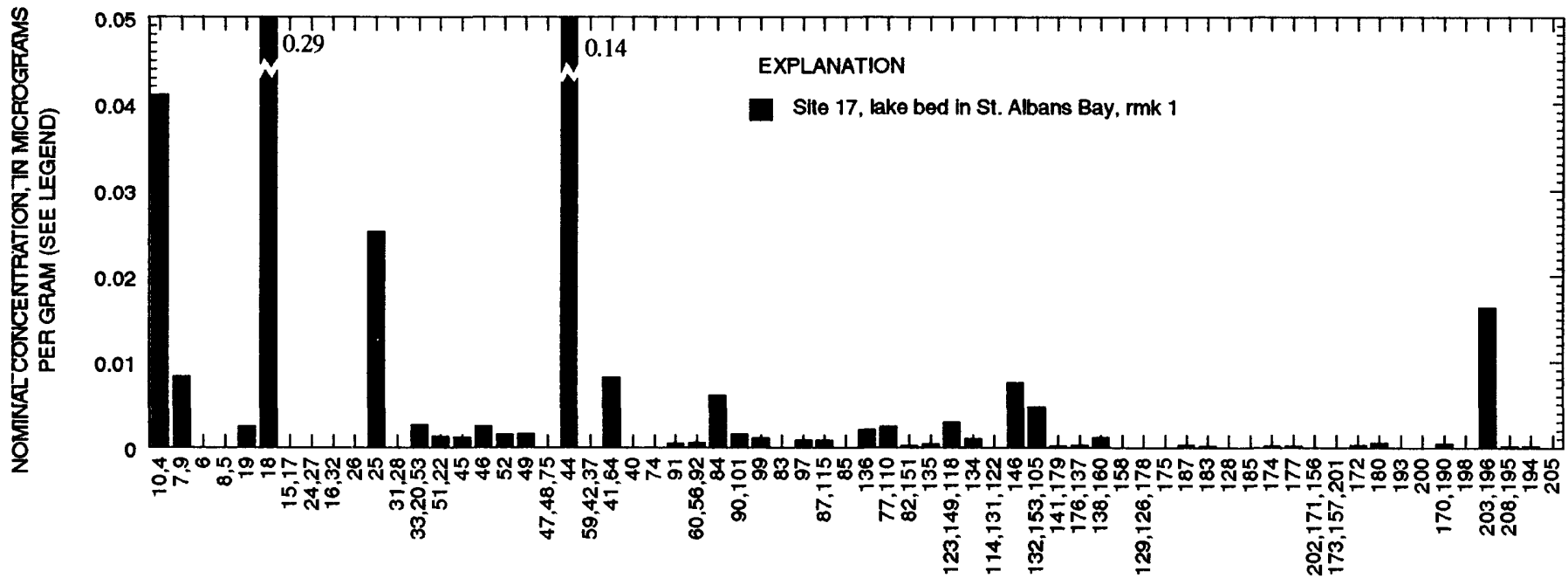

POLYCHLORINATED BIPHENYL CONGENER NUMBER

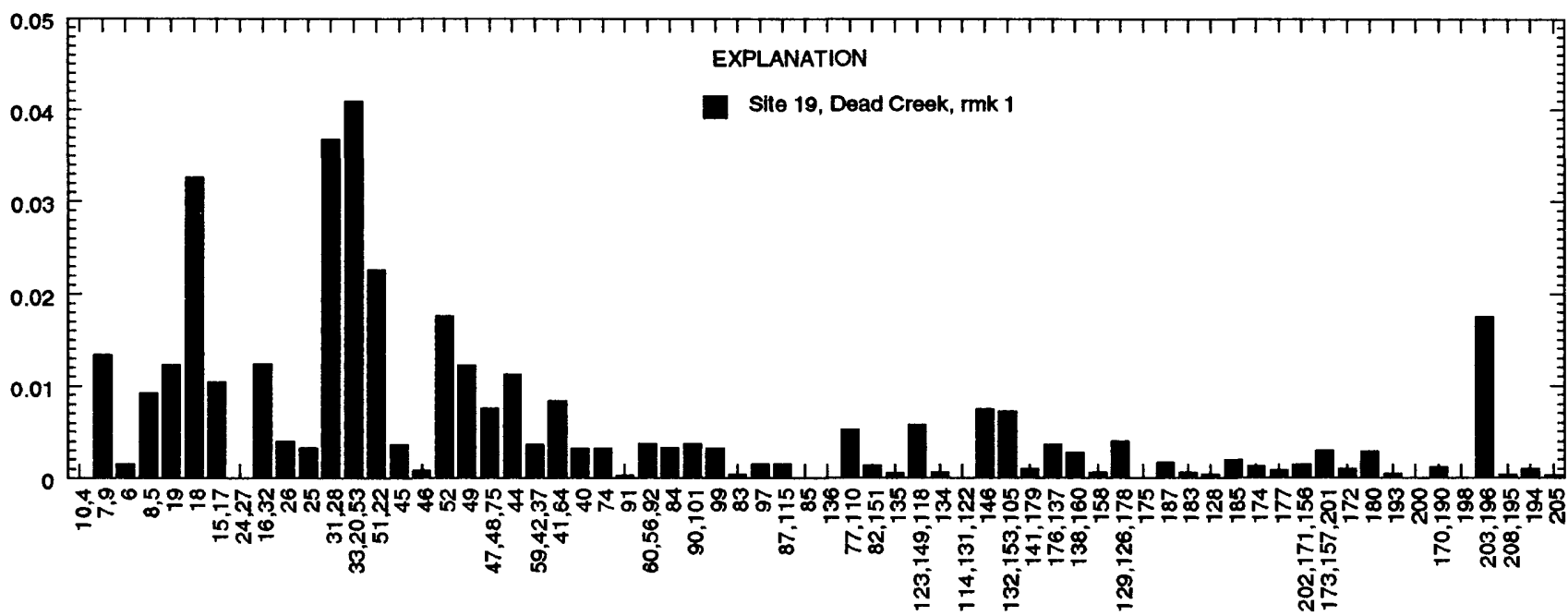

POLYCHLORINATED BIPHENYL CONGENER NUMBER
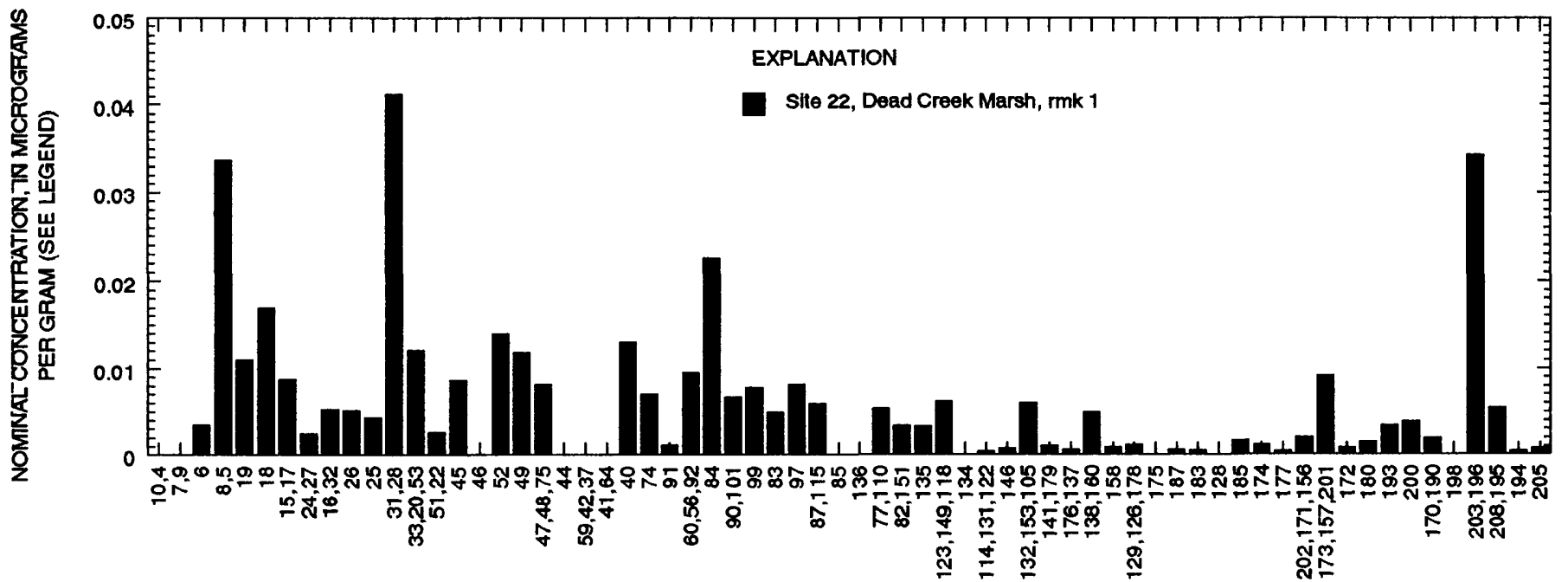

POLYCHLORINATED BIPHENYL CONGENER NUMBER

Figure 2. Histograms of polychlorinated biphenyl congeners (PCBs) from stream- and lake-bed samples of Lake Champlain. Site numbers are listed in figure 1 and site descriptions in table 1. Congener identifications are listed by International Union of Practical and Applied Chemistry number in table 7. Concentration units apply only if height of peak is caused by PCB congeners as described in text; rmk refers to Design remark in table 9.-.Continued 


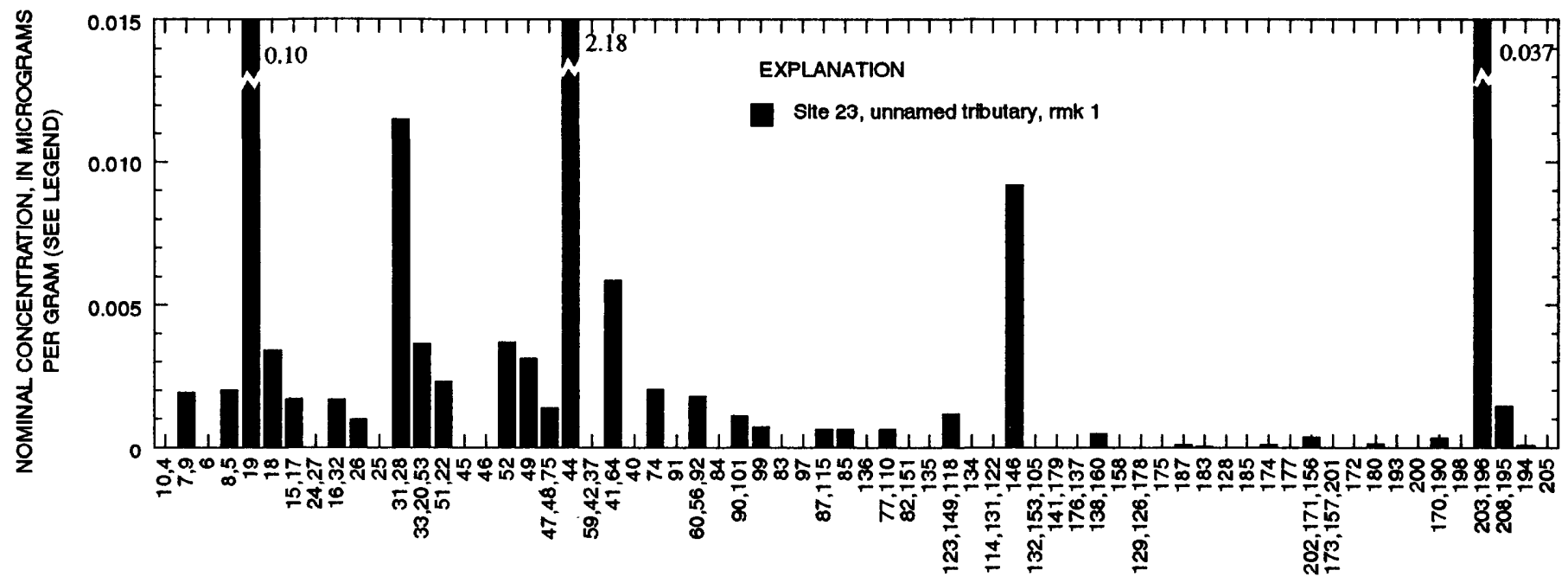

POLYCHLORINATED BIPHENYL CONGENER NUMBER

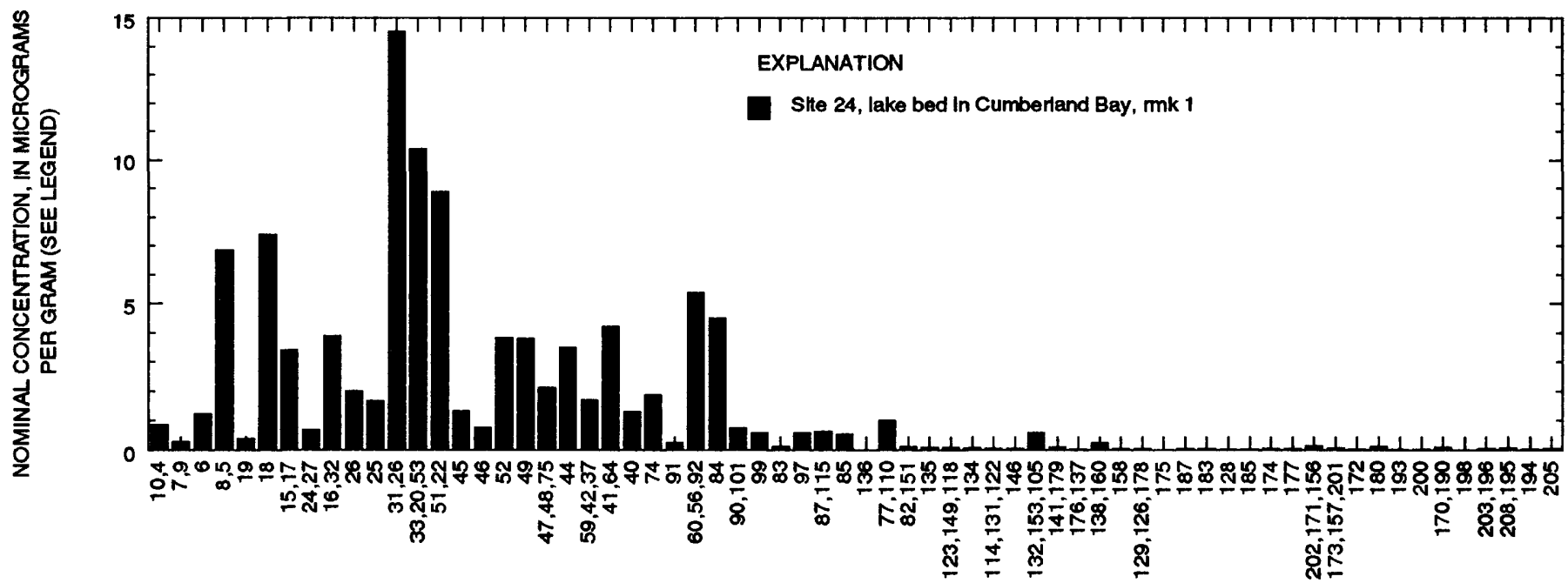

POLYCHLORINATED BIPHENYL CONGENER NUMBER

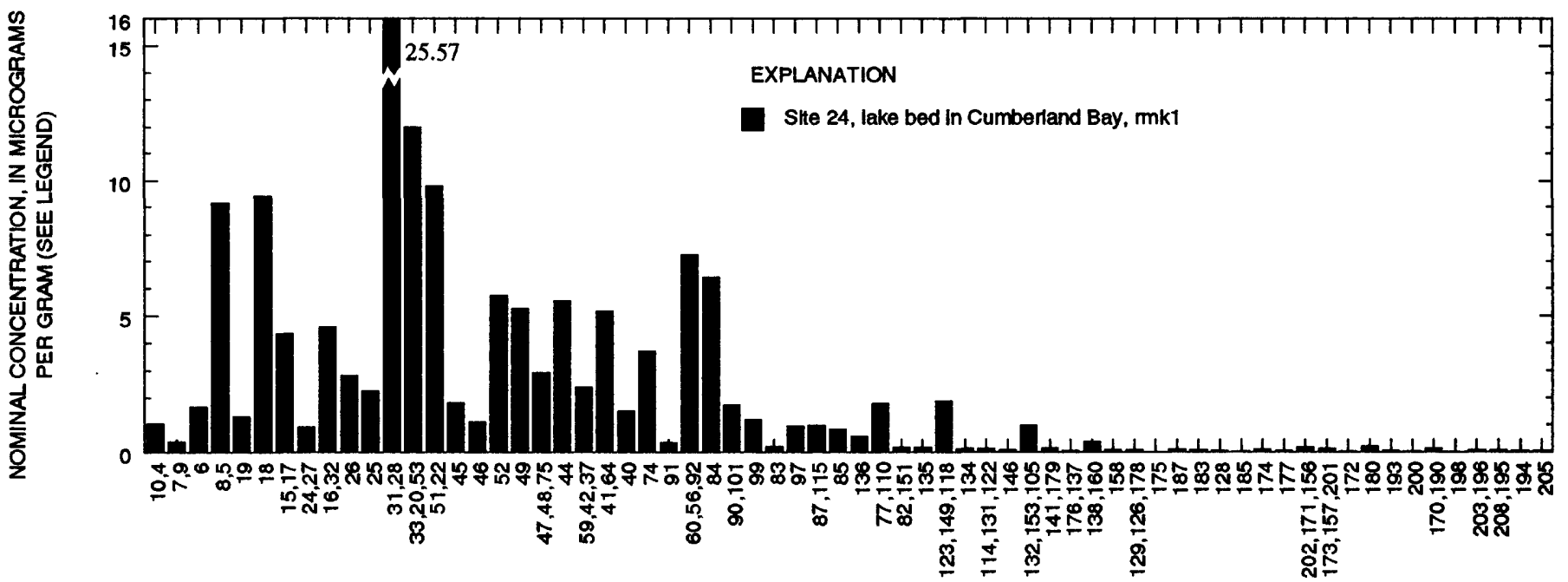

POLYCHLORINATED BIPHENYL CONGENER NUMBER

Figure 2. Histograms of polychlorinated biphenyl congeners (PCBs) from stream- and lake-bed samples of Lake Champlain. Site numbers are listed in figure 1 and site descriptions in table 1. Congener identifications are listed by International Union of Practical and Applied Chemistry number in table 7. Concentration units apply only if height of peak is caused by PCB congeners as described in text; rmk refers to Design remark in table 9.--Continued 


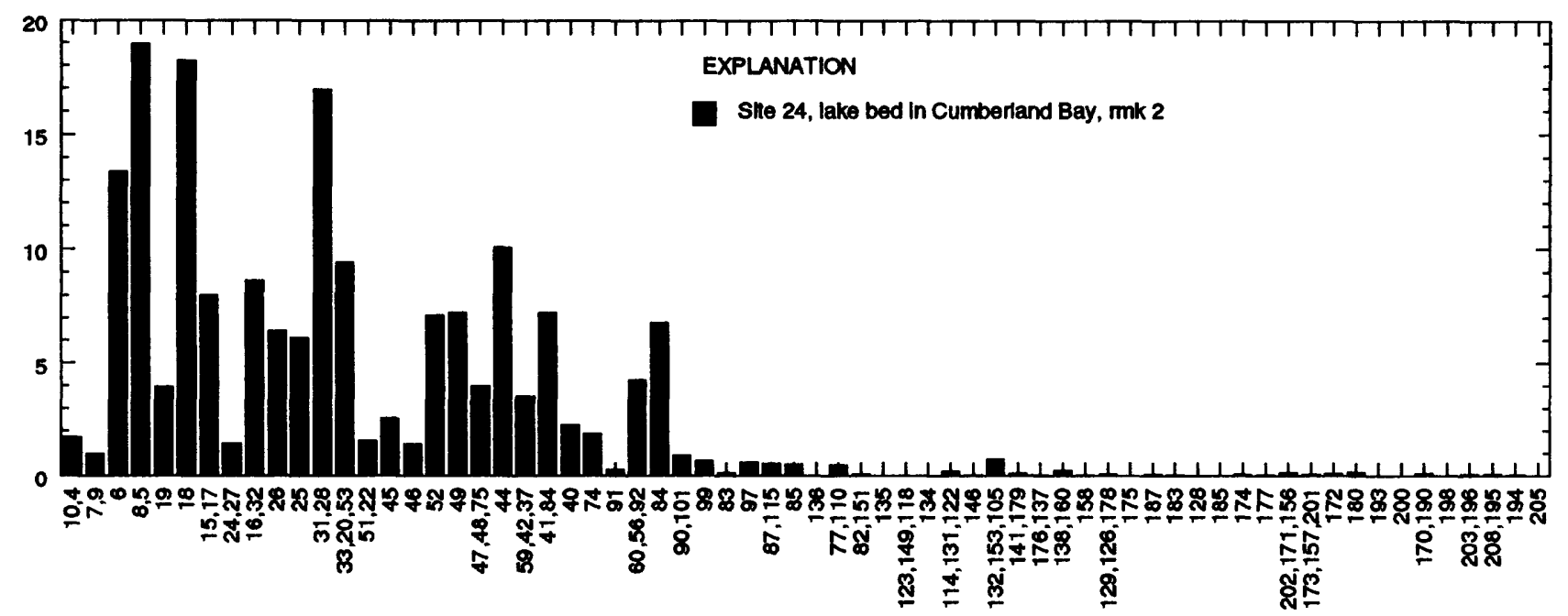

POLYCHLORINATED BIPHENYL CONGENER NUMBER

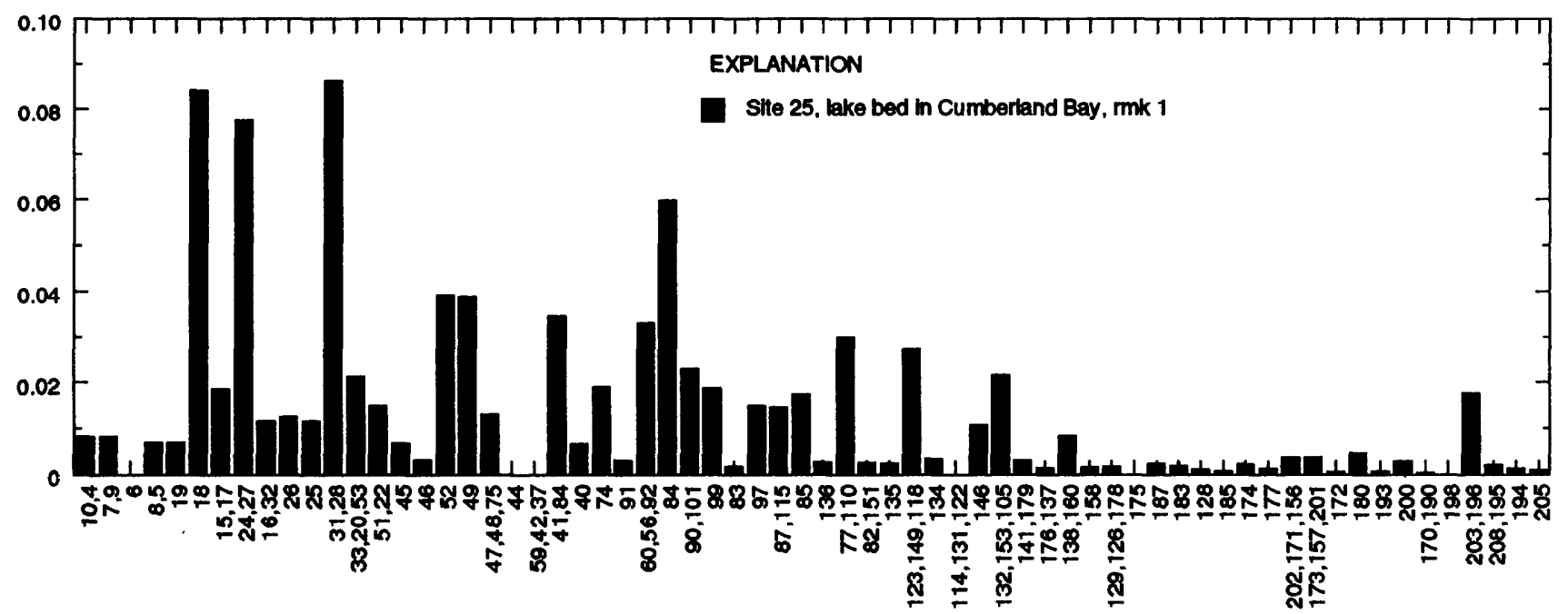

POLYCHLORINATED BIPHENYL CONGENER NUMBER

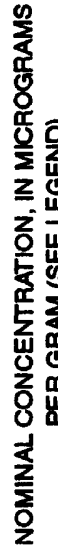

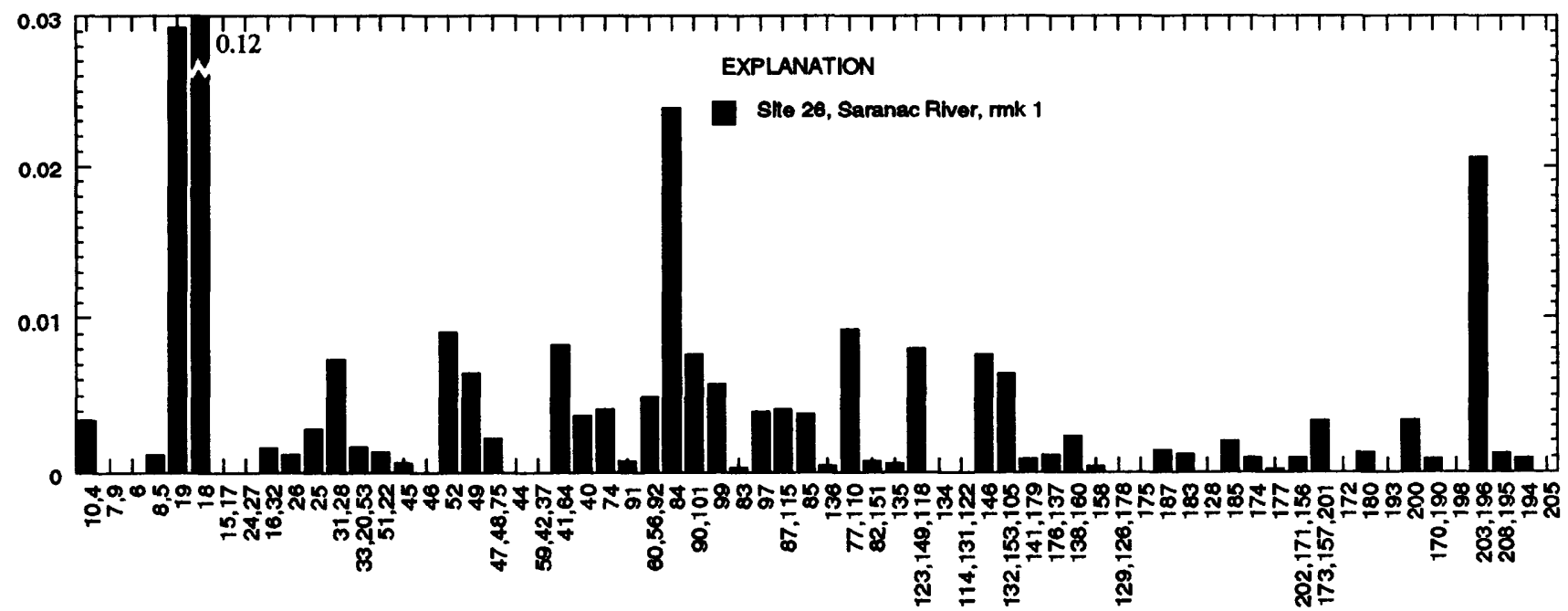

POLYCHLORINATED BIPHENYL CONGENER NUMBER

Figure 2. Histograms of polychlorinated biphenyl congeners (PCBs) from stream- and lake-bed samples of Lake Champlain. Site numbers are listed in figure 1 and site descriptions in table 1. Congener identifications are listed by International Union of Practical and Applied Chemistry number in table 7. Concentration units apply only if height of peak is caused by PCB congeners as described in text; rmk refers to Design remark in table 9.--Continued 


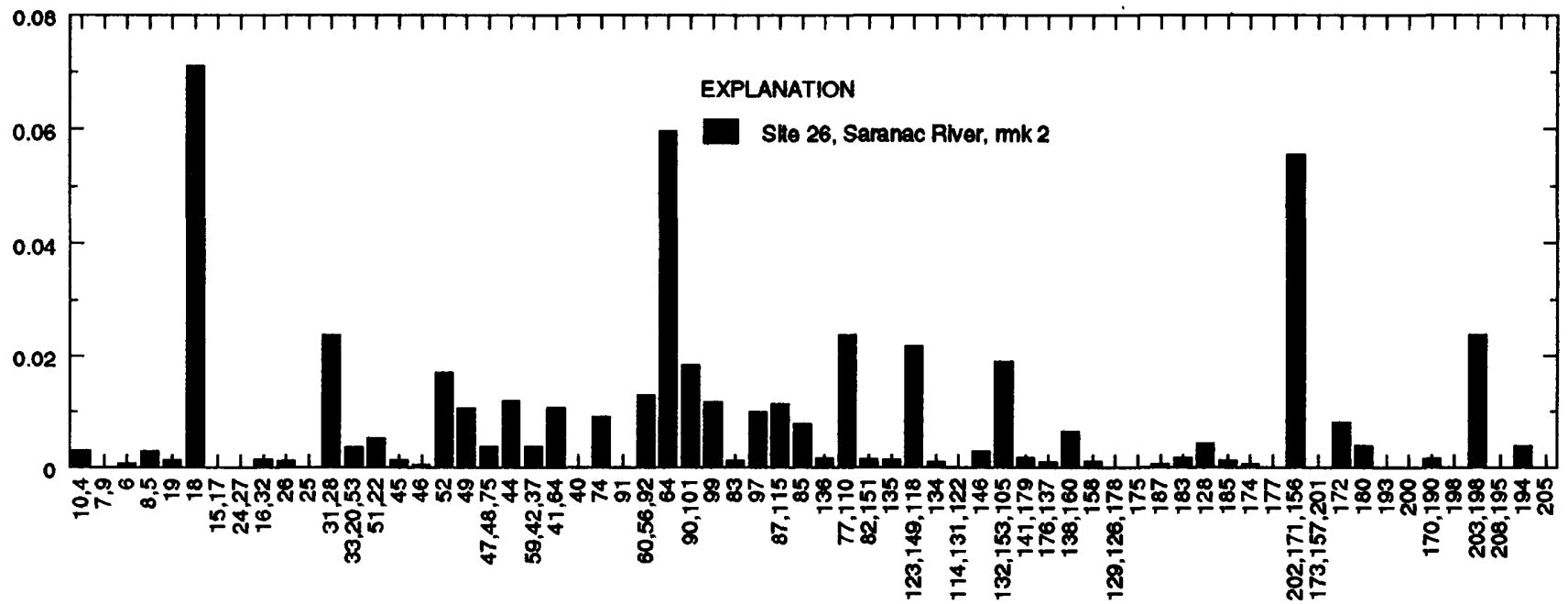

POLYCHLORINATED BIPHENYL CONGENER NUMBER

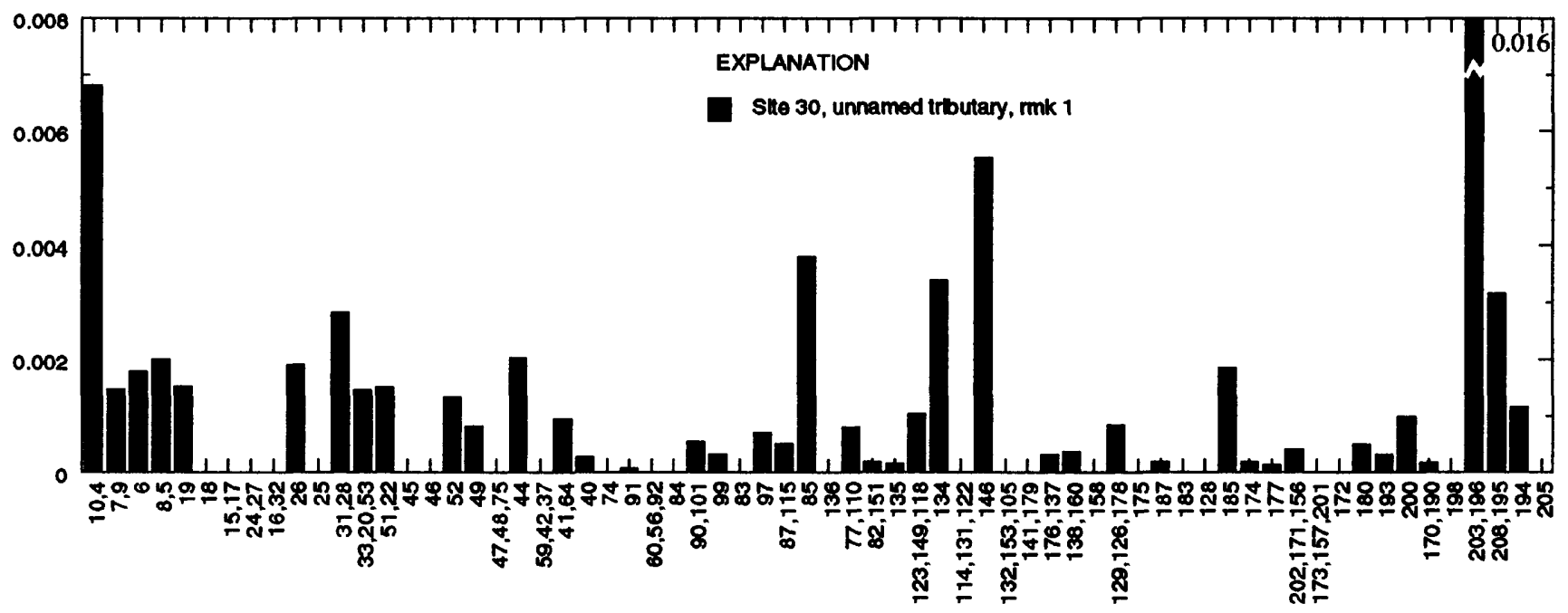

POLYCHLORINATED BIPHENYL CONGENER NUMBER
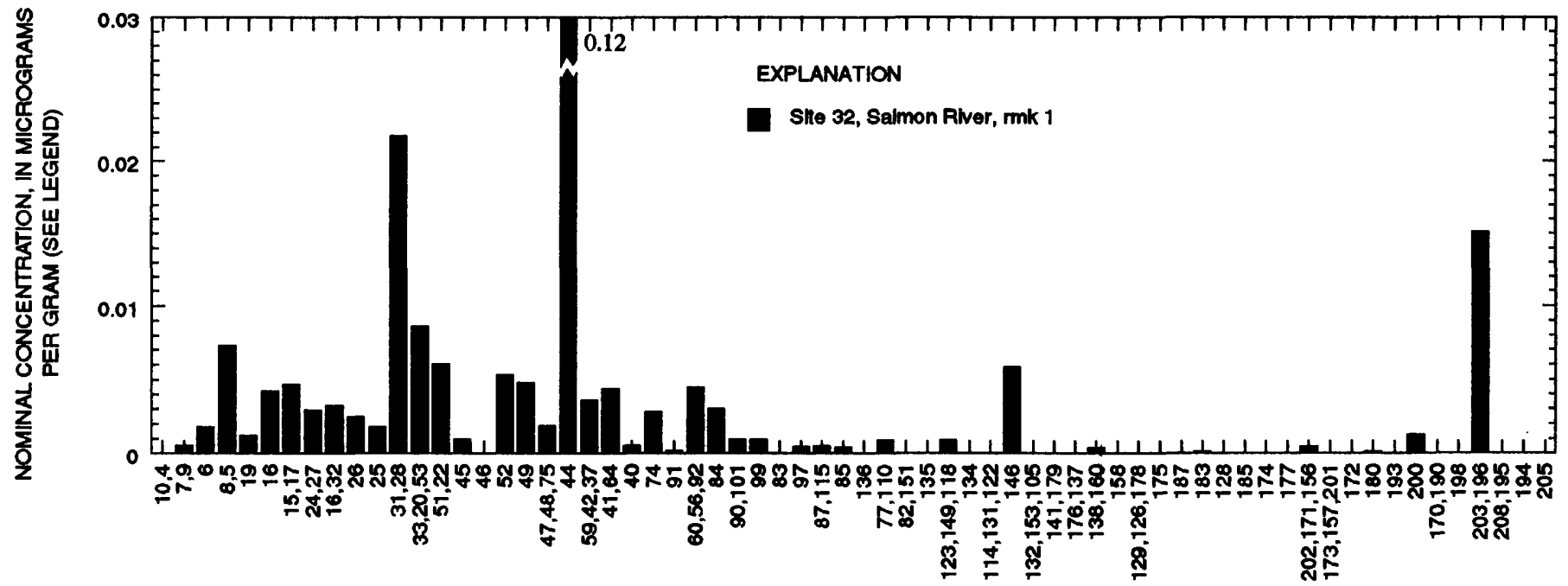

POLYCHLORINATED BIPHENYL CONGENER NUMBER

Figure 2. Histograms of polychlorinated biphenyl congeners (PCBs) from stream- and lake-bed samples of Lake Champlain. Site numbers are listed in figure 1 and site descriptions in table 1. Congener identifications are listed by International Union of Practical and Applied Chemistry number in table 7. Concentration units apply only if height of peak is caused by PCB congeners as described in text; rmk refers to Design remark in table 9.--Continued 


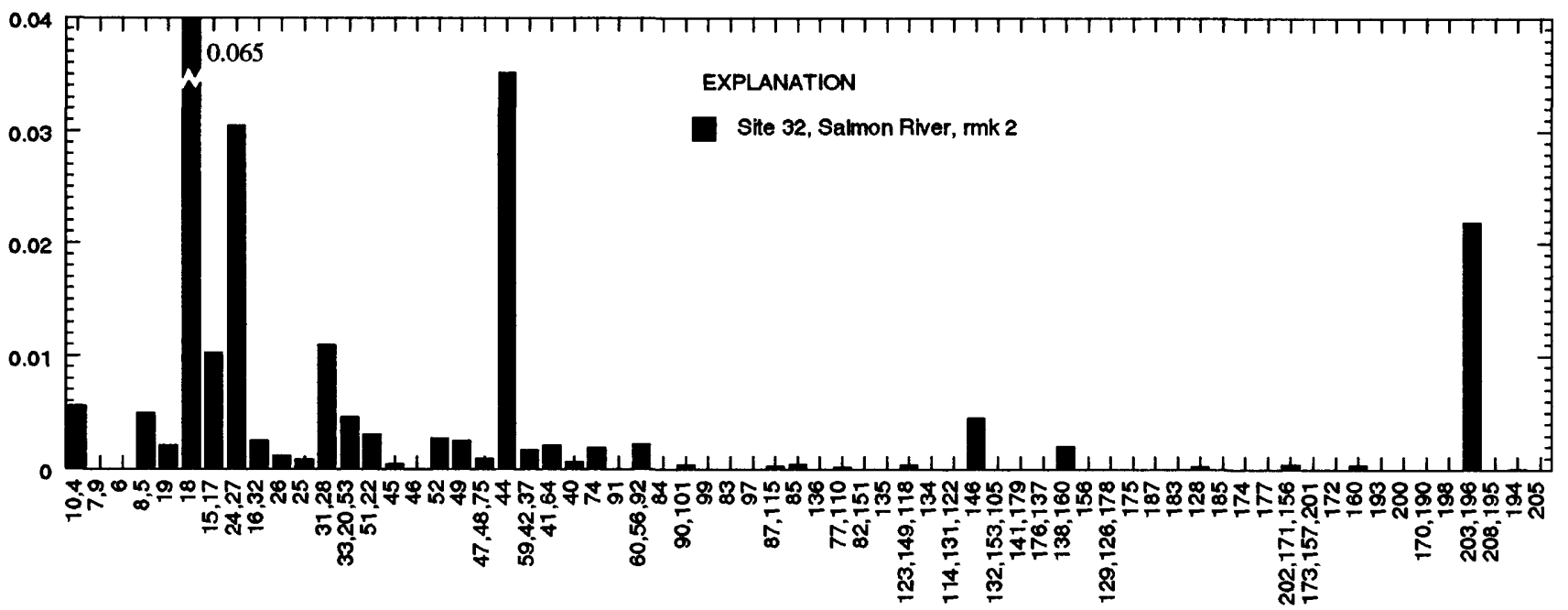

POLYCHLORINATED BIPHENYL CONGENER NUMBER

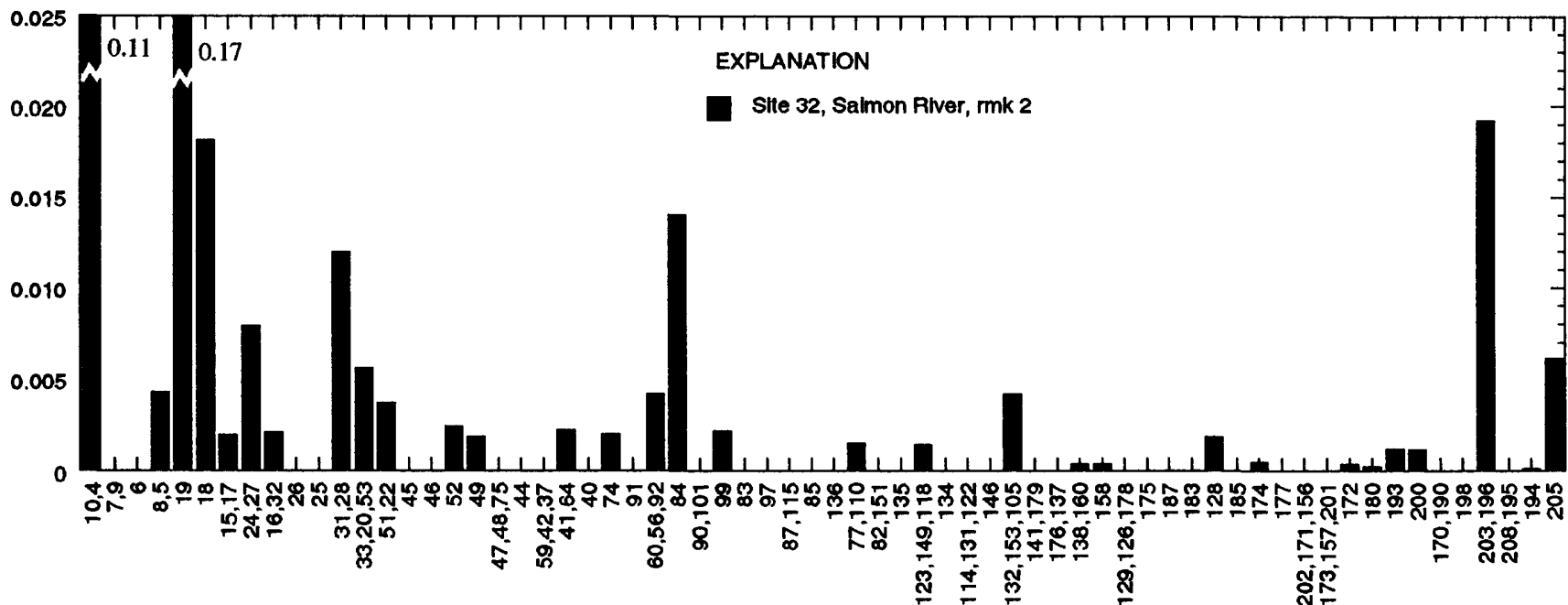

POLYCHLORINATED BIPHENYL CONGENER NUMBER

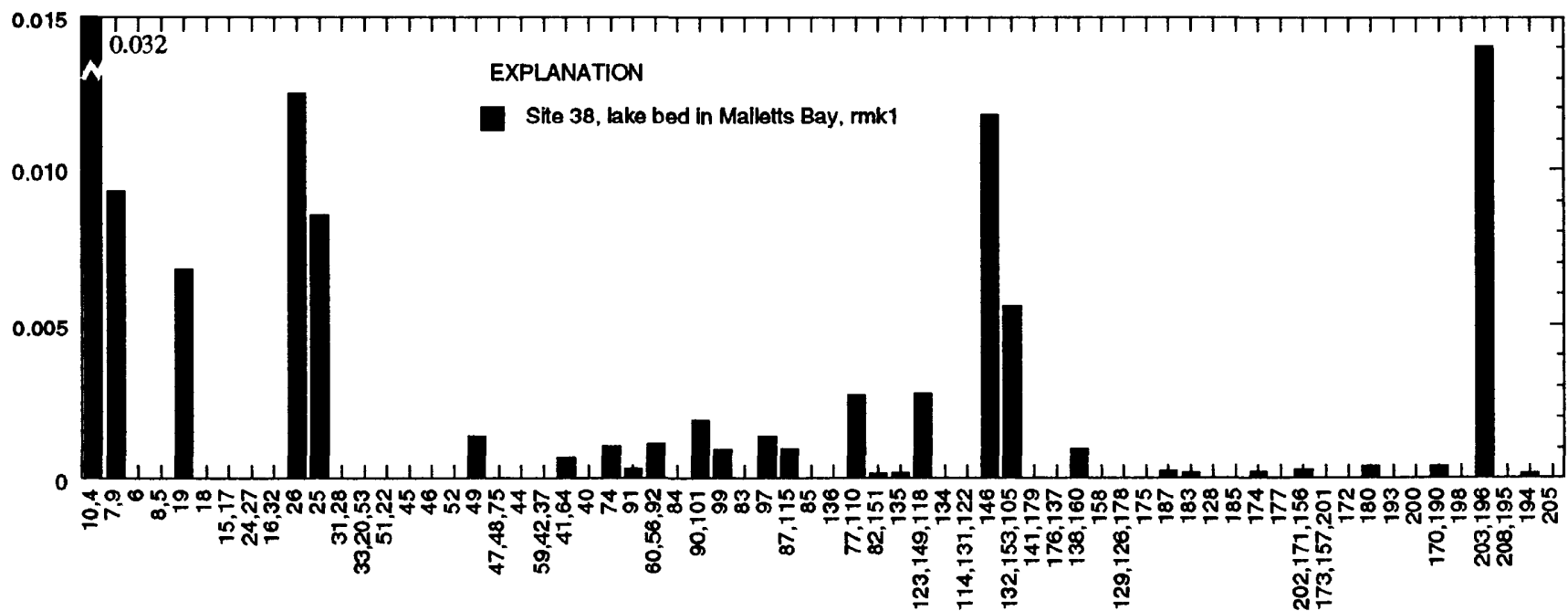

POLYCHLORINATED BIPHENYL CONGENER NUMBER

Figure 2. Histograms of polychlorinated biphenyl congeners (PCBs) from stream- and lake-bed samples of Lake Champlain. Site numbers are listed in figure 1 and site descriptions in table 1. Congener identifications are listed by International Union of Practical and Applied Chemistry number in table 7. Concentration units apply only if height of peak is caused by PCB congeners as described in text; rmk refers to Design remark in table 9.--Continued

56 Geochemical Data on Concentrations of Inorganic Constituents and Polychlorinated Blphenyl Congenere in Streambed 


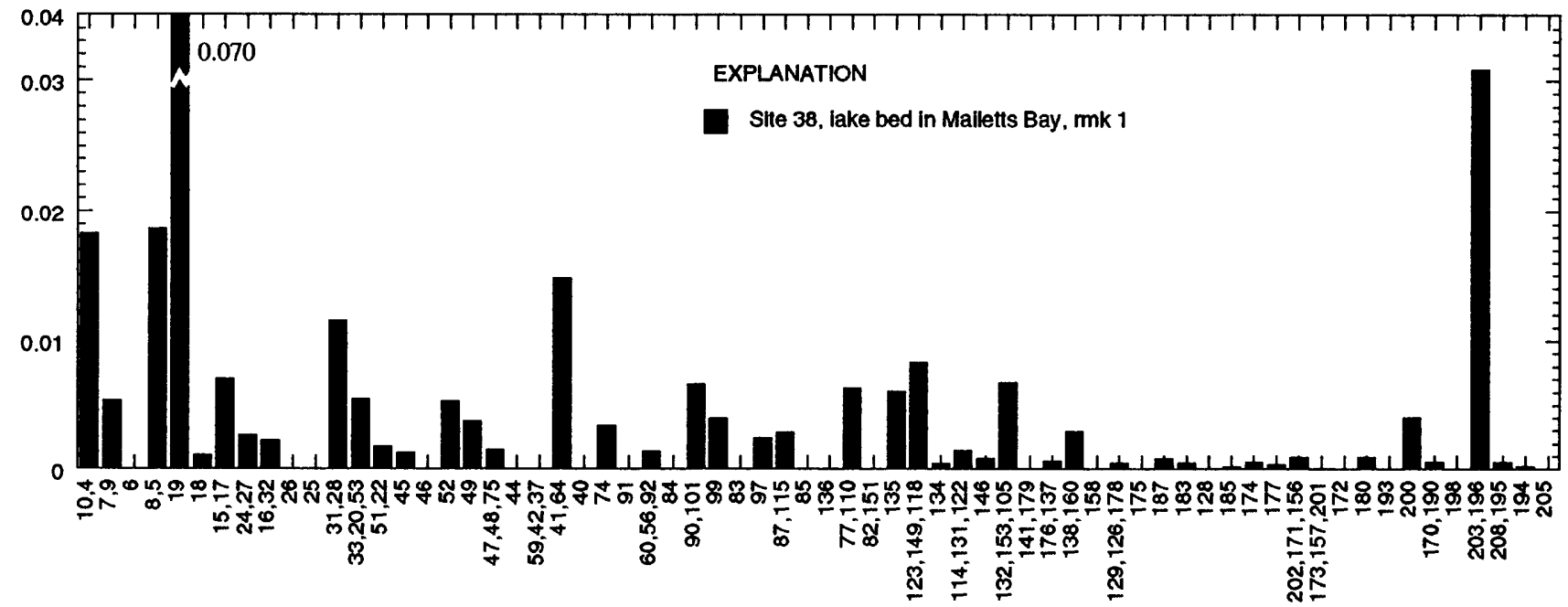

POLYCHLORINATED BIPHENYL CONGENER NUMBER

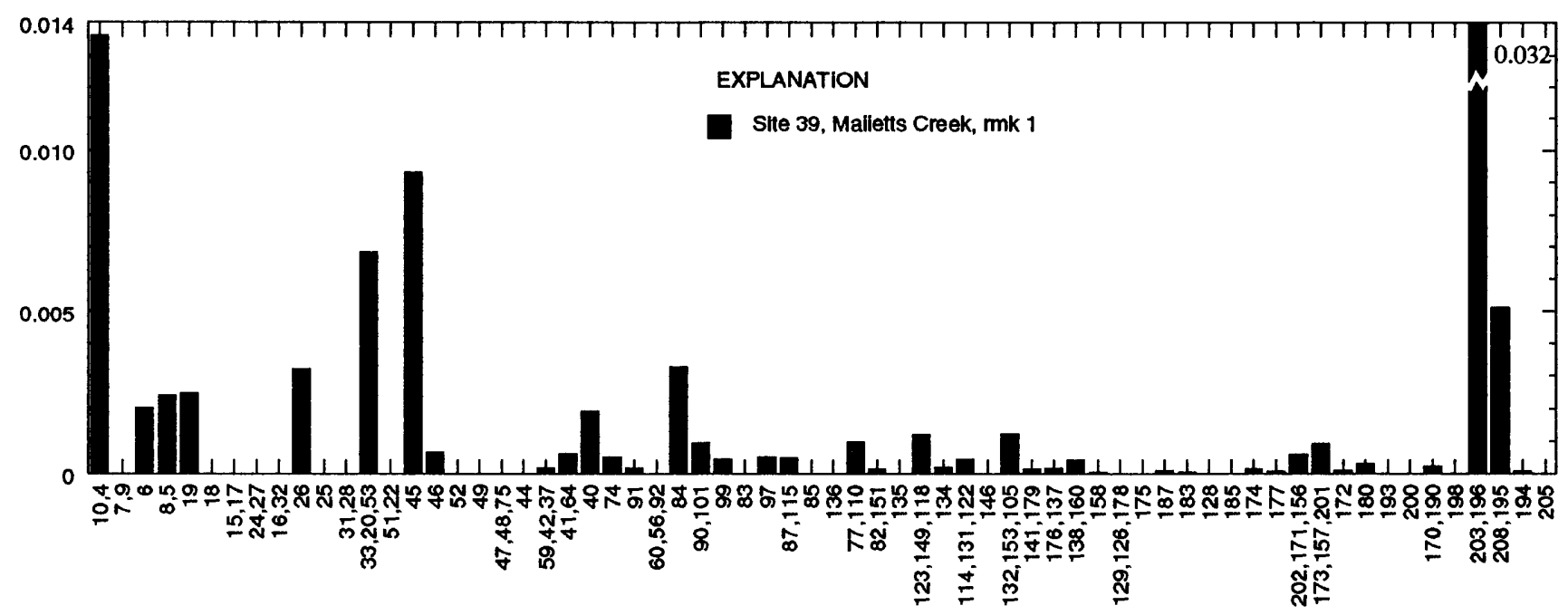

POLYCHLORINATED BIPHENYL CONGENER NUMBER

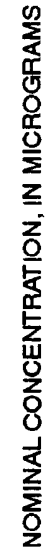

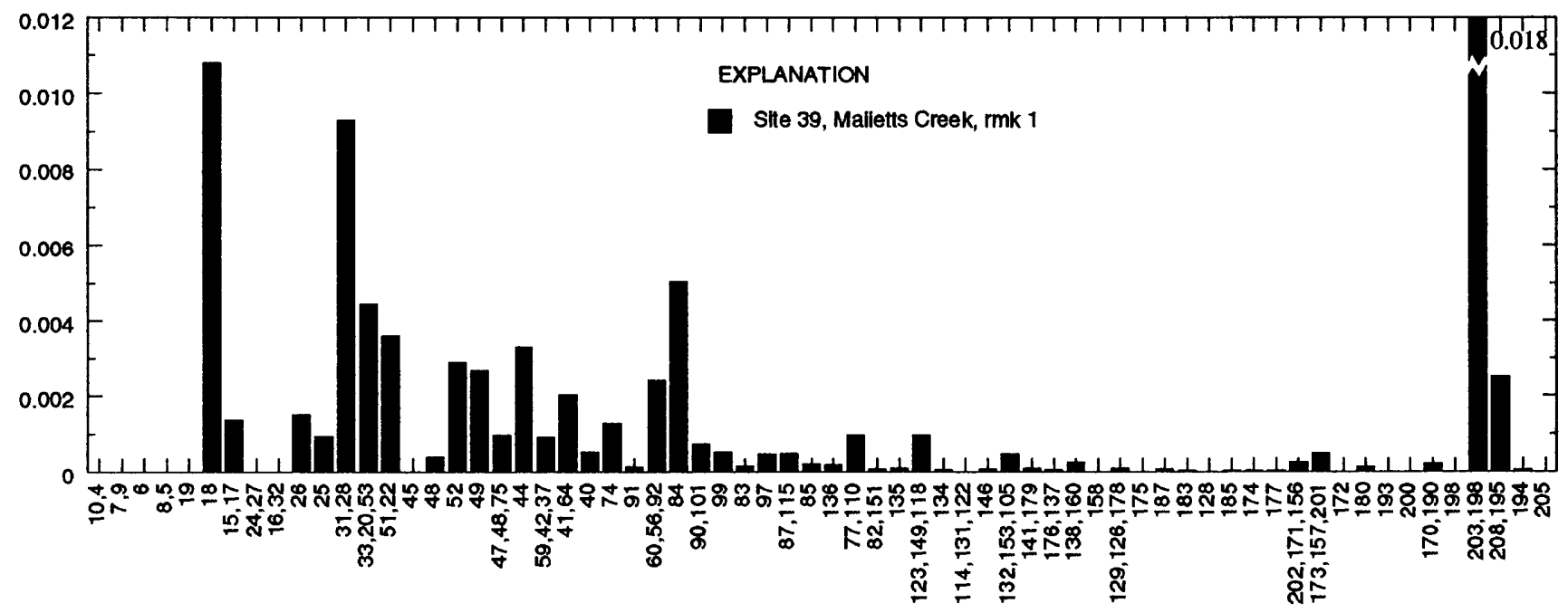

POLYCHLORINATED BIPHENYL CONGENER NUMBER

Figure 2. Histograms of polychlorinated biphenyl congeners (PCBs) from stream- and lake-bed samples of Lake Champlain. Site numbers are listed in figure 1 and site descriptions in table 1. Congener identifications are listed by International Union of Practical and Applied Chemistry number in table 7. Concentration units apply only if height of peak is caused by PCB congeners as described in text; rmk refers to Design remark in table 9.--Continued 


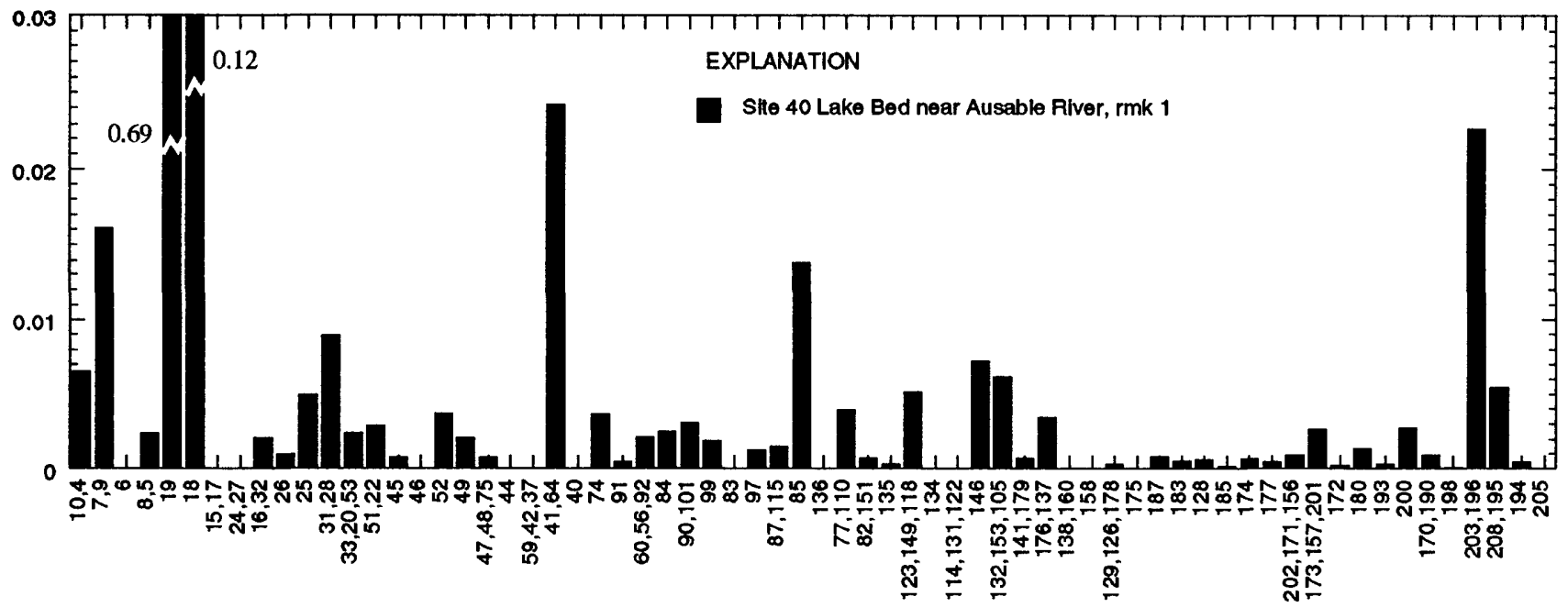

POLYCHLORINATED BIPHENYL CONGENER NUMBER

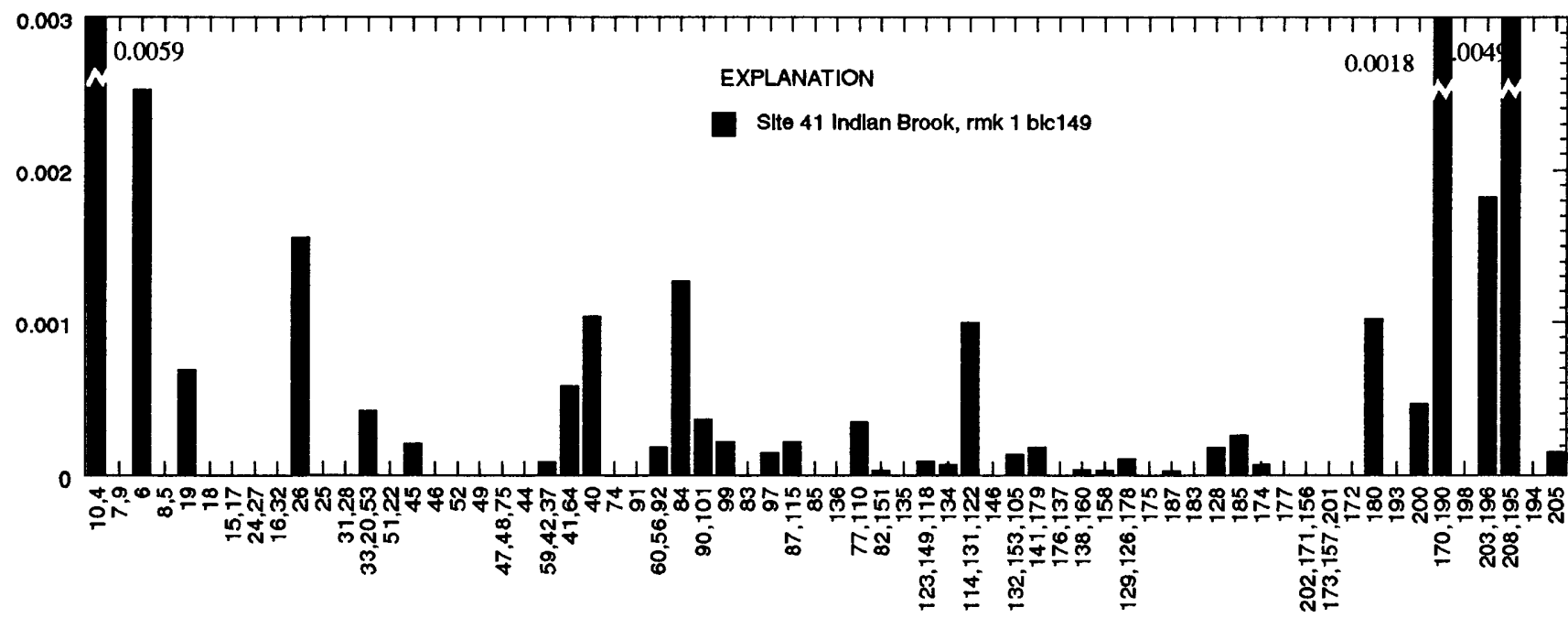

POLYCHLORINATED BIPHENYL CONGENER NUMBER

表

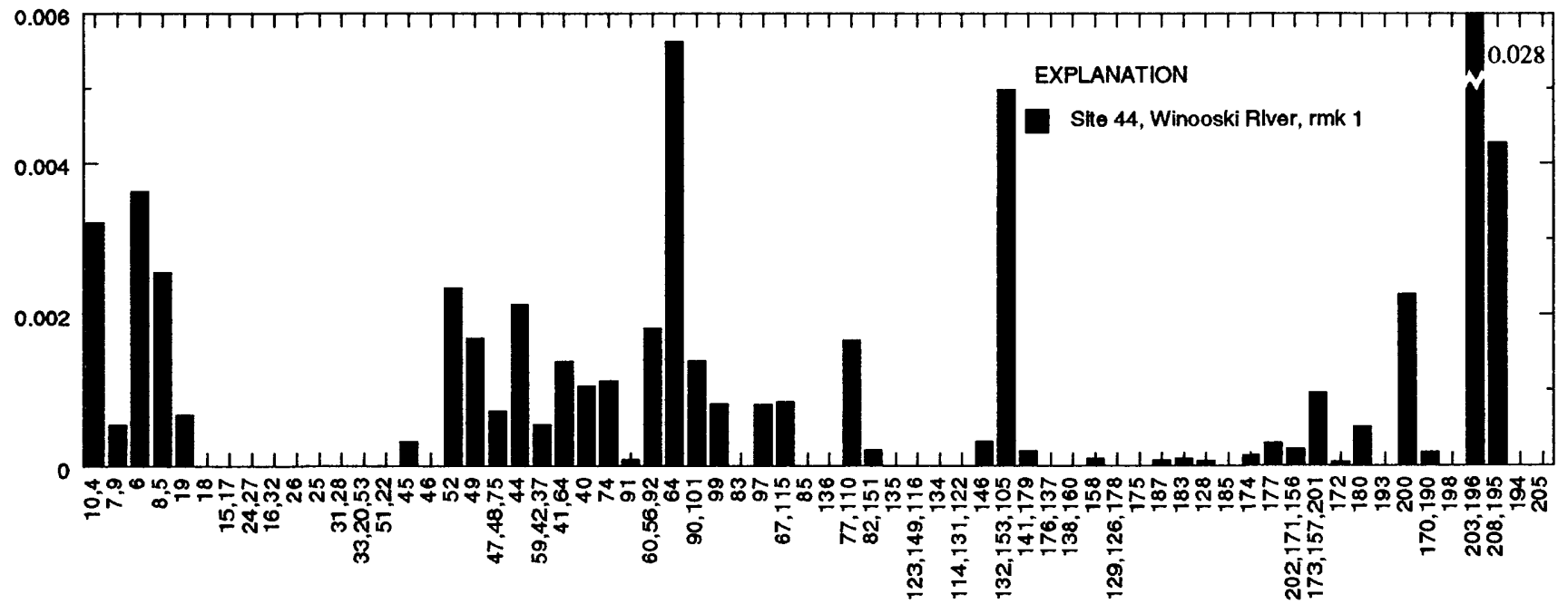

POLYCHLORINATED BIPHENYL CONGENER NUMBER

Figure 2. Histograms of polychlorinated biphenyl congeners (PCBs) from stream- and lake-bed samples of Lake Champlain. Site numbers are listed in figure 1 and site descriptions in table 1. Congener identifications are listed by International Union of Practical and Applied Chemistry number in table 7. Concentration units apply only if height of peak is caused by PCB congeners as described in text; rmk refers to Design remark in table 9.--Continued 


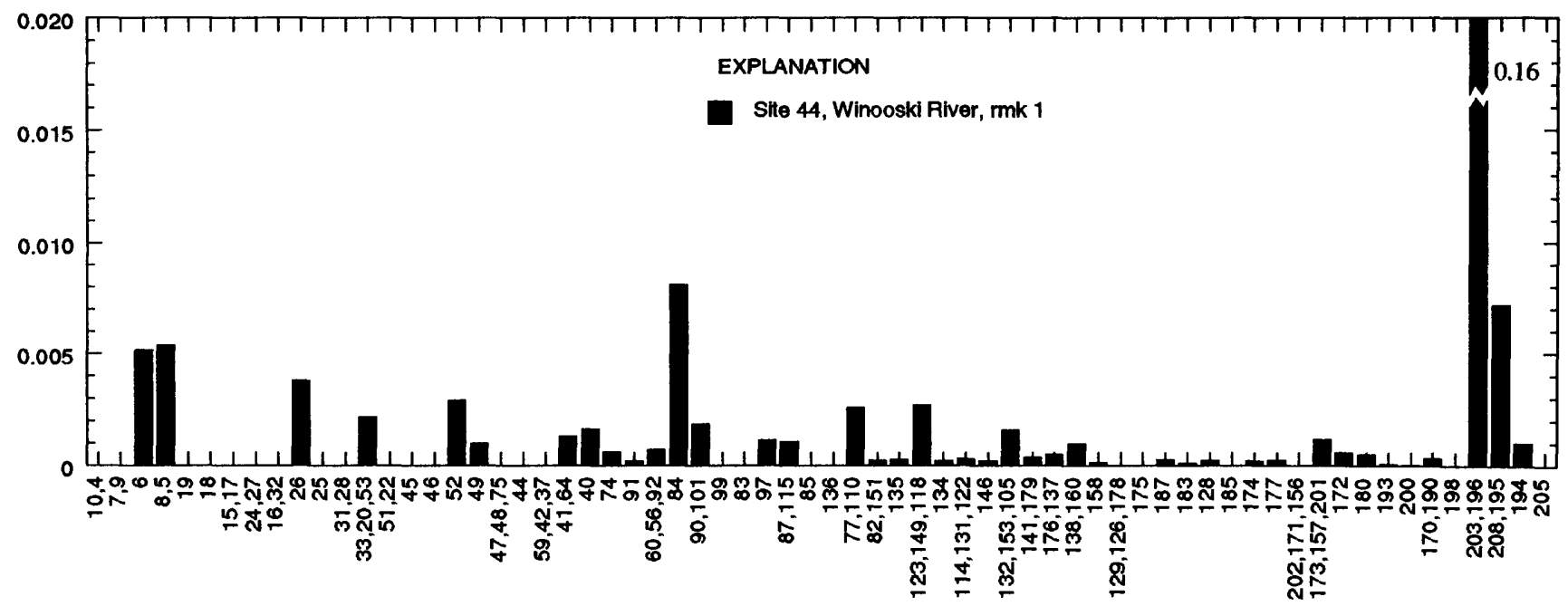

POLYCHLORINATED BIPHENYL CONGENER NUMBER

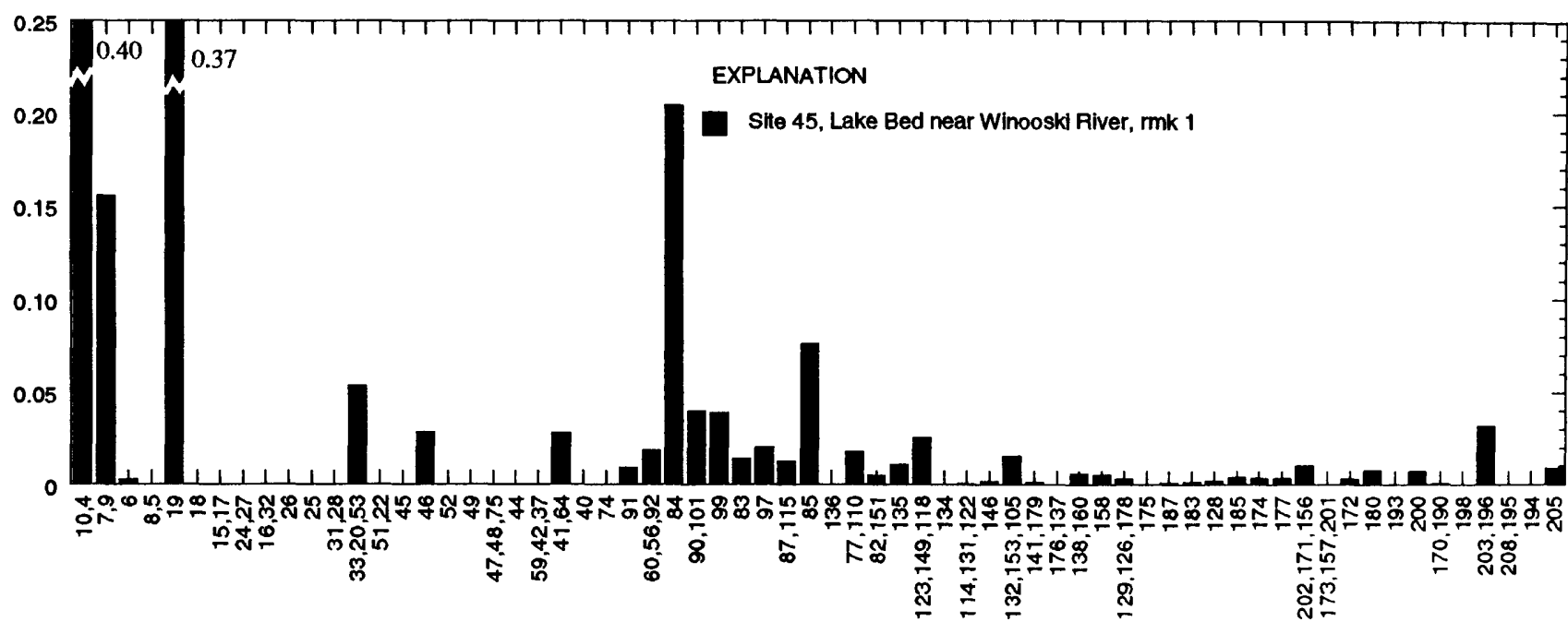

POLYCHLORINATED BIPHENYL CONGENER NUMBER

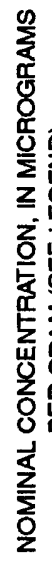

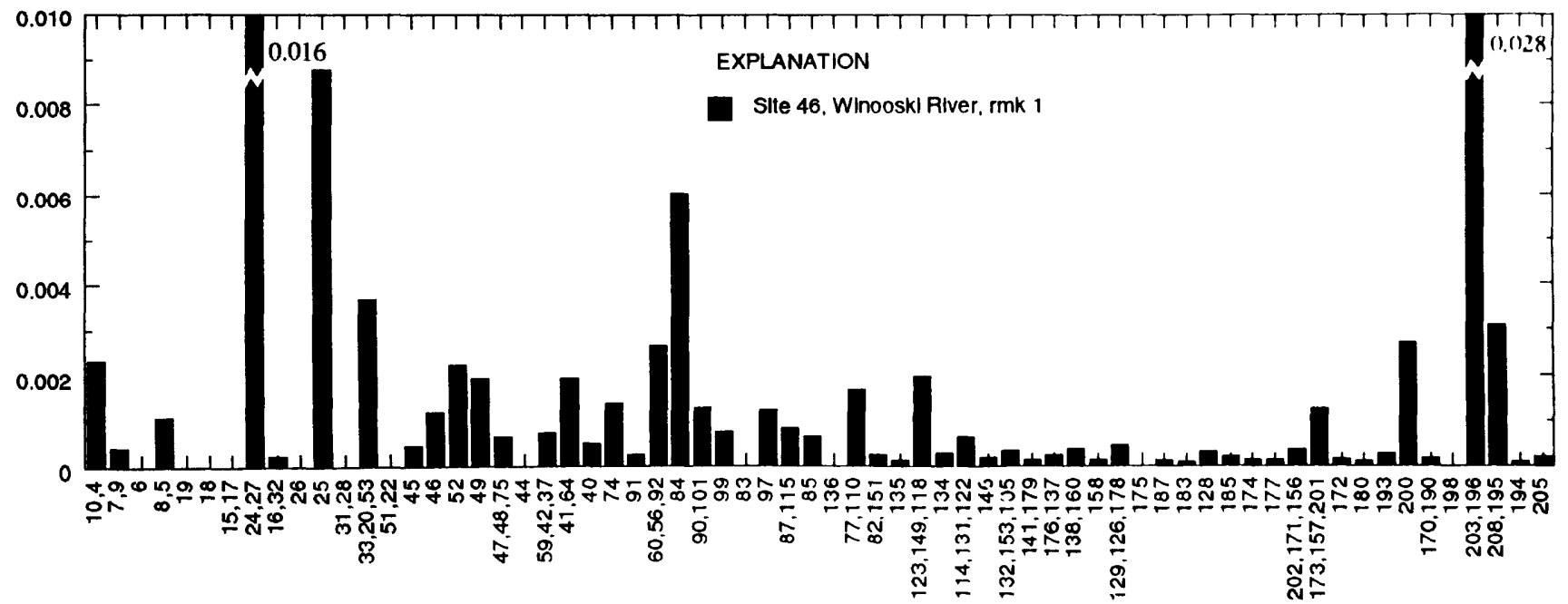

POLYCHLORINATED BIPHENYL CONGENER NUMBER

Figure 2. Histograms of polychlorinated biphenyl congeners (PCBs) from stream- and lake-bed samples of Lake Champlain. Site numbers are listed in figure 1 and site descriptions in table 1. Congener identifications are listed hy International Union of Practical and Applied Chemistry number in table 7. Concentration units apply only if height of peak is caused by I'CB congeners as described in text; rmk refers to Design remark in table 9.--Continued 


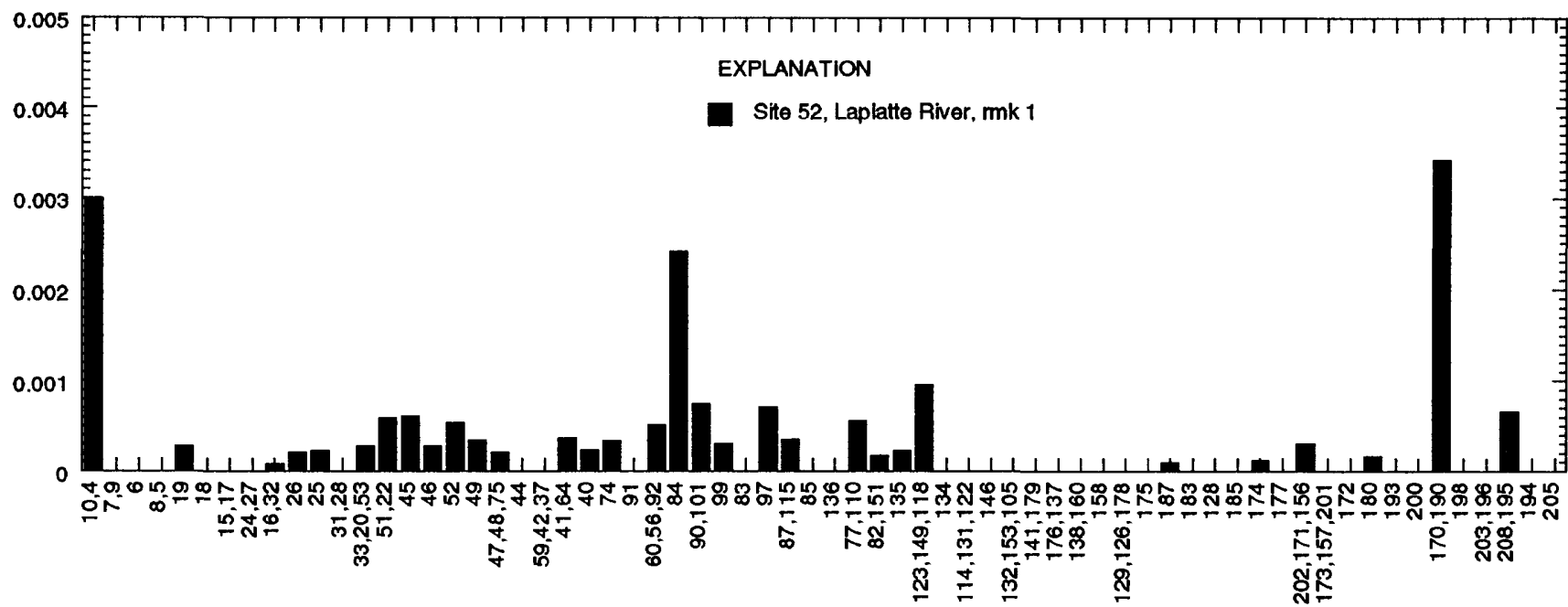

POLYCHLORINATED BIPHENYL CONGENER NUMBER

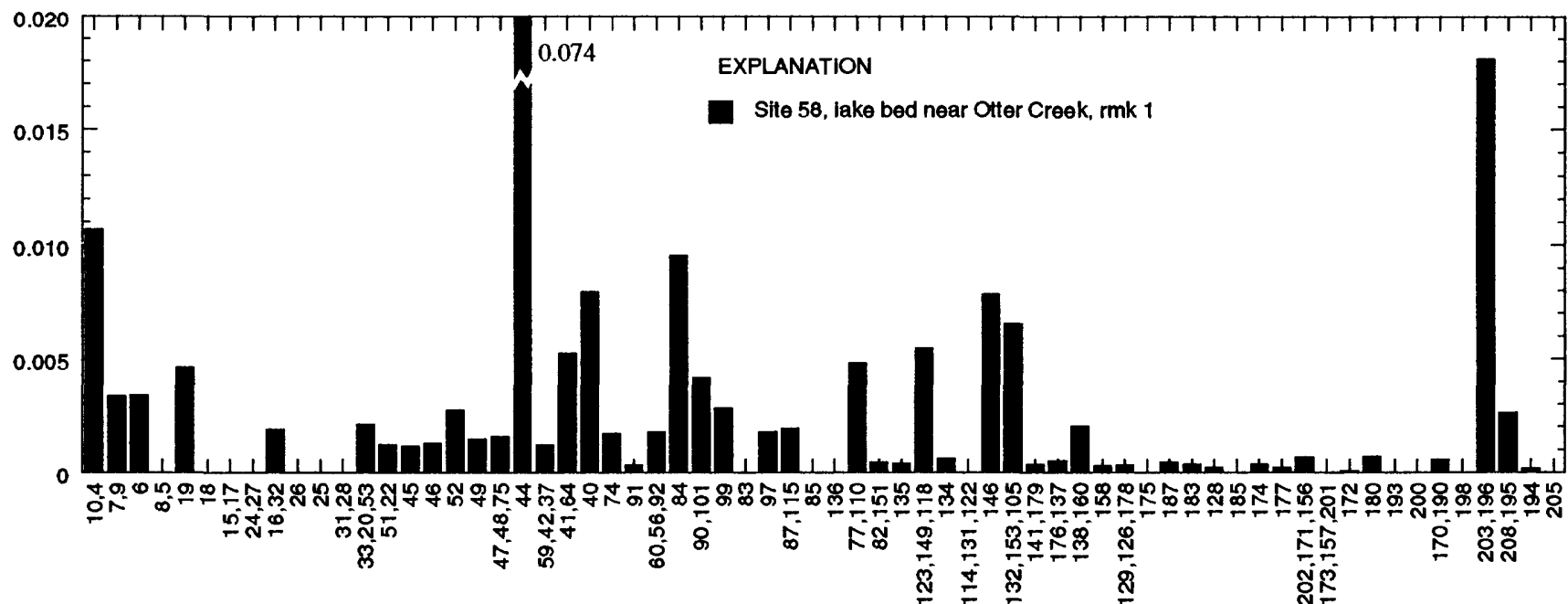

POLYCHLORINATED BIPHENYL CONGENER NUMBER

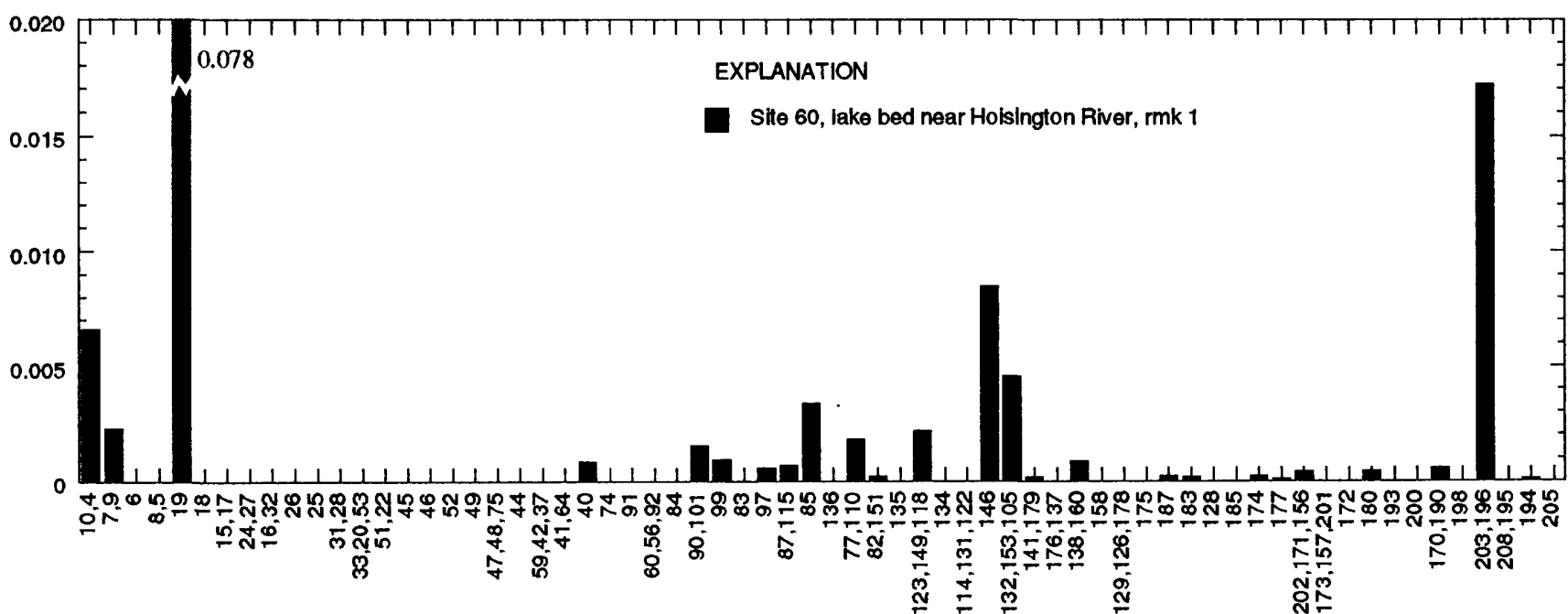

POLYCHLORINATED BIPHENYL CONGENER NUMBER

Figure 2. Histograms of polychlorinated biphenyl congeners (PCBs) from stream- and lake-bed samples of Lake Champlain. Site numbers are listed in figure 1 and site descriptions in table 1. Congener identifications are listed by International Union of Practical and Applied Chemistry number in table 7. Concentration units apply only if height of peak is caused by PCB congeners as described in text; rmk refers to Design remark in table 9.--Continued 


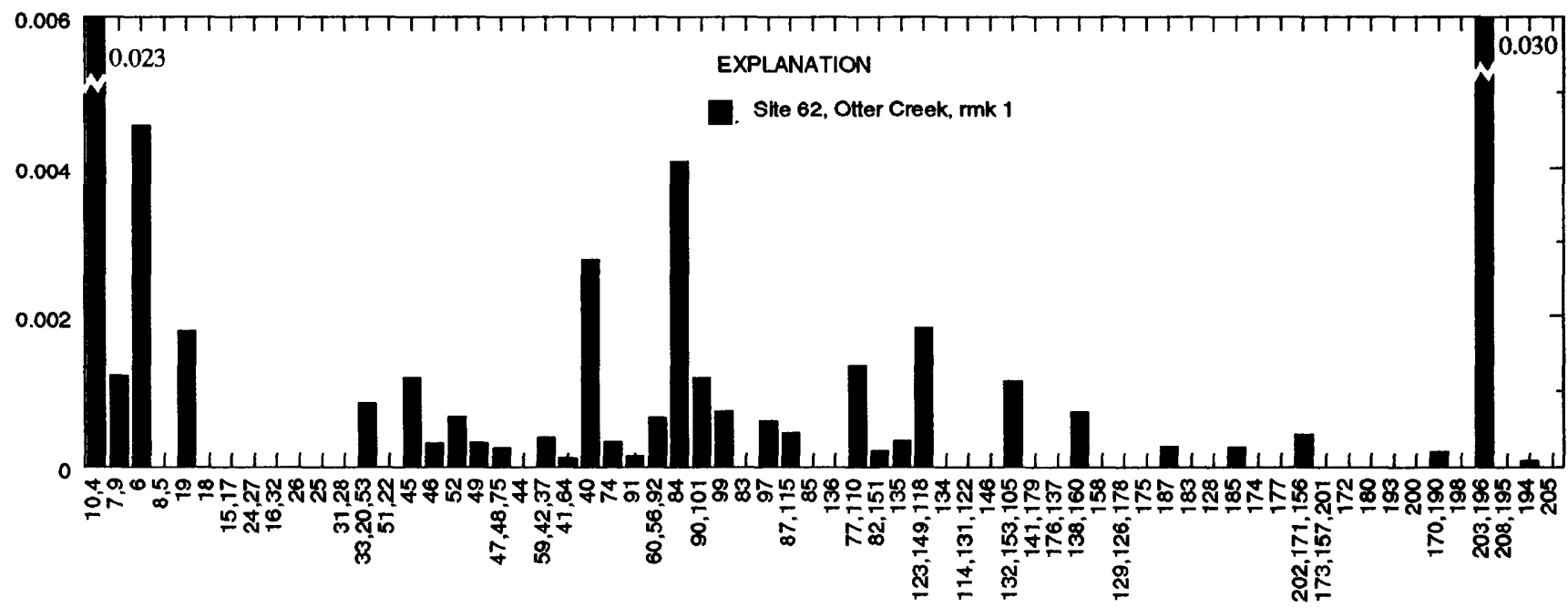

POLYCHLORINATED BIPHENYL CONGENER NUMBER

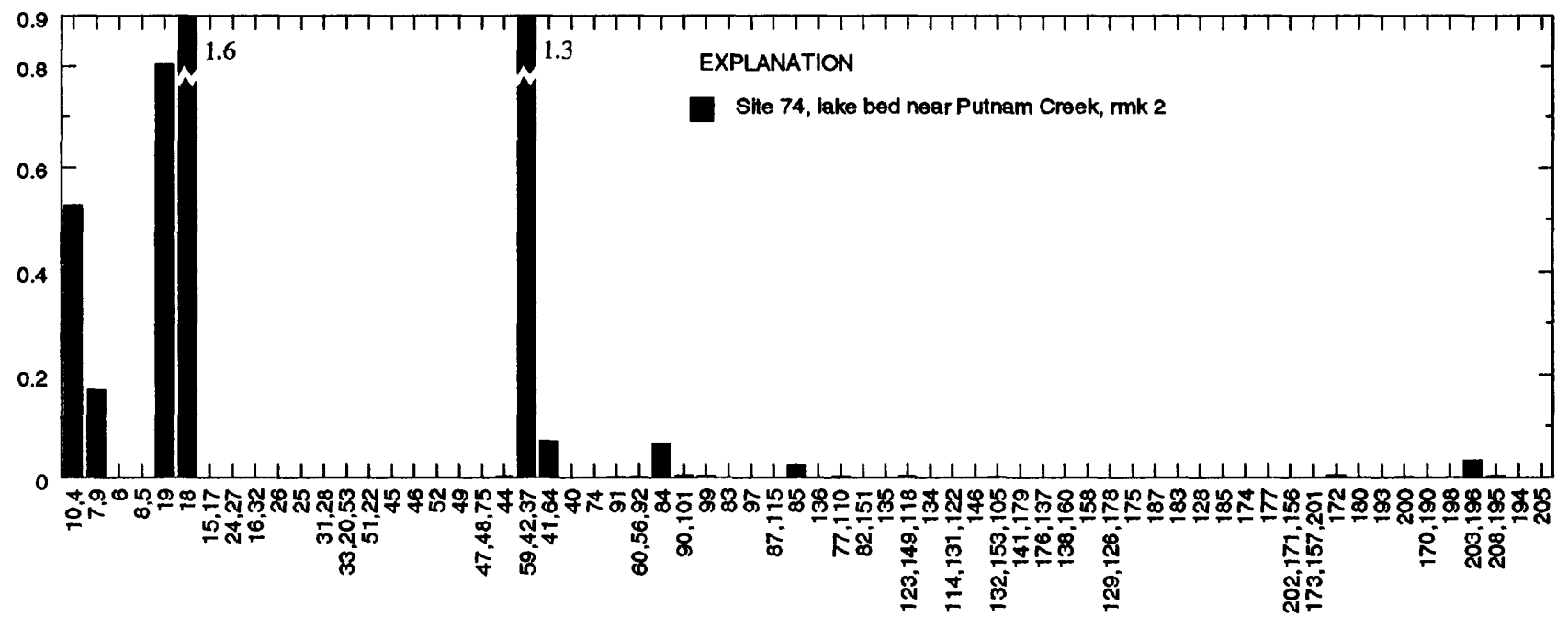

POLYCHLORINATED BIPHENYL CONGENER NUMBER
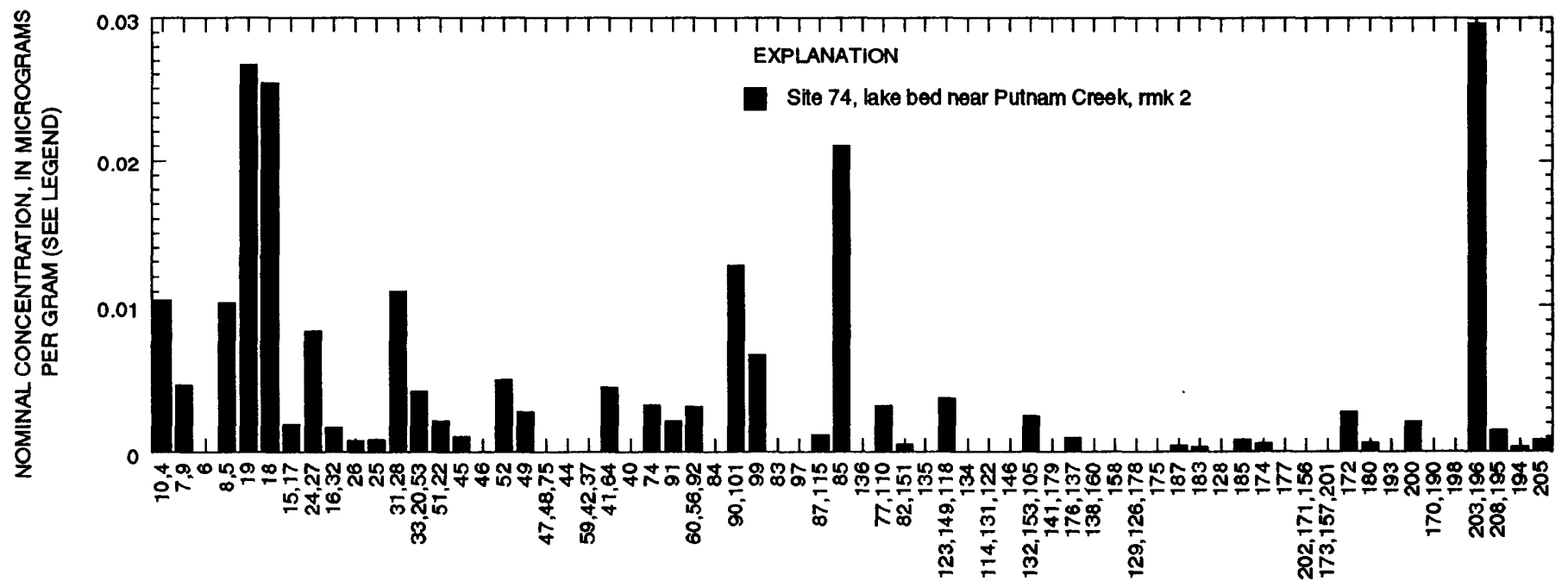

POLYCHLORINATED BIPHENYL CONGENER NUMBER

Figure 2. Histograms of polychlorinated biphenyl congeners (PCBs) from stream- and lake-bed samples of Lake Champlain. Site numbers are listed in figure 1 and site descriptions in table 1. Congener identifications are listed by International Union of Practical and Applied Chemistry number in table 7. Concentration units apply only if height of peak is caused by PCB congeners as described in text; rmk refers to Design remark in table 9.--Continued 


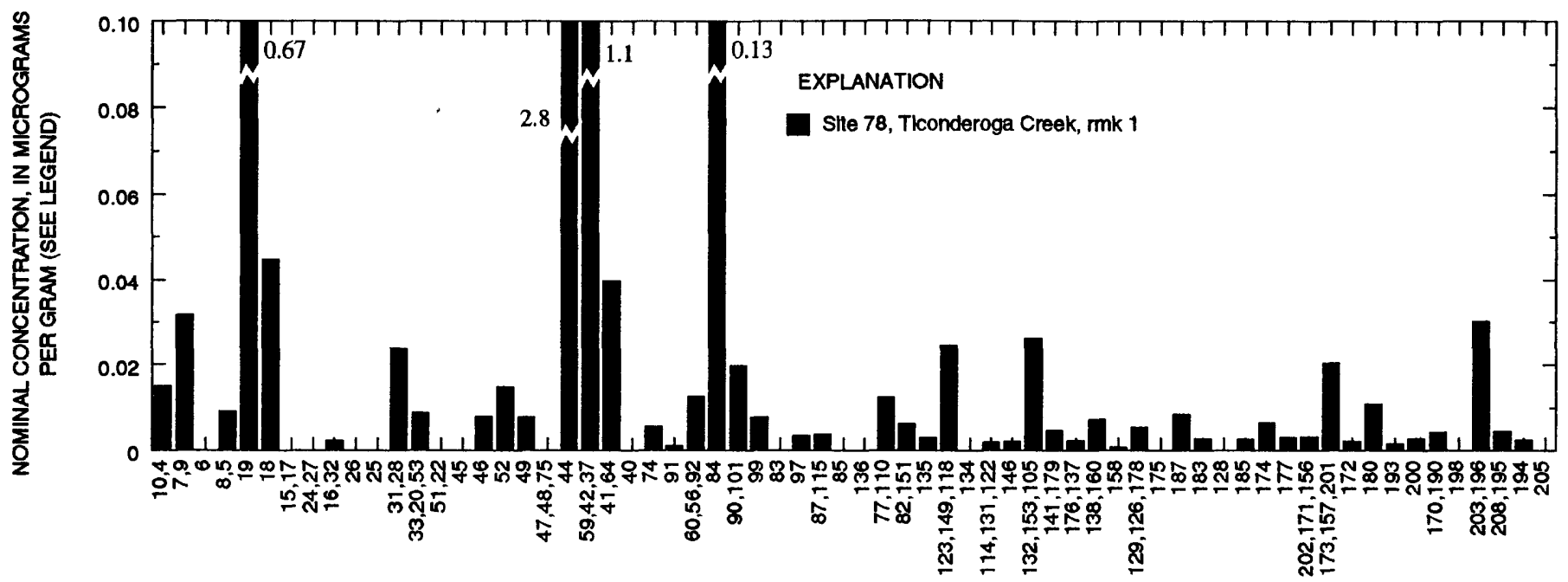

POLYCHLORINATED BIPHENYL CONGENER NUMBER
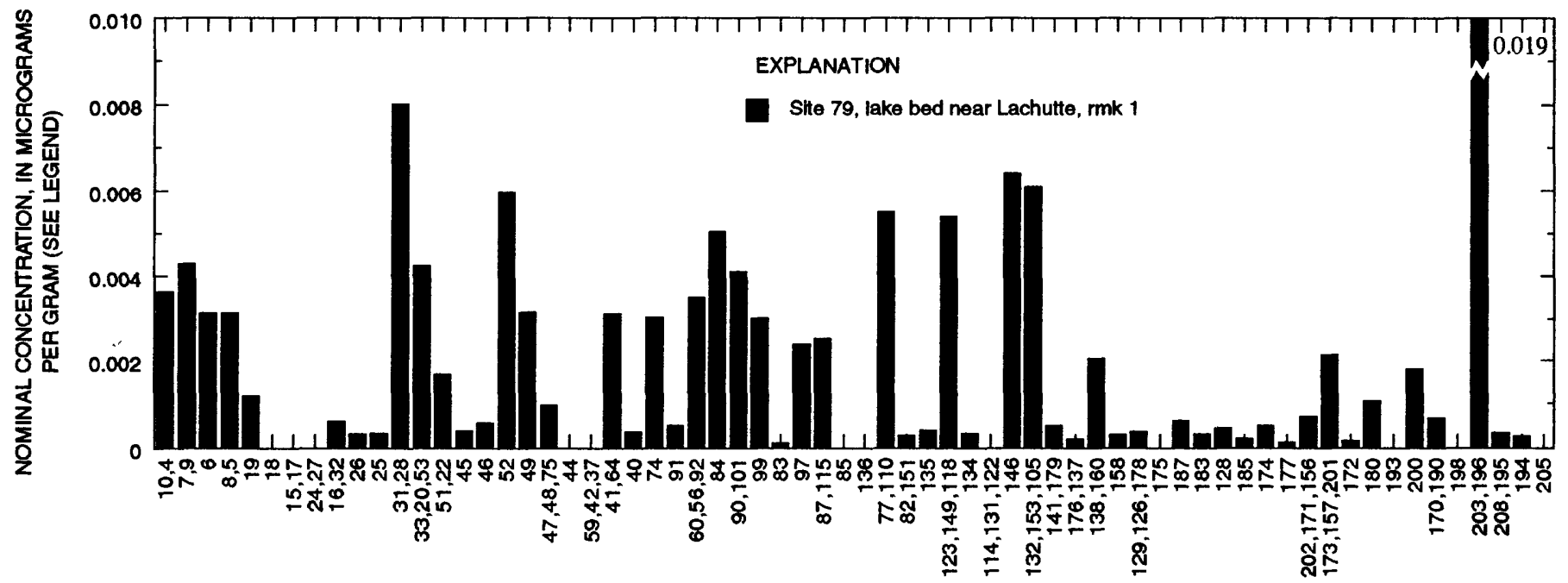

POLYCHLORINATED BIPHENYL CONGENER NUMBER
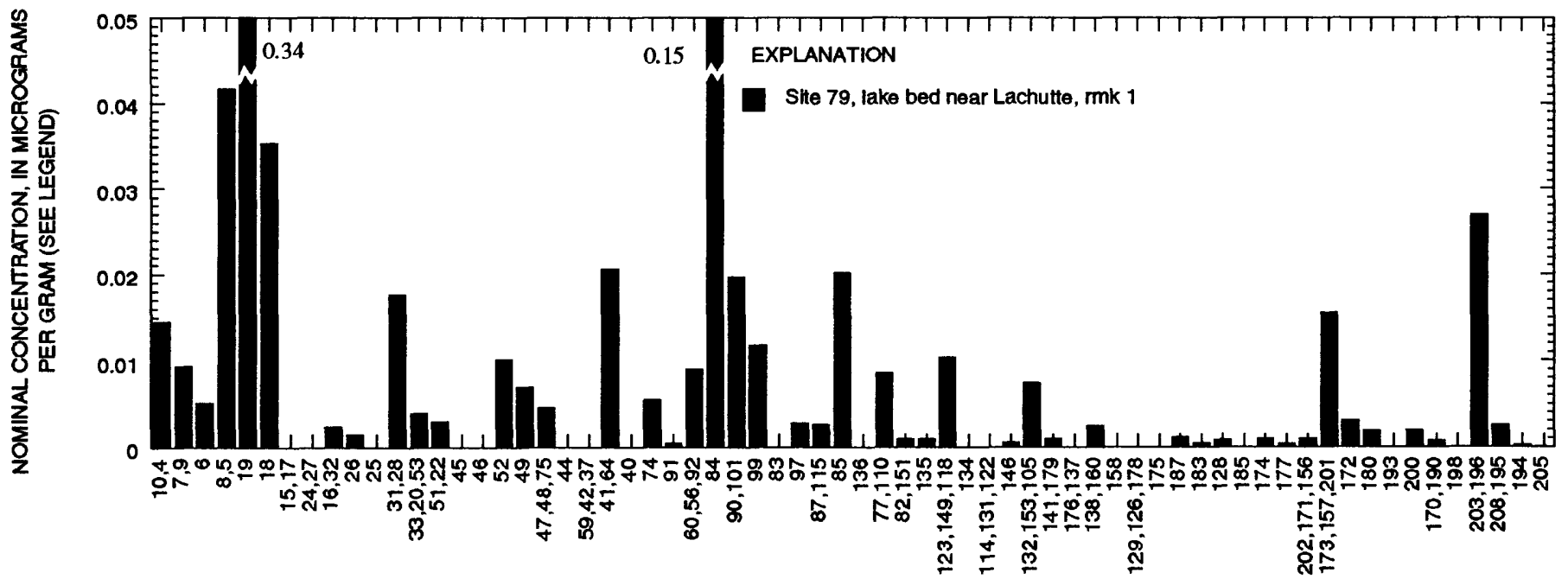

POLYCHLORINATED BIPHENYL CONGENER NUMBER

Figure 2. Histograms of polychlorinated biphenyl congeners (PCBs) from stream-and lake-bed samples of Lake Champlain. Site numbers are listed in figure 1 and site descriptions in table 1. Congener identifications are listed by International Union of Practical and Applied Chemistry number in table 7. Concentration units apply only if height of peak is caused by PCB congeners as described in text; rmk refers to Design remark in table 9.--Continued 


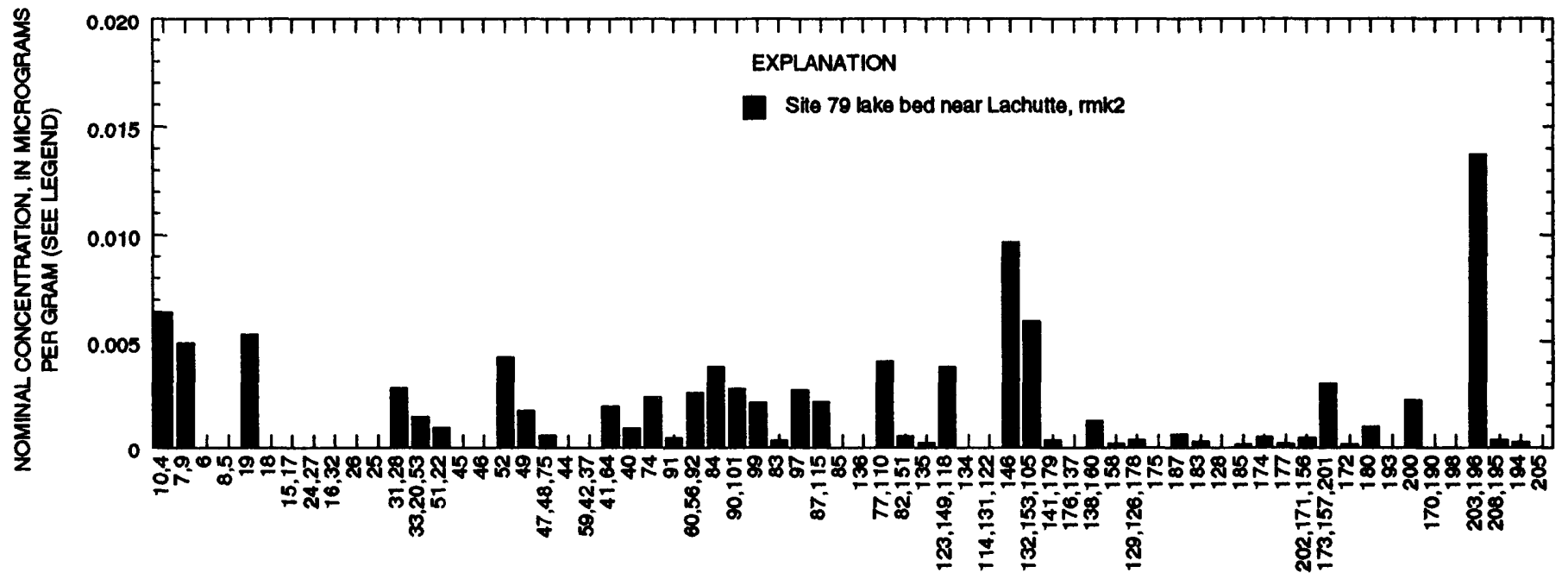

POLYCHLORINATED BIPHENYL CONGENER NUMBER

Figure 2. Histograms of polychlorinated biphenyl congeners (PCBs) from stream- and lake-bed samples of Lake Champlain. Site numbers are listed in figure 1 and site descriptions in table 1. Congener identifications are listed by International Union of Practical and Applied Chemistry number in table 7. Concentration units apply only if height of peak is caused by PCB congeners as described in text; rmk refers to Design remark in table 9.--Continued 


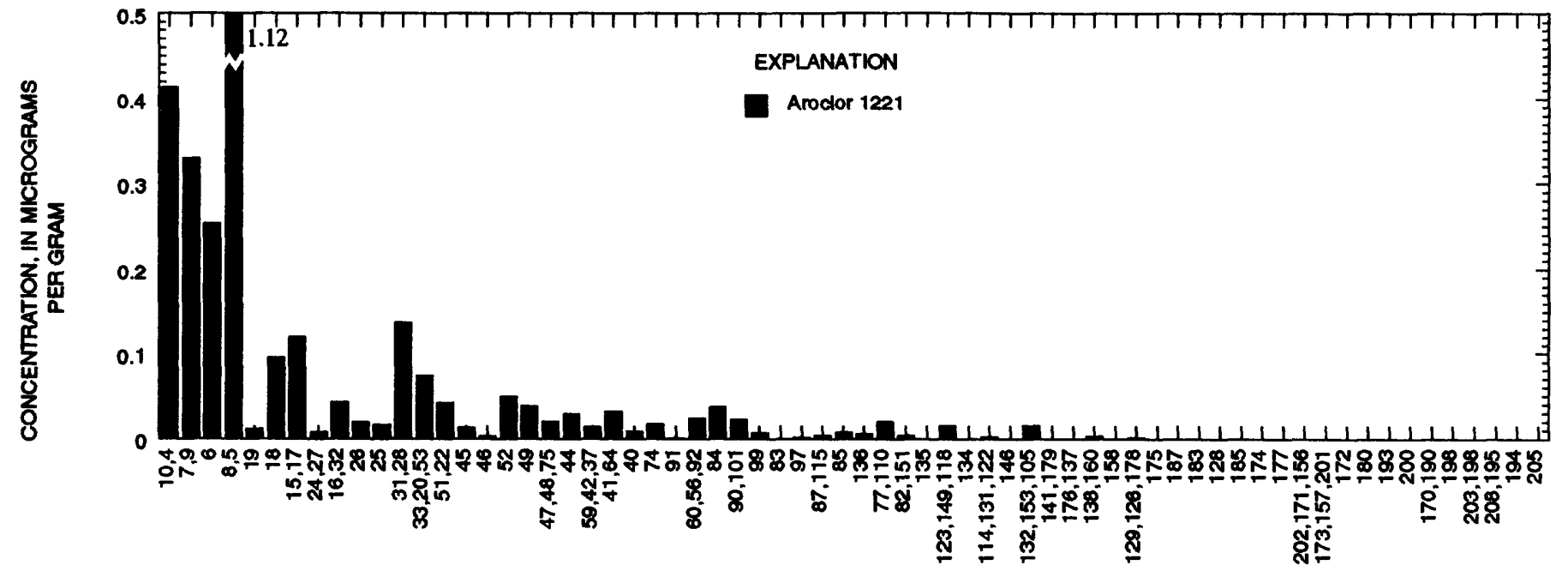

POLYCHLORINATED BIPHENYL CONGENER NUMBER

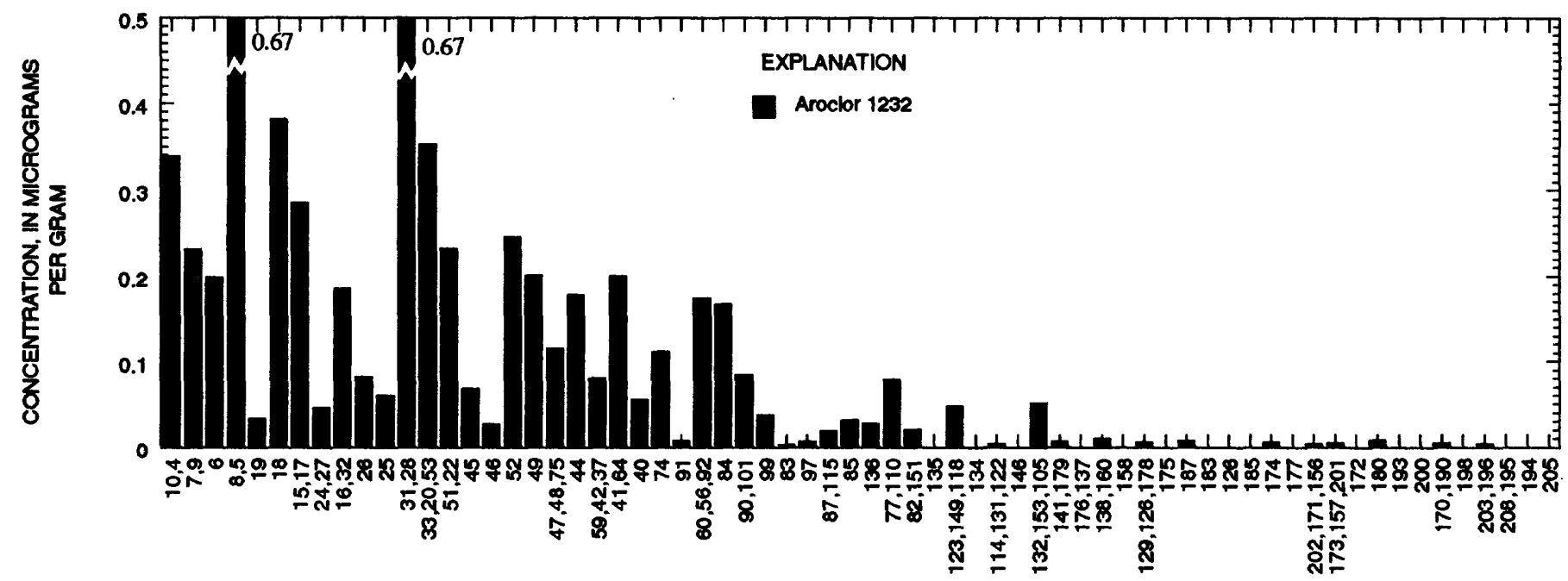

POLYCHLORINATED BIPHENYL CONGENER NUMBER

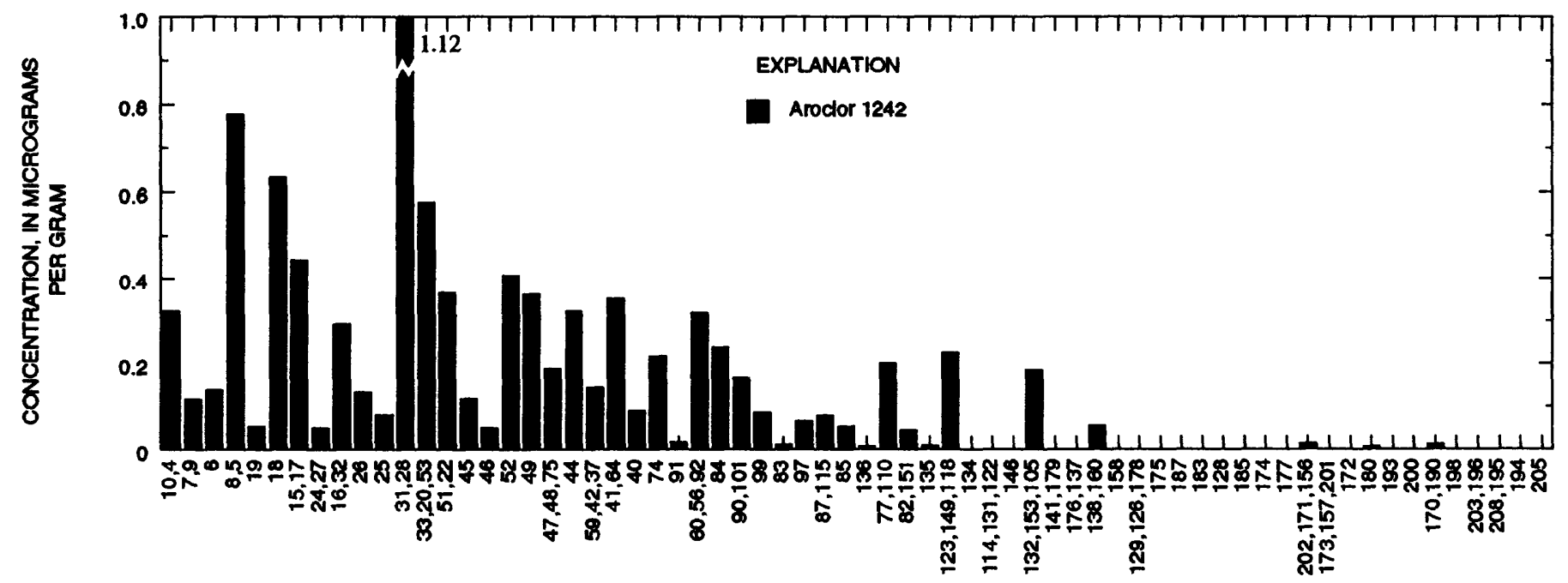

POLYCHLORINATED BIPHENYL CONGENER NUMBER

Figure 3. Histograms of polychlorinated biphenyl congeners in Aroclor mixtures. Congener identifications are by International Union of Practical and Applied Chemistry number, as listed in table 7. 


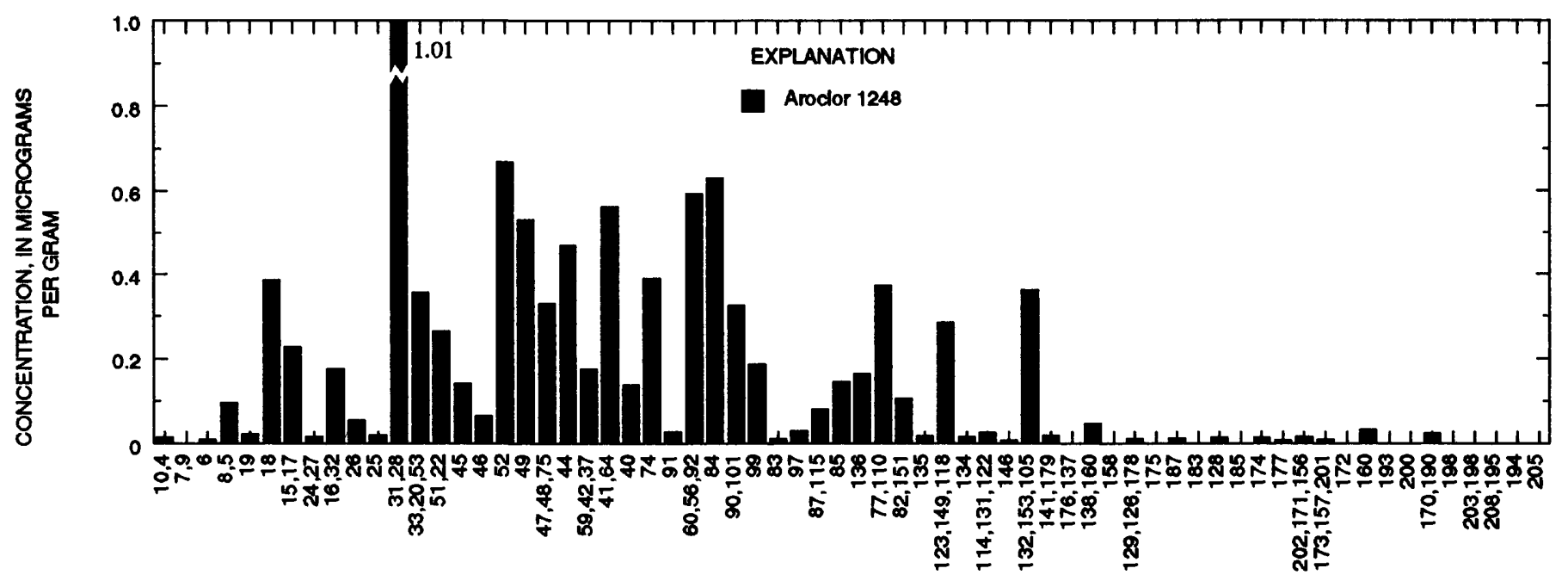

POLYCHLORINATED BIPHENYL CONGENER NUMBER

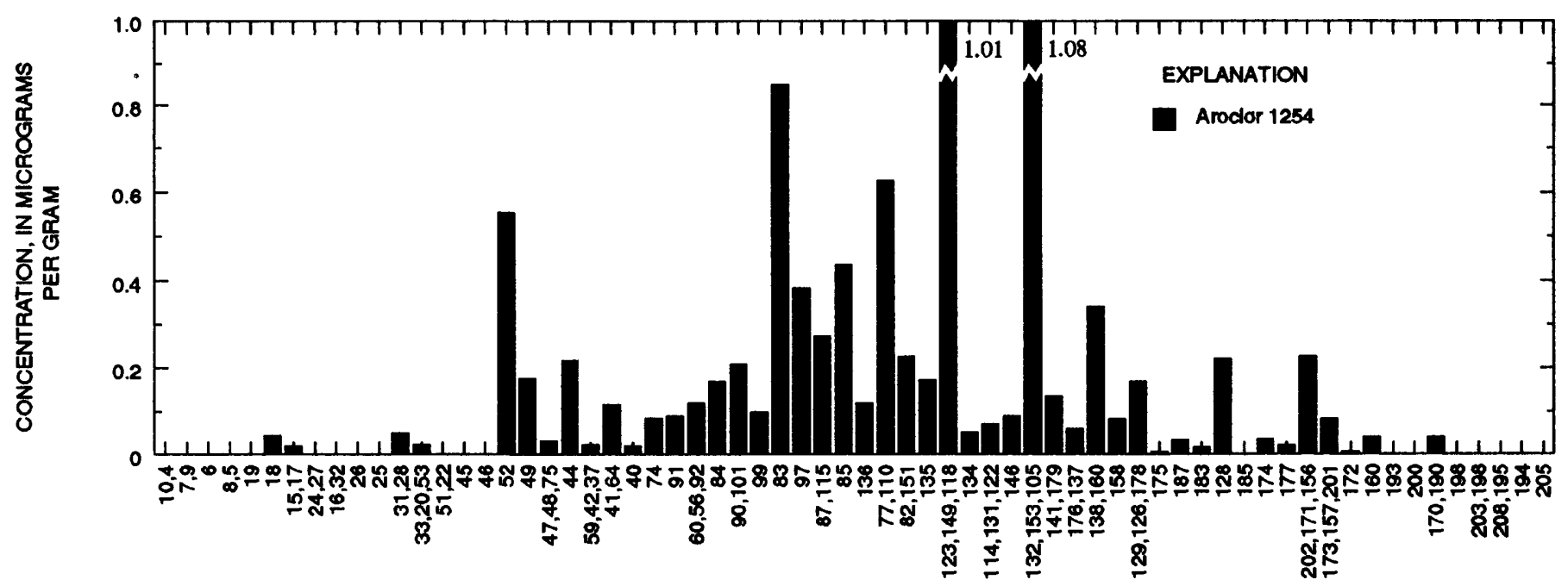

POLYCHLORINATED BIPHENYL CONGENER NUMBER

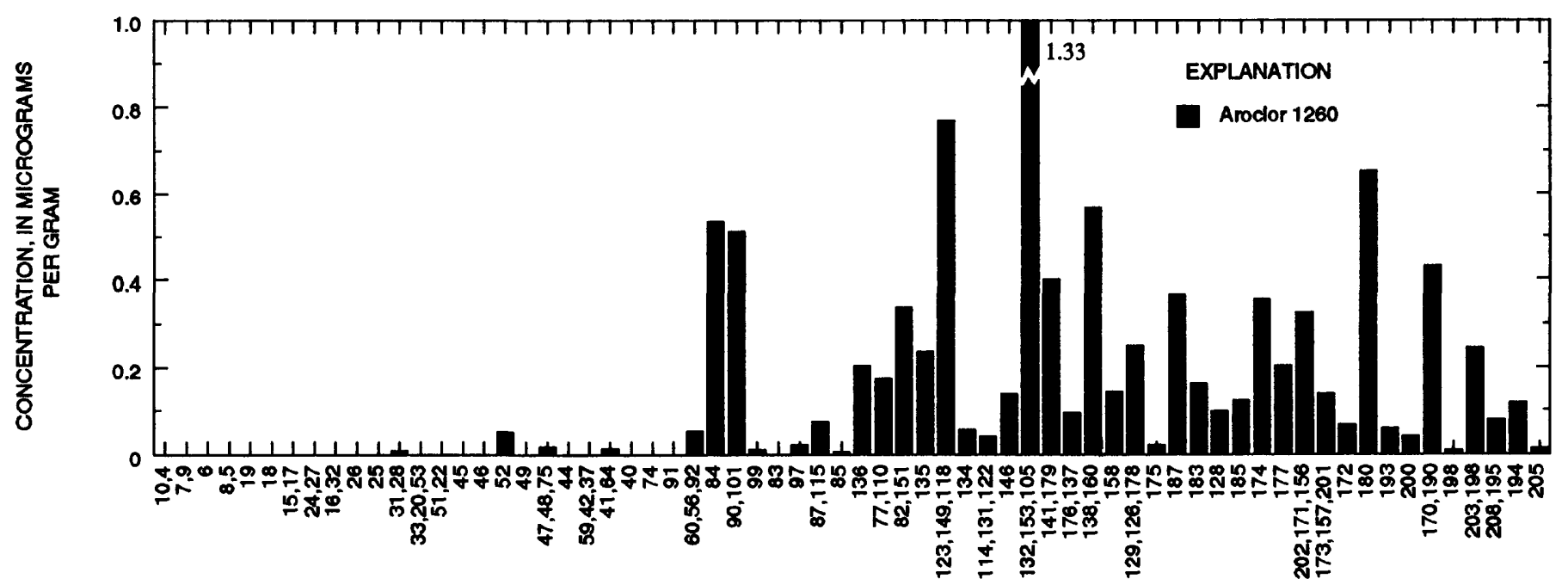

POLYCHLORINATED BIPHENYL CONGENER NUMBER

Figure 3. Histograms of polychlorinated biphenyl congeners in Aroclor mixtures. Congener identifications are by International Union of Practical and Applied Chemistry number, as listed in table 7.--Continued 